\title{
Measurement of neutron star parameters: a review of methods for low-mass X-ray binaries
}

\author{
Sudip Bhattacharyya \\ Department of Astronomy and Astrophysics, Tata Institute of Fundamental Research, Mumbai 400005, India
}

\begin{abstract}
Measurement of at least three independent parameters, for example, mass, radius and spin frequency, of a neutron star is probably the only way to understand the nature of its supranuclear core matter. Such a measurement is extremely 'difficult because of various systematic uncertainties. The lack of knowledge of several system parameter values gives rise to such systematics. Low mass X-ray binaries, which contain neutron stars, provide a number of methods to constrain the stellar parameters. Joint application of these methods has a great potential to significantly reduce the systematic uncertainties, and hence to measure three independent neutron star parameters accurately. Here we review the methods based on (1) thermonuclear X-ray bursts; (2) accretion-powered millisecond-period pulsations; (3) kilohertz quasi-periodic oscillations; (4) broad relativistic iron lines; (5) quiescent emissions; and (6) binary orbital motions.
\end{abstract}

'Key words: dense matter, equation of state, relativity, stars: fundamental parameters, stars: neutron, X-rays: binaries

\section{Contents}

1 Introduction

2 Why This Review and Why Now?

3 Methods

3.1 Thermonuclear X-ray Bursts

3.1.1 What is a Thermonuclear X-ray Burst

3.1.2 Why are Thermonuclear X-ray Bursts Useful?

3.1.3 Continuum Spectrum Method

3.1.4 Spectral Line Method

3.1.5 Photospheric Radius Expansion Burst Method

3.1.6 Burst Oscillation Method

3.1.7 Millihertz Quasi-periodic Oscillation Method

3.1.8 Joint Application of the Methods based on

Thermonuclear X-ray Bursts

3.2 Accretion-powered Millisecond Period Pulsations

3.2.1 What is an Accretion-powered Millisecond Pulsar?

3.2.2 Accretion-powered Millisecond Pulsation Method 3.3 Kilohertz Quasi-periodic Oscillations

3.3.1 What are Kilohertz Quasi-periodic Oscillations?

3.3.2 Kilohertz Quasi-periodic Oscillation Method

3.4 Broad Relativistic Iron Lines

3.4.1 What is a Broad Relativistic Iron Line

3.4.2 Broad Relativistic Iron Line Method

3.5 Quiescent Emissions

3.5.1 What is Quiescent Emission?

3.5.2 Quiescent Emission Method

3.6 Mass Measurement: Binary Orbital Motion Method 4 Why are Low-mass X-ray Binaries Useful? 5 Summary and Future Prospects

Email address: sudip@tifr.res.in (Sudip Bhattacharyya)

\section{Introduction}

The nature of matter at ultra-high density, i.e., $5-10$ times higher than the nuclear density, but at a relatively low temperature (e.g., $\sim 10^{8} \mathrm{~K}$ ) cannot be probed by heavy-nuclei collision experiments or with observations of the early universe (Özel and Psaltis (2009); van Kerkwijk (2004); Blaschke et al. (2008); Lattimer and Prakash (2007) and references therein). This matter exists in the neutron star cores, and hence these stars provide natural laboratories to study the supranuclear degenerate matter. The study of this dense matter is not only important from the point of view of the particle physics, but also can be useful to understand the core collapse of massive stars, the supernova phenomenon, the existence and properties of neutron stars, etc. (e.g., van Kerkwijk (2004)).

But how to observe and understand the deep interior of a neutron star? Based on the assumed microscopic properties of the core matter, many equation of state (EoS) models have been proposed in the literature. These models connect the pressure $(p)$ with the total mass-energy density $(\epsilon)$ in a degenerate condition. Therefore, for a nonspinning neutron star and for a given EoS model, the stellar global structure can be calculated by solving the Tolman-Oppenheimer-Volkoff (TOV) equation:

$$
\frac{\mathrm{d} p(r)}{\mathrm{d} r}=-\frac{G}{c^{2}} \frac{[p(r)+\epsilon(r)]\left[m(r)+4 \pi r^{3} p(r) / c^{2}\right]}{r\left(r-2 G m(r) / c^{2}\right)},
$$

where, $m(r)=4 \pi \int_{0}^{r} \mathrm{~d} r^{\prime} r^{\prime 2} \epsilon\left(r^{\prime}\right)$ is the gravitational mass (hereafter, mass) inside a sphere of radius $r$. For a rapidly spinning neutron star, Cook et al. (1994) and other authors have suggested ways to compute the stellar structure (see the dashed curves of Figure 1; see also, Datta et 
al. (1998); Thampan et al. (1999); Bhattacharyya et al. (2000, 2001a,b)). The Figure 1 shows that for a given EoS model and a known stellar spin frequency the stable structures of neutron stars trace a single mass $(M)$-radius $(R)$ curve. However, such curves for different EoS models can intersect each other (Figure 1). Therefore, it is required to accurately measure at least three independent global parameters of the same neutron star in order to constrain the EoS models, and hence to understand the supranuclear core matter. This is also true for the EoS models of strange matter (made of $u, d$ and $s$ quarks; Witten (1984); Farhi and Jaffe (1984); Bombaci (1997); Bombaci et al. (2000); Bhattacharyya et al. (2001c); Bagchi et al. (2006)). However, some neutron star EoS models can be ruled out only by mass measurement. Here is how. A stable neutron star's mass cannot be greater than a maximum value $\left(M_{\max }\right.$; Lattimer and Prakash (2007)) for a given EoS model and spin frequency. Therefore, a large measured mass value can rule out some "softer" EoS models (for example, "NS1" in Figure 1), that have lower $M_{\max }$ than the "harder" models (for example, "NS4" in Figure 1; see also Lattimer and Prakash (2007)).

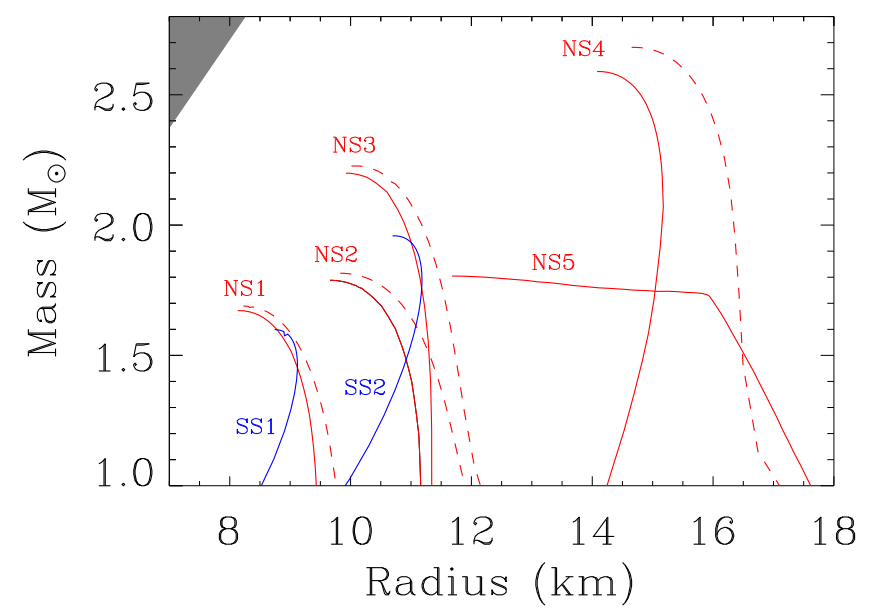

Figure 1: This figure shows the mass-radius $(M-R)$ space of neutron stars and the curves corresponding to a few representative EoS models $(\S 1)$. The solid curves are for nonspinning stars, while the dashed curves (corresponding to some of the solid curves) are for a stellar spin frequency of $600 \mathrm{~Hz}$. The red curves are for the usual neutron star EoS models: NS1 (A18 model of Akmal et al. (1998)); NS2 (Baldo et al. , 1997); NS3 (A18+ $\delta v+$ UIX model of Akmal et al. (1998)); NS4 (Sahu et al. , 1993); and NS5 (Pandharipande and Smith , 1974); while the blue curves are for the strange matter EoS models: SS1 (bag constant $=90 \mathrm{MeV} \mathrm{fm}^{-3}$; Farhi and Jaffe (1984)) and SS2 (bag constant $=60 \mathrm{MeV} \mathrm{fm}^{-3}$; Farhi and Jaffe (1984); see also $\S 1$ ). The grey region indicates the $M-R$ space of black holes. Here we have shown the $M>1 M_{\odot}$ region, because many neutron stars in double neutron star binaries have the mass around $1.35 M_{\odot}$ (Thorsett and Chakrabarty , 1999), and therefore the accreting neutron stars in low-mass X-ray binaries should have even larger mass values (see $\S 4$ ). This figure suggests that the measurement of mass, radius and spin frequency of the same neutron star is required to constrain the EoS models.
The above discussion give the motivation for an accurate measurement of the neutron star parameters. In this review, we discuss the measurement methods relevant for neutron stars in low-mass X-ray binary (LMXB) systems (Bhattacharya and van den Heuvel, 1991). An LMXB is an old binary stellar system (typical age $\sim 10^{9}$ years) with a low-mass companion star $\left(\leq 1 M_{\odot}\right)$ and a compact star rotating around each other. The companion star may be a main sequence star, an evolved star or a white dwarf, while the compact star may be a neutron star or a black hole. In such a system, the companion star fills its Roche lobe, i.e., the critical equipotential surface (see Bhattacharya and van den Heuvel (1991)), and matter from it flows towards the compact star (neutron star in our case). This matter cannot move radially towards the neutron star, as its initial angular momentum is large. Therefore, the accreted matter slowly gets rid of the angular momentum (Shakura and Sunyaev , 1973), follows spiral paths, and hence forms an accretion disk. In neutron star LMXBs, the accreted matter eventually hits the stellar surface, and generates electromagnetic radiation. The accretion disk also emits such radiation by viscous dissipation, and both these emissions are powered by the gravitational potential energy release. The inner part of the accretion disk, and the neutron star surface emit X-rays, as their typical temperature is $\sim 10^{7} \mathrm{~K}$. These $\mathrm{X}$-rays are reprocessed by the outer part of the accretion disk, which emits in optical wavelengths. Moreover, the companion star may be irradiated by these X-rays. The neutron stars in LMXBs are expected to be spun up, due to the angular momentum transferred via accretion. This is consistent with the observed stellar spin frequencies (typically $\sim 300-600 \mathrm{~Hz}$; see $\S 3.1 .6 ; \oint 3.2 .2)$. The magnetic fields $\left(\approx 10^{7}-10^{9} \mathrm{G}\right)$ of these neutron stars are typically much lower than that of the isolated neutron stars and the stars in high-mass Xray binary (HMXB) systems (Bhattacharya and van den Heuvel , 1991; Ibragimov and Poutanen , 2009; Psaltis and Chakrabarty , 1999). Several groups have worked on this problem of stellar magnetic field evolution (Srinivasan et al. , 1990; Jahan Miri and Bhattacharya , 1995; Konar et al. , 1995; Choudhuri and Konar , 2002; Konar and Choudhuri , 2004). Note that the low magnetic field allows the accretion disk to extend up to very close to the neutron star. This, in combination with the fact that X-rays can be detected both from the star and the inner part of the disk for many LMXBs, provides an excellent opportunity to measure the neutron star parameters. Moreover, the signatures of binary orbital motion detected from the optical observation of the accretion disk and the companion star can be useful to measure the neutron star mass.

LMXBs are observed mostly in the Galactic plane and bulge, and in the globular clusters. Some of them have also been discovered from the nearby galaxies. According to the catalogue of Liu et al. (2007), 187 LMXBs (containing either a neutron star or a black hole) were known from our galaxy, "Large Magellanic Cloud" and "Small Magellanic Cloud" up to that time. As indicated from 
thermonuclear bursts, mass function, etc., most of these LMXBs contain a neutron star, rather than a black hole. LMXBs emit primarily in X-ray wavelengths, and the optical to X-ray luminosity ratio is normally less than 0.1 for them (see Bhattacharya and van den Heuvel (1991)). The central X-ray emission from these sources cannot be spatially resolved using the current X-ray imaging instruments, because the distances of even our Galactic LMXBs are very large (typically $>1 \mathrm{kpc}$ ). Therefore, one needs to rely on the spectral and timing properties in order to study these sources in X-rays.

X-rays from space cannot reach the surface of the Earth. Therefore, the X-ray detectors and telescopes are sent above the atmosphere, typically by satellites. The current primary X-ray space missions are (1) NASA's Rossi X-ray Timing Explorer (RXTE); (2) ESA's XMM-Newton; (3) NASA's Chandra; and (4) JAXA's Suzaku. They primarily operate in the $\approx 1-10 \mathrm{keV}$ range (i.e., soft $\mathrm{X}$ rays), and contain a variety of instruments. The primary instrument of $R X T E$ is a set of five proportional counters (called "Proportional Counter Array" or PCA) with large collecting area and very good time resolution $(\approx 1$ microsecond). Therefore, PCA is an ideal instrument to study the fast timing phenomena, such as burst oscillations (§ 3.1.6), accretion-powered pulsations ( $(3.2)$, kilohertz quasi-periodic oscillations ( $\S 3.3$ ), and also the continuum energy spectrum (e.g., $\S 3.1 .3)$. PCA is also the best instrument to study most of the properties of thermonuclear bursts (see $\S 3.1$ ). However, it is not suitable to study the spectral lines because of its poor energy resolution. This instrument operates in $2-60 \mathrm{KeV}$ band, although the effective collecting area becomes small above $\approx 20 \mathrm{keV}$. XMM-Newton satellite contains X-ray telescopes, chargecoupled devices and high resolution "Reflection Grating Spectrometers (RGS)". Therefore, this satellite, which operates in $\approx 0.2-12 \mathrm{keV}$ range, is ideal for spectral analysis, including narrow spectral lines. The Chandra observatory contains an X-ray telescope, charge-coupled devices, high resolution camera and transmission gratings. Its angular resolution ( $\sim$ subarcsecond) is the best among all the past and current X-ray instruments, and it is also ideal to detect and study narrow spectral lines (in $\approx 0.1-10$ $\mathrm{keV}$ range). XMM-Newton and Chandra are also useful to study the quiescent emission of neutron star LMXBs (§ 3.5). The Suzaku satellite also has X-ray telescopes and charge-coupled devices. Since it contains an additional "Hard X-ray Detector" (HXD), it can study the broadband energy spectrum. Therefore, Suzaku is very useful to observe the broad relativistic iron emission lines and the associated "disk reflection" spectra (see $\S 3.4$ ).

Here is the plan of this review. In $\S 2$, we describe the aim and the timeliness of this review. In $\S 3$, we briefly discuss a few observed properties of neutron star LMXBs, how these properties can be used to measure neutron star parameters, the relevant systematics and the plausible ways to reduce these systematic uncertainties. $\S 4$ mentions why LMXBs are useful to measure the neu- tron star parameters, and $\S 5$ includes a summary of this review, as well as a brief discussion on future prospects.

\section{Why This Review and Why Now?}

This review aims to describe the major neutron-starparameter-measurement methods for LMXB systems briefly, and to be useful as a quick reference for astrophysicists, nuclear physicists, particle physicists, and the scientists working in fluid mechanics, general theory of relativity, and other relevant fields. This is probably the first time that all the methods, including a recently established method based on broad relativistic spectral iron emission lines (see $\S 3.4 .2$ ), for LMXB systems have exclusively been compiled together. Although, this review does not include the techniques for all types of neutron star systems, the discussed methods form a complete set for the purpose of constraining EoS models. This is because at least three independent parameters of the same neutron star must be measured to constrain these models (see $\S 1$ ).

We believe that this review is timely, because of several new discoveries and advancement in the field of neutron star LMXBs made in the past few years. To mention a few, (1) Chakrabarty et al. (2003) established that the burst oscillations do give the neutron star spin frequency (see $\S 3.1 .6$ ); (2) the sample size of accretionpowered millisecond-period pulsars has increased from 1 to 12 (§ 3.2.1); (3) Cottam et al. (2002) reported the only plausible observation of the neutron star surface atomic spectral lines (see $\S 3.1 .4$ ); (4) Bhattacharyya and Strohmayer (2007b) established the inner accretion disk (or relativistic) origin of the broad iron line from a neutron star LMXB for the first time ( $\operatorname{see} \oint 3.4 .1$ ); and (5) Steeghs and Casares (2002) detected the companion star of an LMXB based on Bowen blend emission lines, and constrained the neutron star mass using these lines for the first time (see $\S 3.6$ ).

\section{Methods}

X-ray emission of an LMXB from a region close to the neutron star surface contains information about the stellar parameters. This information can be extracted from the observed spectral and timing properties of these Xrays. Here we discuss the properties of the following five $\mathrm{X}$-ray phenomena, and the methods for neutron star parameter measurement using them: (1) thermonuclear Xray bursts; (2) accretion-powered millisecond-period pulsations; (3) kilohertz quasi-periodic oscillations; (4) broad relativistic iron lines; and (5) quiescent emissions. In addition, we discuss a mass measurement technique based on the orbital motions of the binary components.

\subsection{Thermonuclear X-ray Bursts}

\subsubsection{What is a Thermonuclear X-ray Burst?}

Eruptions in X-rays are observed from many neutron star LMXB systems every few hours to days (Strohmayer 
and Bildsten , 2006; Galloway et al. , 2008a). These are called type-I X-ray bursts. The observed X-ray intensity sharply increases typically by a factor of $\sim 10$ in $\approx 0.5-5$ seconds, and then decreases relatively slowly in $\approx 10-100$ seconds during such a burst (Galloway et al. (2008a); see also Figure 2). The typical energy emitted in a few seconds is $\sim 10^{39}$ ergs (Strohmayer and Bildsten , 2006). These bursts were first discovered in 1970's (Grindlay et al. , 1976; Belian et al. , 1976). Soon after this discovery it was realized that they originate from intermittent unstable nuclear burning of accreted matter on the neutron star surfaces (Joss , 1977; Lamb and Lamb , 1978; Strohmayer and Bildsten , 2006). The neutron star surface origin was supported by the observational fact that the burst emission area matched with the expected surface area of a neutron star (Swank et al. , 1977; Hoffman et al. , 1977a). Note that the emission area can be estimated from the fitting of the energy spectra of the bursts. The unstable burning is required because, while the nuclear energy released per nucleon by fusion is only a few $\mathrm{MeV}$, the gravitational energy released by a nucleon falling on the neutron star surface from a large distance is $G M m_{\text {nucleon }} / R$ ( $M$ and $R$ are neutron star mass and radius respectively), which is typically $\sim 200 \mathrm{MeV}$. Therefore, if the nuclear burning were stable, the nuclear energy would be entirely lost in the observed gravitational energy, and the burst could not be powered by the nuclear energy release. This nuclear burning instability is driven by a nuclear energy generation rate which is more temperature sensitive than the radiative cooling. Such a "thin shell" instability was first theoretically discovered by Schwarzschild and Härm (1965) for the asymptotic giant branch phase of stellar evolution, and was shown to occur in the thin shell of hydrogen and helium on neutron star surfaces (Hansen and van Horn , 1975). But what shows that the nuclear burning causes the bursts? A primary evidence of it comes from the following observational fact. For a data set from a given source, the ratio of the total energy content in all the bursts to that in the non-burst emission roughly tallies with the ratio of the expected nuclear energy release per nucleon to the expected gravitational energy release per nucleon. This strongly suggests that the accreted matter accumulates on the neutron star surface for a long time, and then produces a burst via a quick thermonuclear burning. Therefore these bursts are known as "thermonuclear bursts" (see the reviews Lewin et al. (1993, 1995) and Strohmayer and Bildsten (2006) for detailed descriptions of these bursts).

For many neutron star LMXBs, the accreted material consists of mostly hydrogen, some helium and a small amount of heavier elements, although for ultra-compact Xray binaries (UCXBs) the donor companion is a hydrogen deficient and heavier element rich dwarf star (in't Zand et al. (2008) and references therein). The accreted matter, which accumulates on the neutron star surface, undergoes hydrostatic compression as more and more material piles on, and the ignition column density and temperature are

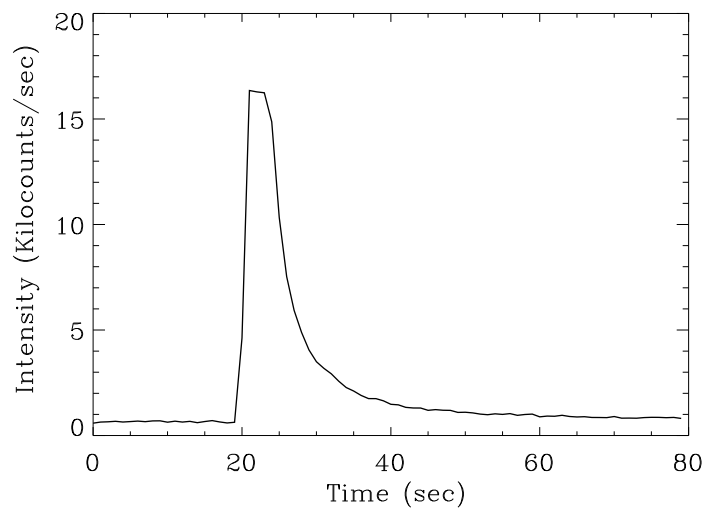

Figure 2: This figure shows the X-ray intensity profile of a typical thermonuclear X-ray burst observed from an LMXB. The burst light curve is for the entire RXTE PCA band, and with $1 \mathrm{sec}$ binsize. After the onset of the burst, the X-ray intensity increases sharply by more than an order of magnitude compared to the pre-burst intensity (powered by the gravitational potential energy), and then decays relatively slowly as the neutron star surface cools down (§ 3.1.1).

reached in a few hours to days (Schatz and Rehm, 2006). The burning typically happens at a depth of $\approx 10 \mathrm{~m}$ at a column density of $\sim 10^{8} \mathrm{gm} \mathrm{cm}^{-2}$. The compression rate and the ignition depends on the accretion rate, which sets four distinct theoretically expected regimes of burning (Bildsten , 2000).

Regime 1: The temperature of the accumulated material exceeds $10^{7} \mathrm{~K}$ in most cases, and hence the hydrogen burns via the CNO (carbon-nitrogen-oxygen) cycle instead of the pp (proton-proton) cycle. This causes mixed hydrogen and helium bursts triggered by the unstable hydrogen ignition, which happens for the accretion rate per unit neutron star surface area $\dot{\mathrm{m}}<900 \mathrm{gm} \mathrm{cm}^{-2} \mathrm{sec}^{-1}\left(Z_{\mathrm{CNO}} / 0.01\right)^{1 / 2}$ (Bildsten , 2000). Here $Z_{\mathrm{CNO}}$ is the mass fraction of CNO. Regime 2: the proton capture timescale becomes shorter than the subsequent $\beta$ decay lifetimes at higher temperatures $\left(T>8 \times 10^{7} \mathrm{~K}\right)$. Hence, the hydrogen burns via the "hot" CNO cycle of Fowler and Hoyle (1965):

$$
{ }^{12} \mathrm{C}(p, \gamma){ }^{13} \mathrm{~N}(p, \gamma){ }^{14} \mathrm{O}\left(\beta^{+}\right){ }^{14} \mathrm{~N}(p, \gamma){ }^{15} \mathrm{O}\left(\beta^{+}\right){ }^{15} \mathrm{~N}(p, \alpha){ }^{12} \mathrm{C}
$$

In this case, the hydrogen burning happens in a thermally stable manner (i.e., without triggering a thermonuclear burst) simultaneously with the accumulation of matter. This allows a helium layer to build up below the hydrogen layer. For the $\dot{\mathrm{m}}$ range $900 \mathrm{gm} \mathrm{cm}^{-2} \mathrm{sec}^{-1}$ $\left(Z_{\mathrm{CNO}} / 0.01\right)^{1 / 2}<\dot{\mathrm{m}}<2 \times 10^{3} \mathrm{gm} \mathrm{cm}^{-2} \mathrm{sec}^{-1}\left(Z_{\mathrm{CNO}} / 0.01\right)^{13 / 18}$, the hydrogen entirely burns before this helium is ignited (Bildsten , 2000; Strohmayer and Bildsten , 2006). So when the helium ignition happens, a short $(\sim 10 \mathrm{sec})$ but very intense burst occurs by the unstable triple-alpha reaction of the pure helium $\left(3 \alpha \rightarrow{ }^{12} \mathrm{C}\right)$. Such a burst is called a "helium burst". Many of these bursts are so intense that the local X-ray luminosity in the neutron star atmosphere may exceed the Eddington limit (for which the 
radiative pressure force balances the gravitational force), and the photospheric layers may be lifted off the neutron star surface. Such bursts are called photospheric radius expansion bursts (Strohmayer and Bildsten (2006) and references therein).

Regime 3: hydrogen burns via the "hot" CNO cycle in a stable manner for $\dot{\mathrm{m}}>2 \times 10^{3} \mathrm{gm} \mathrm{cm}^{-2} \mathrm{sec}^{-1}\left(Z_{\mathrm{CNO}} / 0.01\right)^{13 / 18}$ (as for the regime 2). But in this case, enough amount of unburnt hydrogen remains present at the time of helium ignition. This is because at this higher accretion rate, the helium ignition conditions are satisfied much sooner. Therefore in this regime, mixed hydrogen and helium bursts are triggered by the helium ignition. During such a burst, the thermal instability can produce elements beyond the iron group (Hanawa et al. , 1983; Hanawa and Fujimoto , 1984; Koike et al. , 1999; Schatz et al. , 2001) via the rp (rapid-proton) process of Wallace and Woosley (1981). This is because the triggering helium flash makes the temperature high enough to allow the "breakout reaction" ${ }^{15} \mathrm{O}(\alpha, \gamma){ }^{19} \mathrm{Ne}$ (Wallace and Woosley , 1981; Schatz et al. , 1999; Fisker et al. , 2006) from hot CNO cycle to proceed faster than the $\beta$ decays. ${ }^{19} \mathrm{Ne}$ can return to the hot $\mathrm{CNO}$ cycle via the chain of reactions (Cooper et al. , 2006):

${ }^{19} \mathrm{Ne}\left(\beta^{+} \nu\right){ }^{19} \mathrm{~F}(p, \alpha){ }^{16} \mathrm{O}(p, \gamma){ }^{17} \mathrm{~F}(p, \gamma){ }^{18} \mathrm{Ne}\left(\beta^{+} \nu\right){ }^{18} \mathrm{~F}(p, \alpha){ }^{15} \mathrm{O}$. But if ${ }^{19} \mathrm{Ne}$ captures a proton by the reaction ${ }^{19} \mathrm{Ne}(p, \gamma){ }^{20} \mathrm{Na}$, the nuclear reactions go out of the hot CNO cycle loop, and follow the rp process. This process burns hydrogen via successive proton capture and $\beta$ decays, and produces a large range of heavy nuclei (Schatz et al. , 1999, 2001). The long series of $\beta$ decays makes these bursts typically much longer $(\sim 100 \mathrm{sec})$ than the helium bursts.

Regime 4: finally at a very high accretion rate (comparable to the Eddington limit), the helium burning temperature sensitivity becomes weaker than the cooling rate's sensitivity (Ayasli and Joss , 1982; Taam et al. , 1996). Therefore in this regime, the stable burning sets in, and the thermonuclear bursts do not occur. However, recently Cooper and Narayan (2006) have shown that the bursts should not occur even if the accretion rate is more than $30 \%$ of the Eddington accretion rate, in case ${ }^{15} \mathrm{O}(\alpha, \gamma){ }^{19} \mathrm{Ne}$ reaction rate is lower than usually assumed. This appears to agree with observations (Cooper and Narayan, 2006). Finally, Cooper and Narayan (2007a) have suggested that for rapidly spinning neutron stars, burning instability sets in (i.e., bursts ignite) preferentially near the equator at low values of accretion rate and off of the equator at higher accretion rates.

Apart from usual type-I X-ray bursts, bursts of longer durations have been observed from several sources (e.g., Kaptein et al. (2000); in't Zand et al. (2002, 2005)). They have typical durations of $\approx 30$ minutes and energies $\approx 10^{41}$ ergs. These bursts are known as long bursts. Even longer and more energetic thermonuclear bursts, viz. superbursts, have been observed from a few neutron star LMXBs (Cornelisse et al. (2000); Kuulkers (2004); in't Zand et al. (2004); Kuulkers (2005); Keek et al. (2008) and references therein). The typical released energy, recurrence time and decay time of these bursts are $\sim 10^{42} \mathrm{ergs}$, $\sim$ years and $\sim 1-3$ hours respectively (Cumming and Bildsten , 2001; Strohmayer and Brown , 2002; Cooper et al. , 2009). Thermally unstable ${ }^{12} \mathrm{C}$ fusion (Woosley and Taam , 1976; Taam and Picklum , 1978; Brown and ${ }^{18}$ Bildsten , 1998) at a column depth of $\sim 10^{12} \mathrm{gm} \mathrm{cm}^{-2}$ may cause the superbursts (Cumming and Bildsten, 2001; Strohmayer and Brown , 2002). But this explanation has a problem for $\mathrm{H} / \mathrm{He}$ accretors, because only a small amount of carbon is expected to remain after the burning of $\mathrm{H}$ and He via the rp-process. Although several solutions of this problem have been proposed by Cumming and Bildsten (2001); Cooper et al. (2006); Cooper et al. (2009), more detailed theoretical studies are required to understand the origin of superbursts.

The study of thermonuclear bursts provides a unique opportunity to understand some aspects of extreme physics. In particular, these bursts can be very useful to constrain the neutron star parameters (see later sections), and hence to understand the nature of the stellar core matter at supranuclear densities (see $\S 1$ ). However, in order to use these bursts as a reliable tool, they have to be understood well (see, for example, Woosley et al. (2004); Weinberg et al. (2006a,b); Lin et al. (2006); Heger et al. (2007a); Cooper and Narayan (2007b); Fisker et al. (2008) for recent burst model calculations). One of the poorly explored aspects of these bursts is the thermonuclear flame spreading on the neutron star surfaces. It is expected that the accreted matter quickly spreads all over the neutron star surface (Inogamov and Sunyaev, 1999). Therefore a burst may ignite at a certain location on the stellar surface (Cooper and Narayan, 2007a; Maurer and Watts , 2008), and then the burning region may expand to cover the entire surface (Fryxell and Woosley , 1982; Spitkovsky et al. , 2002). The study of this flame spreading is not only essential to understand the bursts, but also important to use these bursts as a tool to probe the neutron star parameters (§ 3.1.6). However, the theoretical modeling of thermonuclear flame spreading including all the main physical effects is extremely difficult, and only recently Spitkovsky et al. (2002) have provided some insights in this field. Although these authors have ignored several physical effects (magnetic field, strong gravity, etc.) in their model, they have considered the effects of the Coriolis force, which is important as the bursting neutron stars are rapidly spinning (typical spin frequency $\sim 300-600 \mathrm{~Hz}$ ). The observational study of flame spreading is also no less difficult than the theoretical study. This is because, this spreading mostly happens within $\approx 1$ sec during the burst rise, and in order to study it observationally, one needs to detect and measure the evolution of the burst spectral and timing properties during this short time. Therefore, although the observational indications of flame spreading were found about a decade ago (e.g., Strohmayer et al. (1997b)), only recently such spreading could be confirmed and measured for a few bursts by analyzing the RXTE PCA data (Bhattacharyya 
and Strohmayer , 2005, 2006a,b,c, 2007a,c). These findings provide the motivation for rigorous theoretical studies including all the main physical effects, as well as for detailed analysis of the burst rise data.

\subsubsection{Why are Thermonuclear X-ray Bursts Useful?}

Thermonuclear X-ray Bursts can be very useful to constrain the neutron star parameters due to the following reasons.

(1) They originate from the neutron star surfaces. Therefore, the stellar parameters (e.g., mass, radius, spin frequency) influence the burst spectral and timing properties. Hence the modeling of these properties can be used to measure several neutron star parameters simultaneously (see later).

(2) Various burst phenomena, such as photospheric radius expansion, thermonuclear flame spreading, etc., can be useful to constrain the neutron star parameters (see later).

(3) Relatively low magnetic fields $\left(\sim 10^{7}-10^{9} \mathrm{G}\right)$ of the bursting neutron stars simplify the modeling of the surface emission.

(4) The surface emission during a burst is typically about 10 times brighter than the rest of the X-ray emission. Hence the surface emission can be isolated from the total emission relatively easily, and can be used to constrain the neutron star parameters with confidence. Note that this is not true for the non-burst emission, for which it is difficult to distinguish between the surface and the nonsurface emissions with sufficient accuracy.

(5) The $\sim 10$ times higher intensity of bursts, relative to the non-burst intensity, gives a significantly higher signalto-noise ratio.

(6) Many bursts can be observed from the same neutron star. The values of neutron star mass, radius and spin frequency, which affect the burst properties, do not change from one burst to another for a given source. Therefore, the joint analysis of many bursts, keeping the model values of these stellar parameters same for all the bursts, can be very useful to constrain these parameters. This technique is demonstrated in Bhattacharyya et al. (2005) (see also $\S 3.1 .8)$.

(7) Thousands of thermonuclear X-ray Bursts have been observed from about 80 sources (Liu et al. (2007); see also Galloway et al. (2008a) for a catalog of RXTE PCA bursts). Therefore, a large amount of burst data is already available.

\subsubsection{Continuum Spectrum Method}

The continuum spectra of thermonuclear X-ray bursts are typically quite well described with a blackbody model (Swank et al. (1977); Hoffman et al. (1977b); Galloway et al. (2008a); see also Figure 3). The temperature of this blackbody increases rapidly as the accumulated matter burns during the burst intensity rise, and then decreases relatively slowly as the neutron star surface cools down during the burst decay. If the entire neutron star surface emits like a blackbody of the same temperature at a given time, then the inferred radius at infinity $\left(R_{\infty}\right)$ of the neutron star can be obtained from the relation:

$$
R_{\infty}=\left(F_{\infty} / \sigma T_{\infty}^{4}\right)^{1 / 2} d
$$

Here $F_{\infty}$ is the observed bolometric flux, $T_{\infty}$ is the fitted blackbody temperature, $d$ is the source distance and $\sigma$ is the Stefan-Boltzmann constant. This provides one of the very few ways to measure the neutron star radius. Note that the radius measurement is typically more difficult than the measurements of the neutron star mass and spin frequency. This is because, whereas the gravitational effect of mass influences the observed motion of the companion star in a binary system, and the observed flux may be modulated by the neutron star spin, the radius exhibits no such effects. As a result, although the mass and the spin frequency of several neutron stars have been measured (Thorsett and Chakrabarty , 1999; Lamb and Boutloukos, 2008), accurate measurements of the radius are challenging.

The burst continuum spectrum method was used to constrain the neutron star radius soon after the discovery of these bursts (e.g., van Paradijs (1978); Goldman (1979); van Paradijs (1979, 1982); Marshall (1982); van Paradijs and Lewin (1986); Fujimoto and Taam (1986); Sztajno et al. (1987); Gottwald et al. (1987)). However, these efforts were not too successful because of the following systematic uncertainties.

(1) Equation 2 assumes that the entire stellar surface emits during the burst. If that is not true, this equation will underestimate the value of the neutron star radius.

(2) The lack of knowledge of the source distance introduces an uncertainty in the neutron star radius value inferred from this method.

(3) The surface gravitational redshift $(1+z)$ makes the observed radius $R_{\infty}$ and the actual neutron star radius $R_{\mathrm{BB}}$ different by an unknown factor.

(4) Although a blackbody model fits the observed burst spectrum well, this spectrum cannot be the blackbody originated from the burning layer. This is because the scattering of photons by the electrons and the frequency dependence of the opacity in the neutron star atmosphere hardens the energy spectrum (London et al. , 1984, 1986; Syunyaev and Titarchuk , 1986; Ebisuzaki and Nakamura, 1988; Titarchuk , 1994; Madej et al. , 2004; Majczyna et al. , 2005). Such a hardening shifts the burning layer blackbody spectrum towards the higher energies, and changes its shape slightly. This modified spectrum is called a "diluted blackbody", and its temperature $\left(T_{\text {col }}\right.$, i.e., the color temperature) is related to the actual blackbody temperature of the burning layer $\left(T_{\mathrm{BB}}\right)$ by an unknown color factor $f$. Consequently, the observed radius is lower than the actual radius by the square of the color factor. As a result, the actual temperature $\left(T_{\mathrm{BB}}\right)$ and radius $\left(R_{\mathrm{BB}}\right)$ are related to the observed values by the following relations (including the effects of the gravitational redshift; Sztajno et al. 


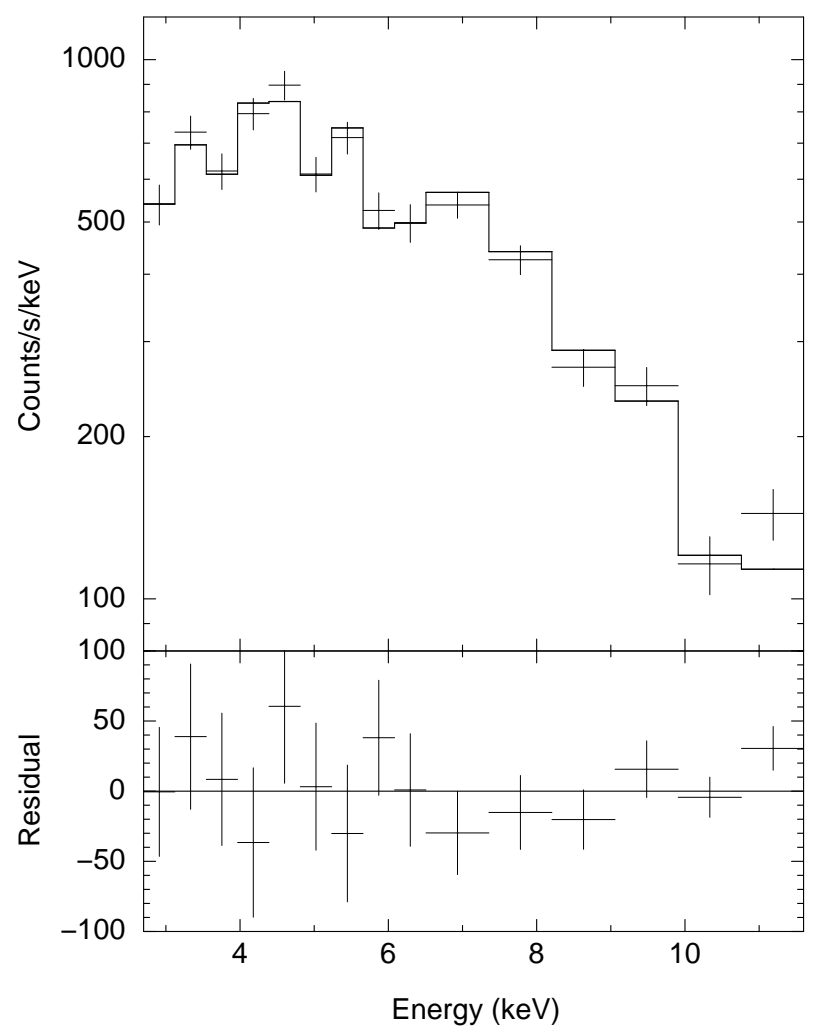

Figure 3: This figure shows the fitting of a thermonuclear burst spectrum with an absorbed blackbody model. The spectrum is for a $1 \mathrm{sec}$ segment of a burst observed from a neutron star LMXB 4U 1636-536 with $R X T E$ PCA. Since PCA cannot reliably measure the absorption due to the Galactic neutral hydrogen because such absorption mostly happens outside the PCA band, we fixed the neutral hydrogen column density $n_{\mathrm{H}}$ to the estimated value $\left(3.58 \times 10^{21} \mathrm{~cm}^{-2}\right)$ in the source direction for the fitting. The upper panel shows the data points with error bars, and the best-fit model, while the lower panel shows the (data-model). This figure demonstrates that the continuum spectra of thermonuclear X-ray bursts are typically well described with a blackbody model (§ 3.1.3).

$(1985))$ :

$$
\begin{aligned}
& T_{\mathrm{BB}}=T_{\infty} \cdot(1+z) / f ; \\
& R_{\mathrm{BB}}=R_{\infty} \cdot f^{2} /(1+z) ;
\end{aligned}
$$

where the surface gravitational redshift $1+z=\left(1-2 G M / R c^{2}\right)$ for a non-spinning neutron star. Here, $R / M$ is the neutron star radius-to-mass ratio, $G$ is the gravitational constant and $c$ is the speed of light in vacuum.

As mentioned earlier, thermonuclear bursts provide one of the very few promising methods to measure the neutron star radius. Therefore, although this method has a few systematic uncertainties, it is worthwhile to try to improve it by removing or at least reducing these uncertainties. Here we briefly mention a few plausible ways to reduce the uncertainties.

(1) Even when a part of the stellar surface burns, $R_{\infty}^{2}$ should be proportional to, and hence is a measure of the burning region area. Hence, the uncertainty \#1 should be

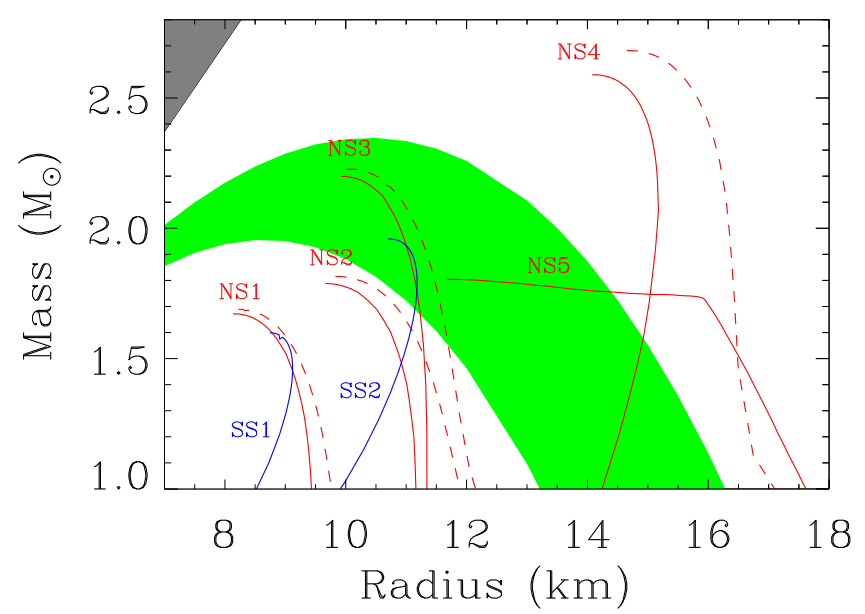

Figure 4: This figure shows the $M-R$ space of neutron stars with the curves corresponding to a few representative EoS models (same as Figure 1). The green patch shows the allowed $M-R$ space assuming $R_{\mathrm{BB}}=R$ and $1+z=\left(1-2 G M / R c^{2}\right)^{-1 / 2}$ in equation 4 , and for $R_{\infty} \cdot f^{2}$ in the range $15 \mathrm{~km}-18 \mathrm{~km}$ (see $\S 3.1 .3$ for details). This range should be reasonable, because $R_{\infty}$ is often found in the 10 $\mathrm{km}-20 \mathrm{~km}$ range, and $f$ can be within 1.2 and 1.8 according to Majczyna et al. (2005). This example figure demonstrates how the thermonuclear burst continuum spectra can be used to constrain the neutron star mass and radius, and hence the EoS models.

reduced if the burning region area can be estimated independently; for example, by fitting the phase-folded burst oscillation light curves with a physical model (see $\S 3.1 .6$ ). (2) In order to reduce the uncertainty \#2, one needs to measure the source distance by an independent method. This is possible if the source is in a globular cluster (e.g., Özel et al. (2009)); because the distances of these clusters can typically be measured with sufficient accuracy. The source distance can also roughly be measured from the flux of a photospheric radius expansion burst (see $\S 3.1 .5$ ). (3) The surface gravitational redshift $1+z$ depends on the stellar parameters. As mentioned earlier, for a nonspinning neutron star, $1+z=\left(1-2 G M / R c^{2}\right)^{-1 / 2}$. Therefore, the uncertainty $\# 3$ can be reduced if the neutron Stąr radius-to-mass ratio is constrained. Several observational features of neutron star LMXBs provide independent methods to measure this ratio (for example, see $\S 3.1 .4 ; \S 3.1 .6 ; \S 3.4 .2)$. However, if the uncertainties $\# 1$, \#2 and \#4 can be sufficiently reduced, it is possible to constrain the neutron star $M-R$ space effectively, even without measuring the $1+z$. This is shown in Figure 4, and can be understood from equation 4 using $1+z=\left(1-2 G M / R c^{2}\right)^{-1 / 2}$.

(4) The color factor $f$ depends on the neutron star surface gravity $g$, the actual blackbody temperature $T_{\mathrm{BB}}$ and the chemical composition in the stellar atmosphere (Madej et al. , 2004; Majczyna et al. , 2005). Therefore, in order to measure $f$ and to reduce the uncertainty \#4, one needs 
to estimate these three parameter values, and model the atmosphere for these values. While the gravity $g$ depends on the neutron star mass and radius (see equation 7 ; see $\S 3.1 .7$ and Figure 10 for a plausible method to measure $g$ ), the latter two parameter values can be estimated from a realistic modeling of the thermonuclear bursts and the chemical composition of the burning layer. The determination of the composition may be attempted using the following ways. (a) The observed duration of the burst, as well as the detailed modeling of its light curve and other properties may be useful to determine the chemical composition (see $\S 3.1 .1$; also see Woosley et al. (2004); Heger et al. (2007a). (b) The observed nature of the companion star can be used to constrain the chemical composition of the accreted matter. Note that both the preburst burning layer and the preburst atmosphere consist of this matter, although the former may additionally contain ashes from the previous bursts. In case the companion is not directly observable, the measured binary orbital period may be useful to probe its nature. For example, if the system is an UCXB (orbital period < 80 min; in't Zand et al. (2008)), the companion and hence the accreted matter should be hydrogen deficient and heavier element rich (see $\S 3.1 .1$ ). (c) The observed atomic spectral lines from the non-burst emission can also be useful to estimate the composition of the accreted matter. Such lines are normally observed from high-inclination neutron star LMXBs (e.g., JimenezGarate et al. (2003); Díaz Trigo et al. (2006)). Therefore, the detailed observational and theoretical studies of burst and non-burst emissions can, in principle, constrain the burst color factor $f$. However, note that $f$ is expected to change, as $T_{\mathrm{BB}}$ and plausibly the atmospheric chemical composition evolve during a burst, and hence one needs to estimate $f$ vaules throughout the burst.

The burning region is normally expected to cover the entire stellar surface during the burst decay (see $\S 3.1 .1$ ). If this is true, the uncertainty \#1 does not appear, and the continuum spectrum of burst decay (rather than burst rise) should be more useful to measure the neutron star radius. But unfortunately, the inferred burning area $\left(\propto R_{\infty}^{2}\right)$ can increase, decrease, or go up and down erratically in a given burst (see Figure 9 of Galloway et al. (2008a)). This may be because of the actual change of $R_{\mathrm{BB}}^{2}$ and/or the evolution of $f$ (see equation 4 ) and/or any other reasons. Therefore, it is essential to understand the apparently erratic evolution of $R_{\infty}^{2}$ for a reliable measurement of neutron star radii using burst decay continuum spectra. Very recently, Bhattacharyya et al. (2009) have, for the first time, reported a pattern in the apparently erratic behaviour of the inferred burning area. They found that the rate of change of the inferred area during decay is anticorrelated with the burst decay duration, and this anticorrelation is significant at the $<10^{-45}$ level (see Figure 5). These authors have suggested that the variations in $f$, and more specifically the variations in the composition of the atmosphere between bursts with long and short durations, may explain the anticorrelation. Such a varia-

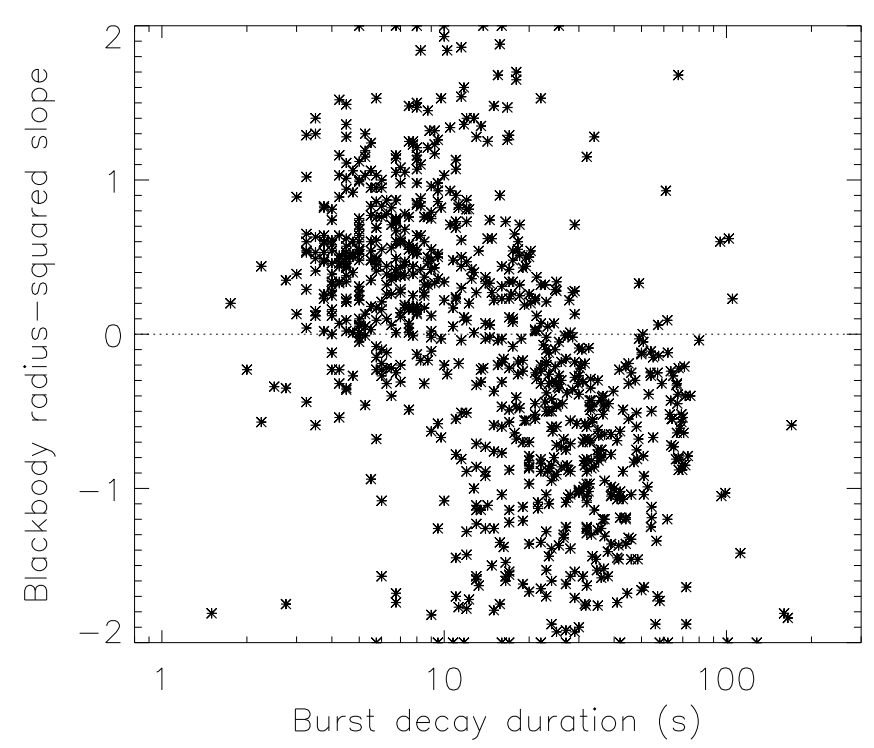

Figure 5: Slope of burst blackbody radius-squared $\left(R_{\infty}^{2} \propto\right.$ inferred burning area) during burst decay vs. burst decay duration. Each point corresponds to one burst, and 877 bursts from 43 sources have been shown. This figure indicates a strong anticorrelation (significant at the $<10^{-45}$ level) between the rate of change of the inferred burning area and the decay duration, which can be useful to make the continuum burst spectrum method reliable (see § 3.1.3). For the exact definition of the radius-squared slope, see Bhattacharyya et al. (2009).

tion can be reasonable, because the duration of a burst depends on the chemical composition of the burning material (see $\S 3.1 .1$ ). However, in order to verify this model, extensive theoretical study is required. But, whatever is the correct model, the newly discovered pattern in the $R_{\infty}^{2}$ evolution will play an extremely important role to make the continuum spectrum method reliable.

\subsubsection{Spectral Line Method}

Observation and identification of narrow atomic spectral lines from the surface of a neutron star (for example, during a thermonuclear burst) provide the cleanest way to measure the stellar radius-to-mass ratio using the following formula (for the Schwartzschild spacetime appropriate for a non-spinning neutron star): $E_{\mathrm{em}} / E_{\mathrm{obs}}=$ $1+z=\left[1-\left(2 G M / R c^{2}\right)\right]^{-1 / 2}$ (Özel and Psaltis (2003); Bhattacharyya et al. (2006a); see Figure 6). Here, $E_{\mathrm{em}}$ is the emitted energy of the line photons, and $E_{\mathrm{obs}}$ is the observed energy of these photons. However, if the surface lines are broad and asymmetric, $E_{\text {obs }}$ cannot be determined uniquely, and hence it is difficult to measure $R c^{2} / G M$ accurately using the above formula. Unfortunately, neutron stars in LMXB systems typically spin with high frequencies $(\sim 300-600 \mathrm{~Hz})$, and hence the Doppler effect and the special relativistic beaming are expected to make the surface lines broad and asymmetric. Recently, Bhattacharyya et al. (2006a) have suggested a way to 
measure the stellar $R c^{2} / G M$ using the above formula for such broad lines. They have shown that using $\sqrt{E_{\mathrm{l}} E_{\mathrm{h}}}$ as $E_{\text {obs }}$, one can measure $R c^{2} / G M$ with less than $2 \%$ error. Here, $E_{\mathrm{l}}$ is the energy of the low energy end of the broad line, and $E_{\mathrm{h}}$ is that of the high energy end. This shows that surface lines can be used to accurately measure the stellar $R c^{2} / G M$ even for LMXBs having rapidly spinning neutron stars.

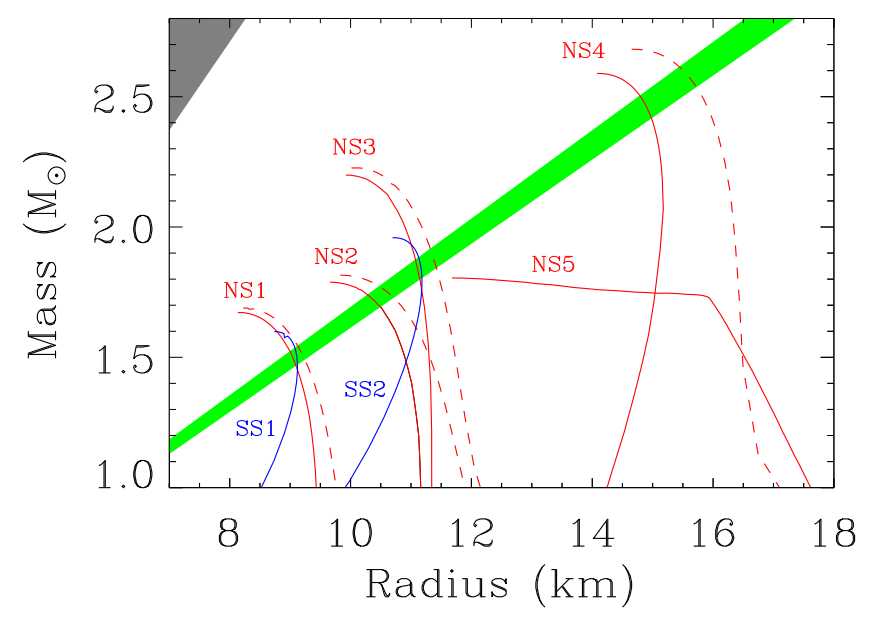

Figure 6: This figure shows the $M-R$ space of neutron stars with the curves corresponding to a few representative EoS models (same as Figure 1). The green patch shows the allowed $M-R$ space for a reasonable range of the radius-to-mass ratio $\left(R c^{2} / G M=4.0-4.2\right)$. This example figure demonstrates how the measured radius-to-mass ratio can be used to constrain the neutron star $M-R$ space (see $\S 3.1 .4 ; \S 3.1 .6$; and $\S 3.4 .2$ for details).

A surface absorption line originated from the entire stellar surface can have only one dip (or peak for an emission line; see Figure 1 of Özel and Psaltis (2003)). On the other hand, if it originates from a portion of the surface, especially near the equator, it can have two dips (or two peaks for an emission line; Özel and Psaltis (2003); Bhattacharyya et al. (2006a); see also Figure 7). In either case, its width and shape depends on the neutron star parameter values (Figure 7; see also Özel and Psaltis (2003); Bhattacharyya et al. (2006a)). Therefore, fitting the surface lines with physical models can be useful to constrain additional (apart from $R c^{2} / G M$ ) neutron star parameters.

Surface lines are likely to be detected and identified from thermonuclear X-ray bursts and hence from neutron star LMXB systems. This is because: (1) the continuous accretion and the radiative pressure may keep the lineforming heavy elements in the stellar atmosphere for the time-duration required for the line detection; (2) nuclear burning ashes may be transported to the atmosphere by strong convection (Weinberg et al. , 2006a); (3) the relatively low magnetic fields ( $\sim 10^{7}-10^{9}$ Gauss) of the neutron stars in LMXBs should make the line identification easier by keeping the magnetic splitting negligible; and (4) the luminous bursts give good signal-to-noise ratio. Moreover, the modeling of the surface lines detected from thermonuclear bursts may be easier, because (1) magnetic field is relatively low; and (2) during the bursts, typically $90 \%$ of the total emission originates from the neutron star surface, and hence the surface spectral line should be largely free from the uncertainty due to the other X-ray emission components, such as the accretion disk.

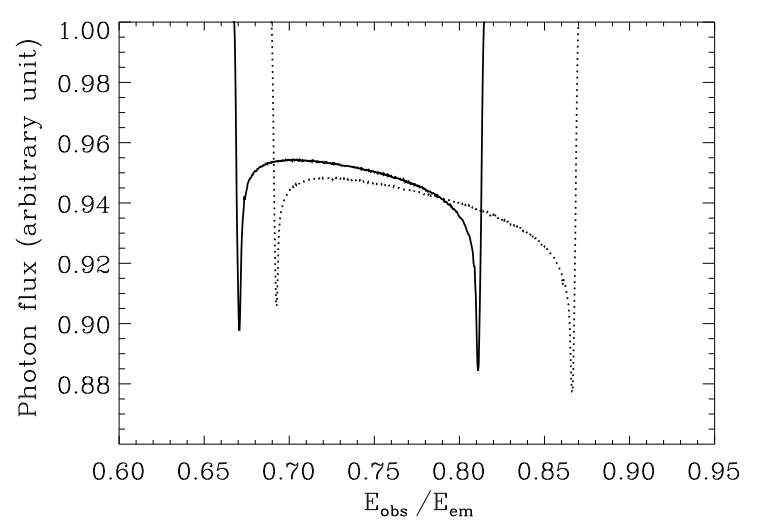

Figure 7: Profiles of narrow absorption lines formed at the equatorial portion of the neutron star surface as they would appear to an observer at $i_{\text {spin }}=75^{\circ}$, where $i_{\text {spin }}$ is the observer's inclination angle measured from the stellar spin axis (see Bhattacharyya et al. $(2006 \mathrm{a}))$. Here, $E_{\text {em }}$ is the emitted energy, $E_{\text {obs }}$ is the observed energy, and the continuum is assumed to be at a constant photon flux of 1 (in arbitrary unit). The solid profile is for the neutron star parameter values $M=1.4 M_{\odot}$ and $R=9 \mathrm{~km}$; and the dotted profile is for $M=1.5 M_{\odot}$ and $R=11 \mathrm{~km}$. All other source parameter values are same, including the stellar spin frequency $(=400 \mathrm{~Hz})$. This figure shows that the shape and the width of the surface spectral lines depend on the neutron star parameters ( $\S 3.1 .4$ ).

In fact, the only plausible observation of the neutron star surface atomic spectral lines was from an LMXB EXO 0748-676 (Cottam et al. , 2002). These authors detected two significant absorption features in the XMM-Newton Reflection Grating Spectrometers (RGS) energy spectra of the thermonuclear X-ray bursts, which they identified as Fe XXVI and Fe XXV $n=2-3$ lines with a gravitational redshift of $1+z=1.35$. In order to detect these lines, they had to combine the spectra of 28 bursts. The absorption of some of the burst photons (originated in deep burning layers) by the iron ions in the upper atmosphere might give rise to these redshifted lines. Chang et al. (2005) showed that the observed strength of the iron lines could be produced by a neutron star photospheric metallicity, which was $2-3$ times larger than the solar metallicity (see also Chang et al. (2006)). The identification of these two lines as the surface atomic spectral lines is reasonable for the following reasons: (1) the early burst phases (when the temperature was higher) showed the Fe XXVI line, while the late burst phases (when the temperature was lower) exhibited the Fe XXV line, which is consistent 
with the qualitative temperature dependence of the iron ionization states; (2) the narrow lines were consistent with the relatively slow stellar spin rate $(45 \mathrm{~Hz})$, reported by Villarreal and Strohmayer (2004) (but see later); and (3) both the lines showed the same surface gravitational redshift, and this redshift value is reasonable for the surface of a typical neutron star. However, recent observations have argued against the neutron star surface origin of these two lines. (1) these lines were not significantly detected from the later observations of EXO 0748-676 (Cottam et al. , 2008). However, such disappearence might be caused by the change in the photospheric conditions, which might weaken the lines. (2) Galloway et al. (2009) have discovered burst oscillations at $552 \mathrm{~Hz}$ from EXO 0748-676 with a significance of $6.2 \sigma$. This argues that the neutron star spin frequency is $552 \mathrm{~Hz}$ (see $\S 3.1 .6$ ). If this is true, the observed narrow spectral lines from EXO 0748-676 could originate from the stellar surface only if the entire line forming region were very close to the neutron star spin axis (Bhattacharyya et al. , 2006a). Moreover, recently Balman (2009) has suggested that the $45 \mathrm{~Hz}$ frequency reported by Villarreal and Strohmayer (2004) may be a variability within the boundary layer. Therefore, the surface origin of the observed absorption features from EXO 0748676 is not established yet. Besides, Kong et al. (2007) did not find surface spectral lines from the combined $X M M$ Newton RGS spectra of 16 thermonuclear bursts from the neutron star LMXB GS 1826-24.

\subsubsection{Photospheric Radius Expansion Burst Method}

As mentioned in $\S 3.1 .1$, for some of the strong bursts, viz. photospheric radius expansion (PRE) bursts, the photospheric radiative pressure force exceeds the gravitational force, and the photosphere is temporarily lifted off the neutron star surface. During the expansion of the photosphere, the temperature decreases and the burning area inferred from the continuum spectroscopy increases. Subsequently, as the photosphere comes down, the temperature increases and the inferred burning area decreases. After the photosphere settles on the stellar surface at the touchdown time, it starts cooling down, and the temperature decreases again. As a result, PRE bursts can be identified by two maxima in the temperature profile and a burning area maximum corresponding to the temperature minimum (see Figure 9 of Galloway et al. (2008a)). The amount of photospheric expansion can vary by a large extent from burst to burst. Normally during the expansion, the X-ray flux does not change much. But for certain very powerful bursts the expansion can be so large that the photospheric temperature may be shifted below the X-ray band, and the burst may be divided into two parts: a precursor and a main part (Lewin et al. , 1984; Strohmayer and Brown , 2002). For other somewhat less powerful PRE bursts, a part of the flux may shift out of the X-ray band making the burst intensity profile doublepeaked (Strohmayer and Bildsten , 2006).

The Eddington limit (see $\S 3.1 .1$ ) on the neutron star

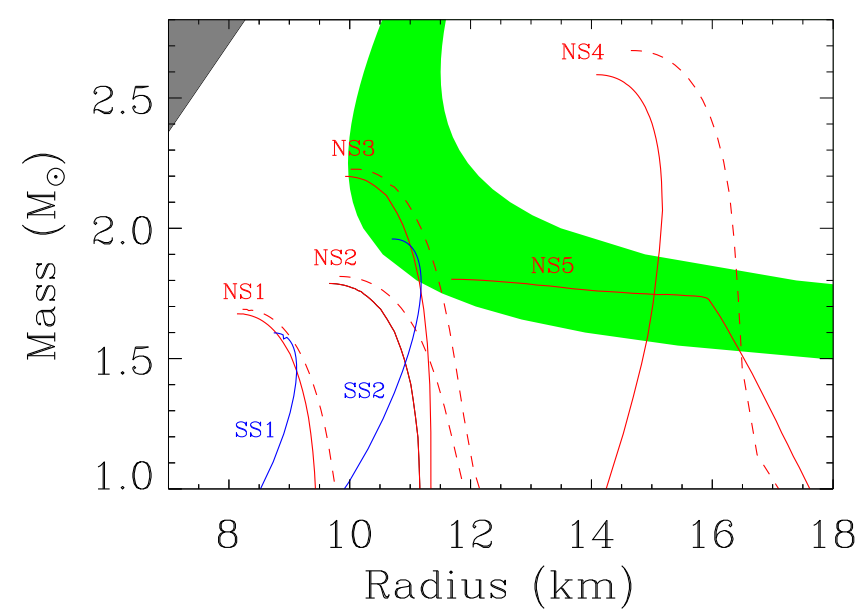

Figure 8: This figure shows the $M-R$ space of neutron stars with the curves corresponding to a few representative EoS models (same as Figure 1). The green patch shows the allowed $M-R$ space using equation 6 (see $\S 3.1 .5$ for details). In this example figure we have ensured that the green patch passes through the the reasonable ranges of $M$ and $R$, and this figure demonstrates how the photospheric radius expansion bursts can be used to constrain the neutron star mass and radius, and hence the EoS models.

surface is given by

$$
L_{\mathrm{Edd}}=(4 \pi G M c / \kappa)\left(1-2 G M / R c^{2}\right)^{-1 / 2}=4 \pi R^{2} \sigma T_{\mathrm{BB}}^{4},
$$

where, $\kappa$ is the atmospheric opacity (e.g., Strohmayer and Bildsten (2006)). Here we have assumed that the entire stellar surface emits, and the surface gravitational redshift $1+z=\left[1-\left(2 G M / R c^{2}\right)\right]^{-1 / 2}$, which is appropriate for a non-spinning neutron star. It may be reasonable to identify the observed touchdown flux as the Eddington-limited flux (Özel , 2006). Therefore, equations 5 and 3 for the touchdown time can be used to constrain the neutron star $M-R$ space, if the entire stellar surface emits (see Galloway et al. (2003)) and the parameters $\kappa$ and color factor $f$ are known. The flux observed at infinity corresponding to equation 5 is (e.g., Özel (2006))

$$
F_{\text {Edd }}=\left(1 / 4 \pi d^{2}\right)(4 \pi G M c / \kappa)\left(1-2 G M / R c^{2}\right)^{1 / 2}
$$

where $d$ is the source distance. Considering the observed touchdown flux as $F_{\text {Edd }}$ (Özel , 2006), equation 6 can be used to constrain the neutron star $M-R$ space (see Figure 8), if the entire stellar surface emits (Galloway et al. , 2003) and the parameters $d$ and $\kappa$ are known. In order to estimate $\kappa$ and/or $f$, one needs to model the stellar atmosphere for a known chemical composition. The possible ways to determine this composition is discussed in $\S 3.1 .3$. Apart from the above methods, the observed variation of the Eddington luminosity and the corresponding change in the gravitational redshift caused by the photospheric expansion may, in principle, be useful to determine the neutron star surface gravitational redshift (see for example, 
Damen et al. (1990); van Paradijs et al. (1990)). These and related methods have been used by many scientists in order to constrain the neutron star mass and radius (e.g., Fujimoto and Taam (1986); Ebisuzaki (1987); Sztajno et al. (1987); van Paradijs and Lewin (1987); Chevalier and Ilovaisky (1990); Kaminker et al. (1990); Damen et al. (1990); van Paradijs et al. (1990); Haberl and Titarchuk (1995); Smale (2001); Strohmayer et al. (1998); Titarchuk and Shaposhnikov (2002); Shaposhnikov et al. (2003); Shaposhnikov and Titarchuk (2004); Özel (2006)). However, so far such measurements heve not been generally precise enough, because of the systematic uncertainties primarily due to the unknown parameter values mentioned above (e.g., Strohmayer and Bildsten (2006)).

This and other sections (e.g., $\S 3.1 .3 ; \S 3.5 .2)$ point out that the knowledge of the source distance $d$ can be very useful to measure the neutron star parameters. In 1978, van Paradijs (1978) suggested that the photospheric expansion flux, which corresponds to the Eddington flux, can be a "standard candle", and hence a distance indicator (e.g., using equation 6). In the next 30 years, scientists have tested this idea by examining the variation of the photospheric expansion flux from burst to burst, comparing the inferred distance to the known distance (whenever available; e.g., for globular cluster sources), and in other ways (e.g., van Paradijs (1981); Basinska et al. (1984); Smale (1998); Kuulkers and van der Klis (2000); Kuulkers et al. (2003); Galloway et al. (2003, 2006); Galloway and Cumming (2006); Özel (2006); Galloway et al. (2008b); Table 9 of Galloway et al. (2008a)). They have found that this method is affected by the uncertainties due to unknown neutron star mass, surface gravitational redshift, photospheric composition, etc., and hence the distance can typically be constrained within a somewhat large range.

\subsubsection{Burst Oscillation Method}

High frequency narrow timing features, viz. burst oscillations, have been detected from many thermonuclear Xray bursts with the PCA instrument of $R X T E$ (Strohmayer and Bildsten , 2006). They can be observed either in the burst rising part or in the decay part, or in both. However, neither these oscillations appear in every burst from a given source, nor they have been observed from every neutron star LMXB (Galloway et al. , 2008a). Burst oscillations were first discovered from the LMXB 4U 1728-34 by Strohmayer et al. (1996) in 1996. After that, this feature has been detected from 20 other neutron star LMXBs (oscillations from three of them are not yet confirmed; see Bhattacharyya (2007); Lamb and Boutloukos (2008); Galloway et al. (2009); Watts et al. (2009a); Markwardt et al. (2007); Watts et al. (2009b) and references therein).

Burst oscillations can be seen as a peak in the Fourier power spectrum computed from a high time resolution light curve of either the entire burst or a part of it (see Figure 9). The frequency of this feature can evolve during a burst (Strohmayer and Bildsten (2006) and references therein). Normally frequency shifts by less than $1 \%$ of the
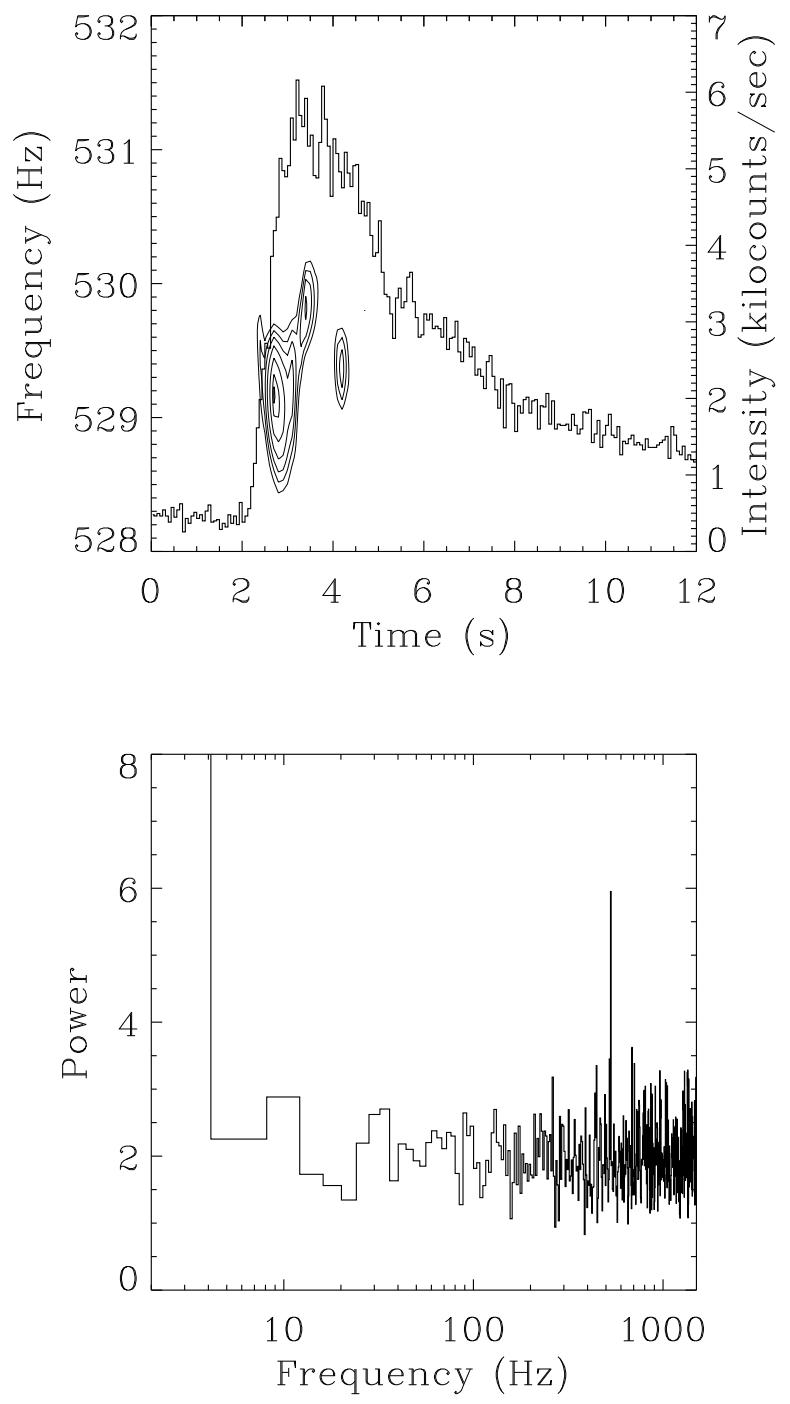

Figure 9: Upper panel: X-ray intensity profile of a thermonuclear Xray burst from the neutron star LMXB 1A 1744-361 (Bhattacharyya et al. , 2006b). Power contours around $530 \mathrm{~Hz}$ implies that there are significant intensity variations (burst oscillations; $§ 3.1 .6$ ) with $\approx 530$ $\mathrm{Hz}$ frequency. Note that the shape of the power contours indicates a slight change in frequency with time, which is common for burst oscillations. Lower panel: The corresponding power spectrum. The peak at $\approx 530 \mathrm{~Hz}$ implies the burst oscillations with that frequency. 
mean frequency, but it can be somewhat greater for some bursts. The frequency normally increases from a lower initial value to an asymptotic value, but it has also been seen to decrease for some bursts (see for example, Muno et al. (2002a)). It is believed that burst oscillations originate from an azimuthally asymmetric brightness pattern on the spinning neutron star surface. Since in such a case the Xray flux measured by the observer should vary with the stellar spin frequency, the burst oscillation (asymptotic) frequency is believed to be the stellar spin frequency. This model is established because of the following reasons.

(1) Burst oscillations originate from the thermonuclear bursts which are neutron star surface phenomena.

(2) The oscillation (asymptotic) frequency is very stable on timescales of years for a given source (Muno et al. , 2002a). This strongly suggests that burst oscillations are connected to the stellar spin, which is also expected to be a stable property.

(3) The observed burst oscillation frequencies are mostly in the range $\sim 300-600 \mathrm{~Hz}$. Such rapid spin rates are expected for neutron stars in LMXBs, because of the accretioninduced angular momentum transfer $(\S 1)$.

(4) Spin frequencies of five burst oscillation sources are independently known from accretion-powered pulsations (see $\S 3.2$ ). For each of them the burst oscillation frequency matches the known frequency well (Chakrabarty et al. , 2003; Strohmayer et al. , 2003; Casella et al. , 2008; Watts et al. , 2009a,b).

The evolution of burst oscillation frequency may be caused by the slow motion of the photosphere on the stellar surface. One reason for such a slow motion may be the angular momentum conservation of contracting thermonuclear shells (Strohmayer et al. , 1997a). In this scenario, a thermally expanded shell has a spin frequency lower than the stellar spin frequency due to angular momentum conservation. As the shell contracts, its spin frequency increases and tends to the stellar spin frequency. However, this model cannot explain a frequency shift roughly larger than $2 \mathrm{~Hz}$ (Strohmayer and Bildsten , 2006) observed from several bursts (e.g., Galloway et al. (2001); Wijnands et al. (2001); Chakrabarty et al. (2003)). Therefore, other physical reasons (e.g., involving magnetic field; Bhattacharyya and Strohmayer (2006c); Heng and Spitkovsky (2009)) may contribute to the frequency evolution process.

Burst oscillations provide one of the two ways to measure the spin rates of neutron stars in LMXB systems (see $\S 3.2$ for the other method). In fact, this feature provided most of the known frequencies of such neutron stars. In addition, the amplitude and the shape of a phase-folded burst oscillation light curve can be very useful to constrain other stellar parameters. This is because the compactness $\left(G M / R c^{2}\right)$ and the spin-related surface speed $(\vartheta)$ affect these light curves through the Doppler effect, and special and general relativistic effects (including light bending). The amplitude and asymmetry of phase-folded light curves are greater for lesser $G M / R c^{2}$ values (e.g., Pechenick et al. (1983); Strohmayer et al. (1998); Miller and Lamb
(1998); Braje et al. (2000); Weinberg et al. (2001)), and hence can be used to constrain the neutron star radiusto-mass ratio. Besides, $\vartheta$ introduces a pulse phase dependent Doppler shift in the X-ray spectrum (Strohmayer and Bildsten , 2006), which can be used to estimate $\vartheta$. Note that $\vartheta$ can be useful to constrain the neutron star radius, for the known stellar spin frequency (measured from burst oscillations).

Several studies have been done to measure the neutron star properties based on the modeling of burst oscillations. These include an investigation of the amplitude and harmonic content of the phase-folded light curves due to a point-like hot spot (Miller and Lamb , 1998); and the effects of expanding single hot spot or two hot spots (Nath et al. , 2002), or single and antipodal hot spots of varying size (Weinberg et al. , 2001). Moreover, Cadeau et al. (2007) and Morsink et al. (2007) studied the effects of the oblate shape of a rapidly spinning neutron star on the phase-folded light curves.

Apart from XTE J1814-338, no other neutron star LMXB has exhibited significantly asymmetric (nonsinusoidal) phasefolded burst oscillation light curves (but see Bhattacharyya and Strohmayer (2005)). The symmetric (sinusoidal) nature of the phase-folded light curves can be attributed to the limitation of the detector capability and/or to one of the following reasons (Muno et al. , 2002b): (1) if there is one hot spot on the neutron star, it must either be close to the spinning pole or cover nearly half the stellar surface; or (2) if there are two hot spots, they must form very near the spinning equator. Information about the neutron star parameters can be extracted only from the amplitude of such a symmetric light curve, and not from its shape. Therefore, Bhattacharyya et al. (2005) fitted the nonsinusoidal burst decay oscillation light curves of XTE J1814-338 with a relativistic model appropriate for a single hot spot. This model had five input parameters for a chosen EoS model and a known neutron star spin frequency. These parameters were, (1) neutron star radiusto-mass ratio $\left(R c^{2} / G M\right)$, (2) polar angle of the center of the spot, (3) angular radius of the spot, (4) a parameter which gave a measure of the beaming in the emitter's frame, and (5) the observer's inclination angle measured from the stellar spin axis. Bhattacharyya et al. (2005) could determine a lower limit of $R c^{2} / G M$ at the $90 \%$ confidence level, in spite of the systematics due to the unknown values of the other parameters. Watts et al. (2005) and Watts and Strohmayer (2006) studied the variability and the energy dependence of the burst oscillation properties of XTE J1814-338, which may be useful to reduce these systematic uncertainties.

Since XTE J1814-338 is an accretion-powered millisecond pulsar ( $\S 3.2 .1$ ), and its modulations in the accretion and thermonuclear burst emission are phase-locked with each other (see Watts et al. (2008)), its burst decay oscillation could very well originate from a hot spot. But it is difficult to understand what gives rise to a hot spot for a non-pulsar during burst decay. Therefore, several 
groups have attempted to model burst decay oscillations of non-pulsars using an asymmetric brightness pattern more complex than a hot spot. Such a complex pattern may be caused by Rossby waves (or r-modes) in the surface layers (Lee and Strohmayer , 2005; Heyl , 2005; Piro and Bildsten , 2006), shear instabilities (Cumming, 2005), etc. Neutron star parameters (e.g., $\left.R c^{2} / G M\right)$ can, in principle, be constrained, even if burst oscillations originate from surface modes (see Lee and Strohmayer (2005); Heyl (2005)). However, in practice, it is very difficult, because one needs to identify the burst oscillations with the correct surface mode number.

Unlike burst decay oscillations of non-pulsars, the oscillations during burst rise are likely to be caused by a hot spot (e.g., Bhattacharyya and Strohmayer (2007c); Watts et al. (2009a)). This hot spot is an expanding burning region, which can be modelled more reliably than the burst decay oscillations, in order to constrain the neutron star parameters. A better understanding of thermonuclear flame spreading ( $\S 3.1 .1)$ will further increase the reliability of neutron star parameter measurement using burst rise oscillations. However note that, although an expanding hot spot is likely to cause the burst rise oscillations, this hypothesis is yet to be comprehensively tested against the entire data set.

\subsubsection{Millihertz Quasi-periodic Oscillation Method}

In 2001, Revnivtsev et al. (2001) discovered milliHertz $(\mathrm{mHz})$ period quasi-periodic oscillations (QPOs) from three neutron star LMXBs: 4U 1608-52, 4U 1636-536 and Aql X-1. This new class of timing feature has the following properties: (1) the frequency varies between $0.007-0.009$ $\mathrm{Hz}$ (period $\approx 1.9-2.4$ minutes); $(2)$ the associated flux variations are at the few percent level; (3) the strength is more at lower photon energies $(<5 \mathrm{keV}$; this is in contrast to other types of QPOs); (4) it appears in a narrow luminosity range around $10^{37} \mathrm{ergs} \mathrm{s}^{-1}$; and (5) it disappears after thermonuclear X-ray bursts. Based on the theoretical research and observational findings of Paczyński (1983); Bildsten (1993); Revnivtsev et al. (2001); Yu and van der Klis (2002); Narayan and Heyl (2003), and others, Heger et al. (2007b) proposed that the $\mathrm{mHz}$ QPOs correspond to the marginal thermonuclear burning stability on the neutron star surfaces close to the bounday between regime 3 and regime 4 mentioned in $\S$ 3.1.1. Near such a bounday, oscillations are expected because the eigenvalues of the system are complex (Paczyński , 1983; Narayan and Heyl , 2003). The narrow range of accretion rate corresponding to the bounday can explain why $\mathrm{mHz}$ QPOs appear in a narrow luminosity range. Heger et al. (2007b) found that the period of the oscillations due to the marginal burning stability should be $\approx \sqrt{t_{\text {thermal }} t_{\text {accretion }}}(\approx 2$ min $)$, where $t_{\text {thermal }}$ is the thermal timescale of the burning layer and $t_{\text {accretion }}$ is the time to accrete the fuel at the Eddington accretion rate. This theoretical period matches well with the observed period, which is the main strength of the Heger et al. (2007b) model. However, note that a few aspects of $\mathrm{mHz}$ QPOs are still not understood (Heger et al. , 2007b).

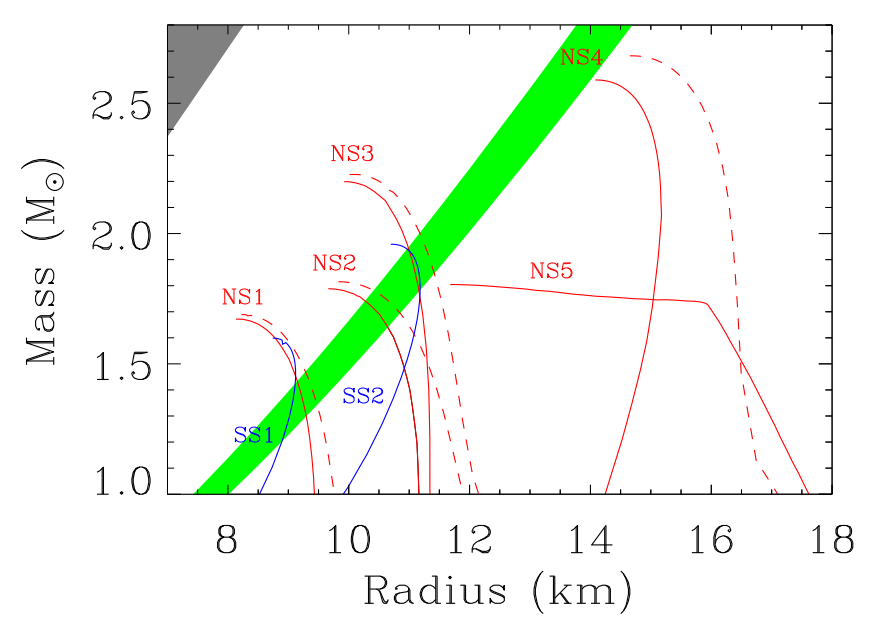

Figure 10: This figure shows the $M-R$ space of neutron stars with the curves corresponding to a few representative EoS models (same as Figure 1). The green patch shows the allowed $M-R$ space using equation 7 for a reasonable range of the surface gravity $(g=[2.6-$ $\left.3.1] \times 10^{14} \mathrm{~cm} \mathrm{~s}^{-2}\right)$. This example figure demonstrates how the measured surface gravity can be used to constrain the neutron star $M-R$ space (see $\S 3.1 .7)$.

The theoretically proposed oscillation frequency is a strong function of the surface gravity and the chemical composition of the accreted matter (see Figure 9 of Heger et al. (2007b)). Therefore, if the Heger et al. (2007b) model is correct, and if the chemical composition can be determined (see $\S 3.1 .3$ for a discussion of plausible methods), then $\mathrm{mHz}$ QPOs can be used to constrain the $M-R$ space of neutron stars (see Figure 10). This is because the surface gravity $g$ is a function of stellar mass and radius

$$
g \simeq G M / R^{2}\left[1-2 G M / R c^{2}\right]^{-1 / 2} .
$$

Here we note that the marginal burning stability depends on the stellar spin rate (Keek et al. , 2009), and hence the neutron star spin can also affect the $\mathrm{mHz}$ QPOs. However, fortunately the stellar spin frequencies of all the currently known $\mathrm{mHz}$ QPO sources have meen measured (Lamb and Boutloukos , 2008).

$\mathrm{Yu}$ and van der Klis (2002) found that kilohertz QPO (see $\S 3.3$ ) frequency is anticorrelated with the luminosity when $\mathrm{mHz}$ QPOs are observed. This is opposite to the long-term trend. Therefore, $\mathrm{mHz}$ QPOs affect the kilohertz QPOs, and hence can be useful to constrain the models of the latter. Such a constraint can be very helpful to measure the neutron star parameters (see $\S 3.3 .2 ; \oint 3.4 .2$ ). Altamirano et al. (2008) found that $\mathrm{mHz}$ QPOs can also be used to predict the occurrence of thermonuclear bursts. Such a prediction may be helpful to observe more bursts, as the duty cycle of bursts is small. 


\subsubsection{Joint Application of the Methods based on Ther- monuclear X-ray Bursts}

Thermonuclear X-ray bursts provide various methods to measure the neutron star parameters $(\S 3.1 .3 ; \S 3.1 .4$; $\S 3.1 .5 ; \S 3.1 .6 ; \S 3.1 .7)$. Figures 4,6 and 8 show how some of these methods can be used to constrain the neutron star $M-R$ space. Several of the burst properties, which provide these methods, can be observed from a given neutron star LMXB. For example, apart from the surface spectral lines, all these burst features are observed from 4U 1636-536. Similarly, all the burst features, except $\mathrm{mHz}$ QPOs, have possibly been observed from EXO 0748-676. Therefore, joint application of the burst methods can be very useful to constrain the parameters of the same neutron star. For example, the continuum spectrum method ( $\S 3.1 .3)$, the spectral line method $(\S 3.1 .4)$ and the PRE burst method ( $\S 3.1 .5)$ together may constrain the $M-R$ space effectively (e.g., the intersection of the green patches of Figures 4, 6 and 8; see also Figure 1 of Özel (2006)). The first and the third of the above methods, and the known source distance, can also reduce the systematic uncertainties (e.g., Özel et al. (2009)). Moreover, Bhattacharyya and Strohmayer (2007a) have demonstrated that the joint application of the continuum spectrum method and the burst oscillation method (§ 3.1.6) can constrain the neutron star parameters by eliminating the source distance parameter.

A given neutron star LMXB can exhibit many bursts with various properties (e.g., oscillations, photospheric radius expansion, etc.). These bursts may be observed with different satellites (e.g., RXTE; XMM-Newton) at different times. The burst properties depend on neutron star and other system parameters. Neutron star global parameter values remain unaltered for all these observations, although some other source parameter values (e.g., the hot spot size for oscillations) are expected to change. Here we briefly describe how these observations can be combined within the framework of Bayesian statistics, in order to constrain the neutron star parameters effectively (see Bhattacharyya et al. (2005)). Let the posterior probability density (which we determine by fitting the data with a model) over the full set of model parameters $x_{1} \ldots x_{n}$ be $p\left(x_{1} \ldots x_{n}\right)$. If we are interested in the confidence regions for a single parameter $x_{k}$, then we integrate this probability distribution over the "nuisance" parameters $x_{1} \ldots x_{k-1}$ and $x_{k+1} \ldots x_{n}$ :

$$
q\left(x_{k}\right)=\int \mathrm{d} x_{1} \ldots \mathrm{d} x_{k-1} \mathrm{~d} x_{k+1} \ldots \mathrm{d} x_{n} p\left(x_{1} \ldots x_{n}\right) .
$$

The maximum likelihood of $x_{k}$, as well as its confidence regions, can be obtained from the probability function $q\left(x_{k}\right)$. Using this method, one can obtain a tight joint constraint on a neutron star global parameter (e.g., mass, radius, etc.) which does not vary from one observation to another, by integrating over the nuisance parameters of all the observations.

\subsection{Accretion-powered Millisecond Period Pulsations}

\subsubsection{What is an Accretion-powered Millisecond Pulsar?}

Several neutron star LMXBs exhibit coherent fast (period $\sim 1$ millisecond) brightness pulsations in their persistent X-ray emissions (e.g., non-burst, non-quiescent, etc.; $\S 3.1 .1$; $\S 3.5 .1$; wijnands (2005); Poutanen (2008); Altamirano et al. (2009a) and references therein). These pulsations can be detected as a narrow peak in the power spectrum. In these sources, the accretion flow is channeled by the neutron star magnetic field to the magnetic poles. The X-ray hot spots on the neutron star created by this channeled flow rotate around the stellar spin axis (if the magnetic axis and the spin axis are misaligned) and give rise to the observed X-ray pulses. Note that one of the hot spots is usually obsecured by the accretion disk. Since the gravitational energy release from the accreted matter powers the X-ray pulsations, these sources are called "accretion-powered millisecond pulsars (AMPs)".

In 1998, the first AMP SAX J1808.4-3658 was discovered with the $R X T E$ satellite (Wijnands and van der Klis , 1998a; Chakrabarty and Morgan , 1998). This AMP exhibits significant pulsations at $\approx 401 \mathrm{~Hz}$. The data revealed that (1) the low-energy pulses lag the high-energy pulses, and (2) the pulse profiles are significantly asymmetric (nonsinusoidal) allowing a detection of even the second harmonic (Cui et al. , 1998). Poutanen and Gierliński (2003) suggested that both these properties could be explained in terms of two spectral components: a 'pencil'-like beamed blackbody emission and a 'fan'-like Comptonized emission (from a radiative shock; but see also Hartman et al. (2009)).

Four years after the discovery of the first AMP, the second AMP XTE J1751-305 was reported by Markwardt et al. (2002). Since then ten more AMPs have been discovered (wijnands (2005); Altamirano et al. (2009a,b); Markwardt et al (2009); and references therein). All these AMPs, including the first two, are transient sources (pulsations are detected only during the outbursts; $\S 3.5 .1$; Lamb et al. (2008a)), and three of them show pulsations very occasionally (see Watts et al. (2009a) and references therein). Here we do not describe the properties of these AMPs in detail, and suggest the following references for the interested readers: Gilfanov et al. (1998); Psaltis and Chakrabarty (1999); Bildsten and Chakrabarty (2001); Miller et al. (2003); Nelson and Rappaport (2003); wijnands (2005); van Straaten et al. (2005); Poutanen and Beloborodov (2006); van der Klis (2006); Watts et al. (2008); Poutanen (2008); Cackett et al. (2009a); etc. Note that, although many properties of AMPs are broadly understood, it is still a matter of debate why only a few neutron star LMXBs are AMPs (e.g., Titarchuk et al. (2002); Lamb et al. (2008a); Özel (2009)). Study of the intermittent pulsation sources, among other things, may be useful to solve this problem (e.g., Lamb et al. (2008b); Watts et al. (2009a)). 


\subsubsection{Accretion-powered Millisecond Pulsation Method}

Accretion-powered pulsation frequency is the neutron star spin frequency, because such pulsations are caused by the periodic motion of a hot spot due to the stellar spin (see 3.2.1). These pulsations are more coherent than burst oscillations ( $\S 3.1 .6$ ), and, unlike the burst oscillation frequency, the pulsation frequency does not evolve appreciably in a short time. Therefore, the pulsation method gives a more accurate measurement of the spin frequency.

The phase-folded accretion-powered pulsation light curves are affected by the Doppler effect, and the special and general relativistic effects. Therefore, similar to the burst oscillations, the amplitude and the shape of a pulsation light curve can also be very useful to measure the neutron star and other system papameters (see $\S 3.1 .6$ for the burst oscillation method). Various groups attempted to constrain the stellar parameters by fitting the pulsation light curves with appropriate relativistic models (e.g., Poutanen and Gierliński (2003, 2004); Leahy et al. (2008, 2009)). However, similar to the burst oscillation method (see $\S 3.1 .6$ ), the systematic uncertainties due to the unknown system parameters (such as, observer's inclination angle, size and polar angle of the hot spot, etc.) hinder an accurate measurement. Besides, additional systematics due to the uncertainties in the spectral properties of various X-ray emitting components make the pulsation method less reliable (see the next paragraph).

Both burst oscillations and accretion-powered pulsations originate from azimuthally asymmetric brightness patterns on the spinning neutron star surfaces. Therefore, in principle, similar methods can be used to extract information from them. However, in practice, these two methods are somewhat different from each other because of the following reasons.

(1) Phase-folded burst oscillation light curves appear symmetric or sinusoidal with the current observational capabilities (see, Strohmayer et al. (2003) and Bhattacharyya and Strohmayer (2005) for exceptions). However, the accretion-powered pulsation light curves can be nonsinusoidal, especially, but not exclusively, at higher energies (e.g., Cui et al. (1998); Poutanen (2008)). Therefore, in principle, more information can be extracted from the accretion-powered pulsation light curves (using both amplitudes and shapes; see $\S 3.1 .6$ ).

(2) During a thermonuclear burst, most of the emission originates from the neutron star surface, and the corresponding energy spectrum can be fitted well with a blackbody model (see $\S$ 3.1.3). Moreover, only burst emission contributes to the oscillations, and the gravitational energy release due to the accretion can be separated from this burst emission (see Galloway et al. (2008a)). These make the modeling of phase-folded burst oscillation light curves simple and reliable. But during accretion-powered pulsations, gravitational potential energy powers both the disk and the surface emissions. Moreover, the surface emission, which gives rise to the pulsations, is at least partially processed (Comptonized) in the bulk motion of matter (e.g., Mastichiadis and Kylafis (1992)) channelled by the stellar magnetic field, and/or in a radiative shock at the neutron star surface (Poutanen and Gierliński , 2003). It is very difficult to observationally separate these various components of emission, as well as their contributions to the phase-folded pulsation light curves, from each other. This makes the understanding of pulsation light curves difficult, and reduces the reliability of the pulsation method. Rigorous theoretical computation of both time-averaged and phase-resolved energy spectra is required to solve this problem. For example, among other things, one may need to perform the Monte Carlo simulation of the scattering of photons in the bulk electron flow near the magnetic poles, including the gravitational redshift and light bending effects.

(3) Unlike burst oscillations, accretion-powered pulsations can be useful to test the effects of accretion rate changes on the inner-edge radius of the accretion disk, the size of the hot spot, etc. (Ibragimov and Poutanen , 2009). This can, in turn, be useful to estimate a few system parameters (e.g., neutron star magnetic field; Ibragimov and Poutanen (2009)), and hence to make some of the methods described throughout this review more reliable.

Finally, we note that other methods have been proposed in the literature to constrain the neutron star $M-R$ space for AMPs (Bhattacharyya , 2001). Gilfanov et al. (1998) reported that the X-ray energy spectrum of SAX J1808.4-3658 had remained stable, when the X-ray luminosity varied by two orders of magnitude. Bhattacharyya (2001) (see also Burderi and King (1998); Li et al. (1999)) found the upper limits to the mass and the radius of the neutron star using this observational result, and with the following assumptions: (1) the neutron star magnetic field is dipolar; (2) there is no "intrinsic" pulse mechanism; and (3) the accretion flow is not centrifugally inhibited.

\subsection{Kilohertz Quasi-periodic Oscillations}

\subsubsection{What are Kilohertz Quasi-periodic Oscillations?}

Neutron star LMXBs exhibit a zoo of timing features, both broad and narrow, in the Fourier power spectra (see van der Klis (2006) for an excellent review). Many of these features are correlated with each other and with the spectral states of the source (van der Klis (2006) and references therein). Among these features, high frequency quasi-periodic oscillations, or kilohertz $(\mathrm{kHz})$ QPOs, are the most important ones to constrain the neutron star parameters (see $\S 3.3 .2$ ). These QPOs often occur in a pair (Figure 11), and the twin peaks usually move together in the frequency range $\sim 200-1200 \mathrm{~Hz}$ in correlation with the source state. The higher frequency QPO is known as the upper $\mathrm{kHz}$ QPO (frequency $=\nu_{u}$ ), and the lower frequency $\mathrm{QPO}$ is called the lower $\mathrm{kHz} \mathrm{QPO}$ (frequency $\left.=\nu_{l}\right)$. The $\mathrm{kHz}$ QPOs were first reported in van der Klis et al. (1996) and Strohmayer et al. (1996) (see also van der Klis (1998) for a historical account). As of now 
these QPOs have been observed from about 30 neutron star LMXBs (see Altamirano et al. (2009a); van der Klis $(2006))$.

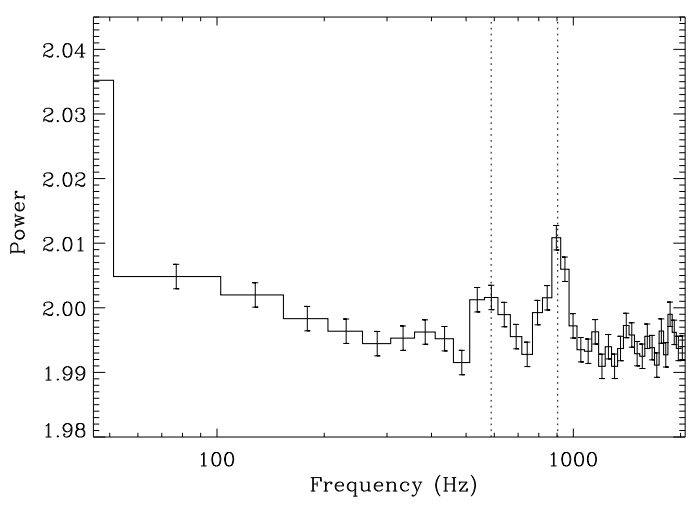

Figure 11: Power spectrum of a neutron star LMXB showing a pair of $\mathrm{kHz}$ QPOs (see $\S$ 3.3.1). The vertical dotted lines show the centroid frequencies of these QPOs.

Although frequency $(\nu)$, amplitude, quality factor $(\nu / \delta \nu)$ and other properties of $\mathrm{kHz}$ QPOs vary from source to source, and even for a given source depending on its intensity and spectral state, several general trends of this timing feature could still be established. Here we mention some of them which may be useful to understand these QPOs. (1) For a given source, $\nu$ correlates well with the X-ray luminosity $L_{x}$ on time scales of hours. But on longer time scales, and across sources, the $\nu-L_{x}$ correlation does not exist, and similar $\nu$ values are observed over two orders of magnitude in $L_{x}$ (e.g., van der Klis (1997)). (2) It was thought that the frequency separation $\nu_{u}-\nu_{l}(=\Delta \nu)$ clusters around the neutron star spin frequency and the half of it (Wijnands et al. (2003); van der Klis (2006) and references therein); although the recent works of Méndez and Belloni (2007) and others have suggested that $\Delta \nu$ is consistent with no dependence on spin. (3) KHz QPO amplitudes increase with photon energy, and in similar energy bands, the QPOs are weaker in the more luminous sources (Jonker et al. (2001); van der Klis (2006) and references therein). (4) The quality factor of lower $\mathrm{kHz}$ QPOs increases with the QPO frequency at lower frequency values, and decreases at higher frequency values. But the quality factor of upper $\mathrm{kHz}$ QPOs increases with the frequency all the way to the highest detectable frequencies (Barret et al. , 2006). (5) KHz QPO frequencies are correlated with the frequencies (or characteristic frequencies) of many narrow and broad timing features from neutron star LMXBs (see Figure 2.9 of van der Klis (2006)).

What causes the $\mathrm{kHz}$ QPOs is not known yet, although many models are available in the literature. A discussion of all these models is out of the scope of this review. Here, we will mention only a few models which will be used in $\S 3.3 .2$ to demonstrate the plausible ways to measure neutron star parameters using $\mathrm{kHz}$ QPOs. The most promi- nent property of a $\mathrm{kHz}$ QPO is its high frequency. Most of the models have primarily attempted to explain this property. This frequency is usually believed to be caused by the frequencies related to the fast motions close to a neutron star, where the effects of general relativity is important. Let us first identify some of these frequencies, and write down their expressions for circular orbits in the equatorial plane for Kerr spacetime.

(1) Orbital frequency around the neutron star

$$
\nu_{\phi}=\nu_{\mathrm{K}}\left(1+j\left(r_{g} / r\right)^{3 / 2}\right)^{-1}
$$

where $\nu_{\mathrm{K}}=\sqrt{G M / r^{3}} / 2 \pi$. Here, $r$ is the radial distance from the center of the neutron star, $r_{g} \equiv G M / c^{2}$, and the angular momentum parameter $j \equiv J c / G M^{2}$, where $J$ is the stellar angular momentum.

(2) Radial epicyclic frequency (for infinitesimally eccentric orbits)

$$
\nu_{r}=\nu_{\phi}\left(1-6\left(r_{g} / r\right)+8 j\left(r_{g} / r\right)^{3 / 2}-3 j^{2}\left(r_{g} / r\right)^{2}\right)^{1 / 2} .
$$

(3) Vertical epicyclic frequency (for infinitesimally tilted orbits)

$$
\nu_{\theta}=\nu_{\phi}\left(1-4 j\left(r_{g} / r\right)^{3 / 2}+3 j^{2}\left(r_{g} / r\right)^{2}\right)^{1 / 2} .
$$

(4) Periastron precession frequency $\nu_{\text {peri }}=\nu_{\phi}-\nu_{r}$.

(5) Nodal precession frequency $\nu_{\text {nodal }}=\nu_{\phi}-\nu_{\theta}$.

Apart from these, the neutron star spin frequency $\nu_{\text {spin }}$ may also contribute to $\mathrm{kHz}$ QPOs. The radial profiles of the general relativistic frequencies are shown in the Figure 12. This figure helps to understand any model based on these frequencies. It also clearly shows that the radial epicyclic frequency cannot be $\nu_{u}$ or $\nu_{l}$, because some $\mathrm{kHz}$ QPOs are observed with frequencies much higher than the maximum possible value of $\nu_{r}$. In many models, $\mathrm{kHz}$ QPO frequencies are explained in terms of the above mentioned general relativistic frequencies and $\nu_{\text {spin }}$ (or beating between any two of them) at some preferred radii. A preferred radius may be determined by the disk flow structure (depends on, for example, the accretion rate), the radius of the general relativistic innermost stable circular orbit (ISCO), or the commensurabilities among the frequencies which may cause resonance. For example, Miller et al. (1998) identified $\nu_{u}$ and $\nu_{l}$ with $\nu_{\phi}$ and a beating frequency $\left(\nu_{\text {beat }}\right)$ respectively at the "sonic" radius $\left(r_{\text {sonic }}\right)$. Lamb and Miller (2003) modified this model maintaining $\nu_{u}=\nu_{\phi}\left(r_{\text {sonic }}\right)$, and explaining $\nu_{l}$ by a beat-frequency interaction between orbital motion at $r_{\text {sonic }}$ and an azimuthal structure at the spin-resonance radius, defined by $\nu_{\text {spin }}-\nu_{\phi}=\nu_{\theta}$. Stella and Vietri $(1998,1999)$ identified $\nu_{u}$ with $\nu_{\phi}$ at the disk inner edge, and related $\nu_{l}$ and $\nu_{h}$ (frequency of a low-frequency QPO; see van der Klis (2006)) with $\nu_{\text {peri }}$ and $\nu_{\text {nodal }}$ respectively. Various authors (e.g., Kluźniak and Abramowicz (2001); Abramowicz and Kluźniak (2001)) made use of the fact that $\nu_{\phi}, \nu_{r}$ and $\nu_{\theta}$ at particular radii have simple integer ratios or other commensurabilities with each other or with $\nu_{\text {spin }}$. They suggested that at these radii resonances may occur which can 
show up as $\mathrm{kHz}$ QPOs. Besides, many other authors have used the general relativistic frequencies in various ways to explain the properties of $\mathrm{kHz}$ QPOs (e.g., Wijnands et al. (2003); Lee et al. (2004); Zhang (2004); Mukhopadhyay (2009)).

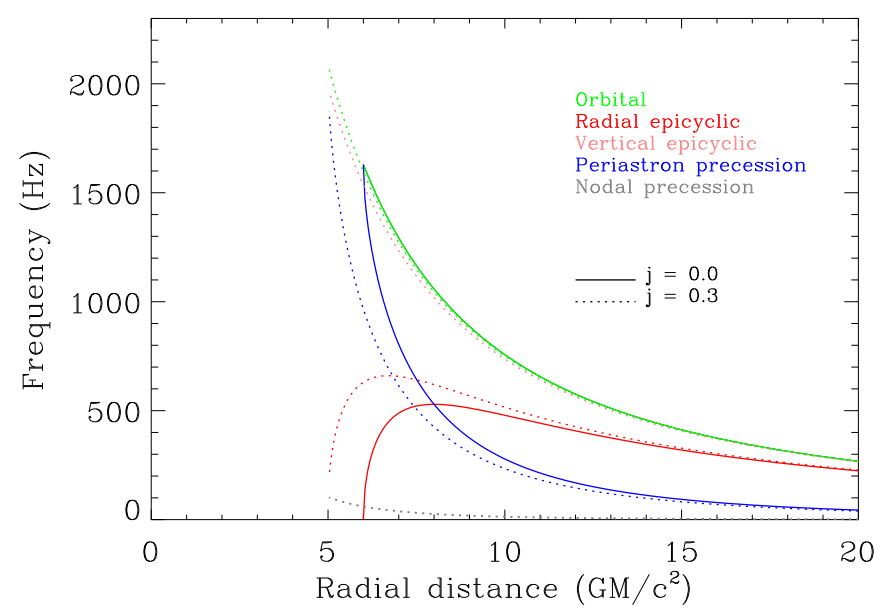

Figure 12: This figure shows the radial profiles of various frequencies (color coded) of equatorial circular orbits in Kerr spacetime (see $\S 3.3 .1)$. Two angular momentum parameters $(j=0.0$ (solid) and $j=0.3$ (dotted) , and neutron star mass $=1.35 M_{\odot}$ are used. These frequencies may be useful to understand the $\mathrm{kHz}$ QPOs (see $\S 3.3 .1$ ).

Apart from the observed frequencies of $\mathrm{kHz}$ QPOs, one also needs to explain the modulation and decoherence mechanisms. Some of the plausible methods have been discussed in van der Klis (2006) and references therein. Méndez (2006) proposed that, although the $\mathrm{kHz}$ QPO frequencies are plausibly determined by the characteristic disk frequencies (see the previous paragraph), the modulation mechanism is likely associated to the high energy spectral component (e.g., accretion disk corona, boundary layer between the disk and the neutron star, etc.). This is because the disk alone cannot explain the large observed amplitudes, especially at hard X-rays where the contribution of the disk is small. Detection and measurement $\mathrm{kHz}$ QPOs above $20-30 \mathrm{keV}$ may be able to resolve this issue. In addition, observations of sidebands, overtones, and very high frequency QPOs may be useful to identify the correct kHz QPO model (van der Klis , 2006; Bhattacharyya , 2009). We will not discuss these aspects further. Rather we note that the fluid dynamical simulation corresponding to any successful $\mathrm{kHz}$ QPO model must naturally give rise to the observed frequencies and other properties.

\subsubsection{Kilohertz Quasi-periodic Oscillation Method}

Since no kHz QPO model can yet explain all the major aspects of this timing feature, currently it is not possible to constrain the neutron star parameters using this QPOs with certainty. However, all proposed models in- volve plasma motion in the strong gravitational field around the neutron star, and with one exception (photon bubbles; Klein et al. (1996a,b)) suggest that the $\mathrm{kHz}$ QPOs originate in the disk (van der Klis , 2006). Moreover, most models identify one of the $\mathrm{kHz}$ QPO frequencies (usually $\nu_{u}$; but can also be $\nu_{l}$ ) with the orbital motion at a preferred disk radius (van der Klis (2006); see also $§ 3.3 .1$ ). If this is true, two reasonable conditions can constrain the $M-R$ space (Miller et al. , 1998):

$$
R \leq r
$$

where $r$ is the radius of the orbit associated with $\nu_{u}$ or $\nu_{l}$ via equation 9 ; and

$$
r_{\mathrm{ISCO}} \leq r,
$$

where $r_{\text {ISCO }}$ is the radius of the ISCO. This is because the first condition gives a mass-dependent upper limit on $R$ via equation 9 ; and the second condition gives an upper limit on $M: \quad M<c^{3} /\left(\left.2 \pi 6^{3 / 2} G \nu_{\phi}\right|_{r}\right.$ ) (for Schwarzschild spacetime). These constraints on $M-R$ space are shown in Figure 13.

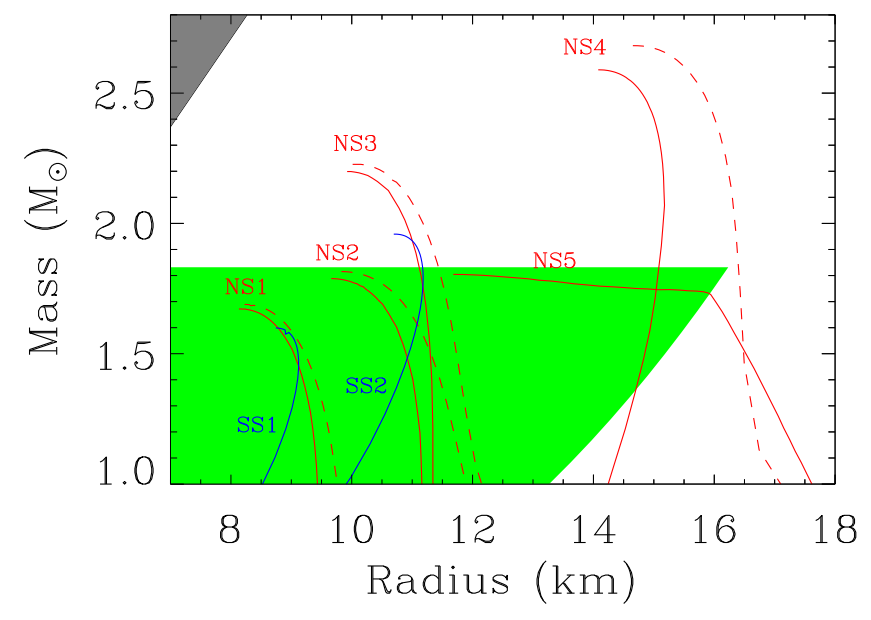

Figure 13: This figure shows the $M-R$ space of neutron stars with the curves corresponding to a few representative EoS models (same as Figure 1). The green patch shows the allowed $M-R$ space using equations 12 and 13, and an upper $\mathrm{kHz}$ QPO frequency of $1200 \mathrm{~Hz}$ (Miller et al. (1998); see $\S 3.3 .2$ ). This example figure demonstrates the potential of $\mathrm{kHz}$ QPOs to constrain the neutron star mass and radius, and hence the EoS models.

The general relativistic frequencies depend on the neutron star parameters $M$ and $j$, and many models use these frequencies to explain $\mathrm{kHz}$ QPOs ( $\S 3.3 .1$; see also van der Klis (2006) and references therein). Therefore, in a more general sense the identification of any of the $\mathrm{kHz}$ QPO frequencies with the beating, resonance, or any other combination of $\nu_{\phi}, \nu_{r}, \nu_{\theta}, \nu_{\text {peri }}, \nu_{\text {nodal }}$ and $\nu_{\text {spin }}$ has the potential to constrain the neutron star parameter space. Besides, the identification of $\nu_{h}$ with $\nu_{\text {nodal }}(\S 3.3 .1$ ) can be 
useful to constrain the stellar moment of inertia $I$, because $\nu_{\text {nodal }}=8 \pi^{2} \nu_{\phi}^{2} I \nu_{\text {spin }} / M c^{2}$ (Stella and Vietri , 1998). Finally, simultaneous observations of $\mathrm{kHz}$ QPOs and broad relativistic iron lines have the potential to measure neutron star parameters (see $\S 3.4 .2$ ).

\subsection{Broad Relativistic Iron Lines}

\subsubsection{What is a Broad Relativistic Iron Line?}

A broad iron $\mathrm{K} \alpha$ spectral emission line near $6 \mathrm{keV}$ is observed from many accreting supermassive and stellar-mass black hole systems (see Fabian et al. (2000); Reynolds and Nowak (2003); Miller (2007); and references therein). Although various models were proposed to explain this feature (e.g., Misra and Kembhavi (1998); Titarchuk et al. (2003); Reeves et al (2004); Titarchuk et al. (2009)), the large width, the lower-energy wing and the asymmetry of such a line, as well as the observed associated "disk reflection" spectrum strongly suggest that this line originates from the inner part of the accretion disk, where it is shaped by Doppler and relativistic effects (e.g., Tanaka et al. (1995); Fabian et al. (2000); Miller et al. (2001); Miniutti et al. (2007); Miller et al (2009)). This relativistic line are believed to be produced by the reflection of hard X-rays from the accretion disk. The hard X-ray source may be anything from an accretion disk corona (Fabian et al. , 2000) to the base of a jet (Markoff and Nowak , 2004; Markoff et al. , 2005). A given incident X-ray photon may be Compton scattered by free or bound electrons, or subject to photoelectric absorption followed by either Auger de-excitation or fluorescent line emission (Fabian et al. , 2000). The strongest among the fluorescent spectral emission lines is the one for the $n=2 \rightarrow n=1$ transition of the iron atom (or ion). This iron $\mathrm{K} \alpha$ line originates at an energy between $6.4 \mathrm{keV}$ and $6.97 \mathrm{keV}$, depending on the iron ionization state (Reynolds and Nowak, 2003). The intrinsically narrow iron $\mathrm{K} \alpha$ line is shaped by the following effects: (1) the Doppler effect due to the motion of the line-emitting matter in the inner part of the accretion disk broadens the line and makes it double-peaked; (2) The general relativistic light-bending effect also contributes to the formation of the two peaks; (3) the transverse Doppler shift and the gravitational redshift shift the line towards lower energies; and (4) the special relativistic beaming reduces the lower-energy peak and enhances the higher-energy peak making the line asymmetric (we encourage the readers to see the Figure 3 of Fabian et al. (2000)). Therefore, the relativistic iron line provides an ideal tool to probe the spacetime and the flow and structure of accreted matter in the strong gravity region. The modelling of such a line can also be useful to constrain the radius of the ISCO (in the unit of black hole mass), which can be used to measure the black hole spin parameter or angular momentum parameter $\left(j \equiv J c / G M^{2} \equiv a / M ; J\right.$ and $M$ are the black hole angular momentum and mass respectively; see, for example, Brenneman Reynolds (2006); Miller et al (2009)). This is because $a / M$ has a one-toone correspondence with the ratio of the ISCO radius to black hole mass for Kerr spacetime (see Figure 1a of Miller (2007)).

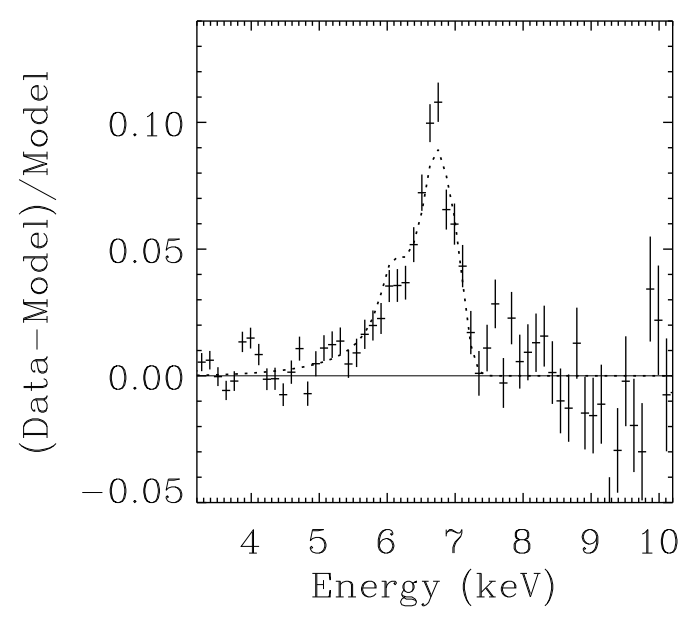

Figure 14: Broad relativistic iron $\mathrm{K} \alpha$ spectral line from the inner accretion disk of a neutron star LMXB Serpens X-1 (Bhattacharyya and Strohmayer , 2007b). The x-axis shows the X-ray energy, and the $\mathrm{y}$-axis gives the observed intensity in excess to the best-fit continuum spectral model. The data points clearly show a broad asymmetric spectral emission line, while the dotted profile is a relativistic model which fits the observed line well (§ 3.4).

Similar to black hole systems, a broad iron line from neutron star LMXBs has also been known for many years (see, for example, Asai et al. (2000)). However, the inner accretion disk origin of this line could not be established until recently. This was because the characteristic asymmetry of the relativistic nature could not be detected due to the modest signal-to-noise ratio. In 2007, Bhattacharyya and Strohmayer (2007b), for the first time, established the inner accretion disk origin of the broad iron line from a neutron star LMXB (see Figure 14). This was done by analyzing the XMM-Newton data from Serpens $\mathrm{X}-1$. Soon, Cackett et al. (2008a) confirmed this finding using the independent Suzaku data from the same source. These authors also reported the detection of relativistic iron lines from two other neutron star LMXBs: 4U 182030 and GX $349+2$. As of now, the inner disk origin of broad iron line has been confirmed for ten neutron star LMXBs (Bhattacharyya and Strohmayer, 2007b; Cackett et al. , 2008a; Pandel et al. , 2008; D'Aí et al. , 2009; Cackett et al. , 2009a; Papitto et al. , 2009; Shaposhnikov et al. , 2009; Reis et al. , 2009; di Salvo et al. , 2009; Iaria et al. , 2009; Cackett et al. , 2009b). This opens up a new way to measure neutron star parameters, as described in $\S 3.4 .2$.

\subsubsection{Broad Relativistic Iron Line Method}

The broad relativistic iron line can be used to constrain various neutron star parameters. This line originates from an annular region of the inner accretion disk (see $\S 3.4 .1$ ). 
The ratio of the inner edge radius of this annulus $\left(r_{\mathrm{in}, \mathrm{ann}}\right)$ to the neutron star mass $(M)$ can be constrained from the fitting of the iron line with a relativistic model (see, for example, Bhattacharyya and Strohmayer (2007b)). Since the disk inner edge radius has to be obviously greater than or equal to the neutron star radius, the measured $r_{\text {in, ann }} c^{2} / G M$ gives a hard upper limit on the neutron star $R c^{2} / G M$. This can be useful to constrain the stellar $M-R$ space (see, for example, Figure 6).

The relativistic iron line may also be used to constrain the neutron star angular momentum parameter, if $r_{\mathrm{in}, \text { ann }} c^{2} / G$ $<6$. Here is how. The accretion disk can extend up to the radius $\left(r_{\mathrm{ISCO}}\right)$ of the ISCO, or the neutron star radius $(R)$, whichever is greater (see Figure 1 of Bhattacharyya et al. (2000)). However, the disk may be truncated at a larger radius by the magnetic field and/or radiative pressure, and/or by any other means. Therefore, if $r_{\text {in }}$ is the disk inner edge radius, then $r_{\text {in, ann }} c^{2} / G M \geq r_{\text {in }} c^{2} / G M \geq$ $R c^{2} / G M$ and $r_{\text {in, ann }} c^{2} / G M \geq r_{\text {in }} c^{2} / G M \geq r_{\mathrm{ISCO}} c^{2} / G M$. For a non-spinning neutron star, $r_{\mathrm{ISCO}} c^{2} / G M=6$, and for a spinning neutron star with a corotating disk, $r_{\mathrm{ISCO}} c^{2} / G M$ $<6$. Therefore, if the best fit value of $r_{\text {in,ann }} c^{2} / G M$ is less than 6 , then that must be because of the effect of the stellar spin on the spacetime, and hence can, in principle, be useful to constrain the neutron star angular momentum parameter (see Figure 1a of Miller (2007)). Here we note that the Kerr spacetime has been assumed in the Figure 1a of Miller (2007), while the exterior spacetime of a spinning neutron star deviates from the Kerr geometry (Miller and Lamb , 1996).

In $\S 3.3$, we have mentioned that $\mathrm{kHz}$ QPOs are likely associated with the inner accretion disk. If this is true, then the relativistic iron line and the $\mathrm{kHz}$ QPOs are possibly physically related, and their simultaneous observation and joint analysis may, not only shed light on the physics of these features, but also be useful to constrain the neutron star parameters. For example, Miller et al. (1998) suggested that the upper $\mathrm{kHz}$ QPO frequency $\left(\nu_{\mathrm{u}}\right)$ is close to the orbital frequency at the inner edge of the optically thick emission, and thus from a radius close to the $r_{\text {in, ann }}$ inferred from the iron line. If this is true, then the neutron star mass $(M)$ can be measured from the relation $\nu_{\mathrm{u}}=(1 / 2 \pi) \sqrt{G M / r_{\text {in,ann }}^{3}}$ (assuming Schwarzschild spacetime; Cackett et al. (2008a)).

Broad relativistic iron line method has emerged as a way to constrain the neutron star parameters only recently. Although this method has a great potential, it suffers from systematics similar to other methods. These systematic uncertainties may appear due to (1) the lack of knowledge of various parameters, such as the observer's inclination angle, the nature of the hard X-ray emission incident upon the disk, etc; and (2) the inability to model the continuum spectrum accurately. Here we will not discuss these uncertainties in detail. Rather, we will only note that the incident hard X-ray emission in the neutron star LMXBs is currently believed to be originated from the boundary layer between the inner accretion disk and the neutron star surface (Cackett et al. , 2009b), although more studies are required to verify this.

\subsection{Quiescent Emissions}

\subsubsection{What is Quiescent Emission?}

Many neutron star LMXBs have two distinctly different intensity states: (1) the quiescent state in which the source remains for months to years; and (2) the outburst state which continues for weeks to months (sometimes for Years; e.g., for the source KS 1731-260). These LMXBs are called transients. The luminosity of the source typically increases by several orders of magnitude as it evolves from the quiescent state into an outburst. Figure 1 of Bhattacharyya et al. (2006c) shows the long term intensity profile of such a transient 1A 1744-361, exhibiting three outbursts. The transient nature of these LMXBs is normally attributed to an accretion disk instability, which causes high accretion rates for certain periods of time (outbursts), and almost no accretion during other times (quiescence; see King (2001) for a review). Therefore during the quiescent state, the observed X-ray emission is expected to primarily originate from the neutron star surface. This emission can be detected and measured with high sensitivity imaging instruments on board Chandra, XMM-Newton, etc. (e.g., Wijnands et al. (2002); Cackett et al. (2008b); Heinke et al. (2009)).

\subsubsection{Quiescent Emission Method}

As mentioned in $\S 3.5 .1$, the emission from a transient neutron star LMXB in quiescence primarily comes from the neutron star's atmosphere. This atmosphere should be composed of pure hydrogen, and devoid of any X-ray spectral line (see, for example, van Kerkwijk (2004)). Moreover, the relatively low magnetic fields of neutron stars in LMXBs (unlike isolated neutron stars, and stars in double neutron star binaries and HMXBs) make the modeling simpler. Therefore, assuming a blackbody emission from the neutron star surface in a quiescent LMXB, one can constrain the stellar radius using equations 2 and 4 . As mentioned in $\S 3.1 .3$, there may be effects of systematics in the estimated radius. During quiescence, the entire neutron star surface is expected to emit uniformly, because the stellar magnetic field is low, and there is no obvious effect which can create a significant asymmetry. Therefore, The systematic uncertainty \#1 ( $\S 3.1 .3$ ) may not be present. The source distance, which appears in equation 2 , may be known from a PRE burst from the source, or in case the source is in a globular cluster (see uncertainty \#2; $\S 3.1 .3$ ). The surface gravitational redshift $1+z$, which appears in equation 4 and causes the uncertainty \#3 (§3.1.3), may be determined from an independent measurement of the neutron star radius-to-mass ratio (see, for example, $\S 3.1 .4$; $\S 3.1 .6 ; \oint 3.4 .2)$. But, even if this ratio is not known, the neutron star $M-R$ space can still be constrained as shown in Figure 4. The color factor $f$, which appears in 
equation 4 and causes the uncertainty \#4 (§ 3.1.3), may be determined by theoretical calculations. However, if a theoretical atmospheric spectrum for hydrogen is used for fitting (instead of a blackbody spectrum), then a separate computation of $f$ is not required. In fact, hydrogen atmospheric spectrum is usually used to model the quiescent emission, which can constrain the inferred radius $R_{\infty}$ of the neutron star well (equations $2 \& 4$; Gendre et al. (2003); Webb and Barret (2007); Guillot et al. (2009)).

Apart from the above systematics, three observational aspects make the quiescent emission method less reliable. (1) The interstellar absorption cuts off a large part of the energy spectrum for some sources (van Kerkwijk , 2004). This makes the correct spectral fitting difficult. One needs to measure the interstellar absorption independently (for example, by simultaneous UV observation) to solve this problem. (2) The thermal component of the energy spectrum can vary in a timescale of months (Rutledge et al. , 2002). Moreover, in addition to the thermal emission, sometimes a non-thermal component is found (see, for example, Rutledge et al. (2002)). This nonthermal emission may be due to a residual accretion (van Kerkwijk , 2004). Detailed observations of many quiescent LMXBs with XMM-Newton, Chandra and future Xray instruments will be required to interpret the quiescent emission X-ray spectrum correctly, and hence to increase the reliability of the method. (3) Episodic higher accretion rates have been inferred during the quiescent emission (Kuulkers et al. , 2009). However, such high accretion is usually followed by a thermonuclear burst (Kuulkers et al. , 2009). Therefore if such a burst is detected, then the data immediately prior to it can be excluded from the neutron star parameter measurement analysis.

Finally we note that a neutron star heats up during accretion, and cools down when the system goes into the quiescence. The cooling curve of the neutron star can be useful to understand its properties (e.g., Wijnands et al. (2004)). Since repeated outbursts can be observed from a given transient system, the neutron star can heat up and cool down repeatedly, providing a useful tool to study the stellar core and crust.

\subsection{Mass Measurement: Binary Orbital Motion Method}

The mass $M$ of a neutron star affects the motion of the companion star via gravitation, and hence this mass may be constrained from the optical observations of the signatures of binary orbital motions. For example, the radial velocity (RV; i.e., the orbital velocity component along the line of sight) curve of the companion, based on, say, its absorption line spectra, can be useful to measure the binary orbital period $\left(P_{\text {orb }}\right)$ and the neutron star mass function:

$$
f(M)=\frac{P_{\mathrm{orb}} K_{\mathrm{comp}}^{3}}{2 \pi G}=\frac{M \sin ^{3} i}{(1+q)^{2}} .
$$

Here $K_{\text {comp }}$ is the amplitude of the companion star RV, $i$ is the observer's inclination angle, $q=M_{\text {comp }} / M$, and
$M_{\text {comp }}$ is the companion mass. Note that, since $q>0$ and $\sin i \leq 1, f(M)$ gives the lower limit of the neutron star mass. Furthermore, with the estimated $i$ and $q, M$ can be constrained from equation 14 . The inclination angle $i$ can be estimated from the observed light curves, and/or from X-ray dips/eclipses that may be detected from the LMXB (e.g., White and Swank (1982); Parmar et al. (1986)). For example, observations of both dips and total eclipses may imply $i \approx 75^{\circ}-80^{\circ}$ (Frank et al. , 1987). Dips and eclipses also provide the binary orbital period (e.g., White and Swank (1982); Parmar et al. (1986)). The mass ratio $q$ of equation 14 can be estimated by measuring the rotational (i.e., spin-induced) broadening of the spectral lines originated from the companion star. This is because the spin-related linear speed ( $v_{\text {comp }}$ ) of the companion surface can be expressed in terms of $q$ using the following formula (Wade and Horne, 1988):

$$
v_{\text {comp }} \sin i=0.462 K_{\text {comp }} q^{1 / 3}(1+q)^{2 / 3} \text {. }
$$

However, if an estimate of $q$ is not available, then one needs to measure the companion star mass function:

$$
f\left(M_{\mathrm{comp}}\right)=\frac{P_{\mathrm{orb}} K_{\mathrm{NS}}^{3}}{2 \pi G}=\frac{M_{\mathrm{comp}} \sin ^{3} i}{(1+[1 / q])^{2}}
$$

in order to constrain the neutron star mass. Here $K_{\mathrm{NS}}$ is the amplitude of the RV of the neutron-star.

Unfortunately, the optical emission of a neutron star LMXB is heavily dominated by the accretion disk radiation for most sources. This optical radiation originates from the reprocessing of the X-rays in the outer accretion disk. As a result, the above mentioned binary orbital motion method cannot be effectively used for the neutron star LMXBs, except for a few sources for which the companion star can be detected, and its relevant properties can be measured sufficiently well (e.g., Orosz and Kuulkers (1999); Jonker et al. (2005)). An example of such a system is $\mathrm{Cyg} \mathrm{X}-2$, because $\approx 70 \%$ of its optical emission originates from the companion star (Elebert et al. , 2009). The transient neutron star LMXBs in their quiescent state might therefore be ideal to apply the above mentioned method (see $\S 3.5$ ). But, at a distance of a few $\mathrm{kpc}$ the low-mass companion stars are usually too faint to observe, again making the binary orbital motion method ineffective.

In 2002, Steeghs and Casares (2002) discovered narrow high-excitation optical emission lines from the neutron star LMXB Sco X-1. The strongest lines were in the Bowen range $(4630 \AA-4660 \AA)$, that primarily consisted of a blend of N III and C III lines (see Figure 15). The $\mathrm{N}$ III lines originate from a UV fluorescence process, and the C III lines are due to photo-ionization and subsequent recombination (McClintock et al. , 1975). These lines are believed to originate from the irradiated surface of the companion star, mainly because of the following reasons: (1) the Doppler tomography of Bowen blend emission lines revealed a bright spot of emission (see Fig. 4 of 


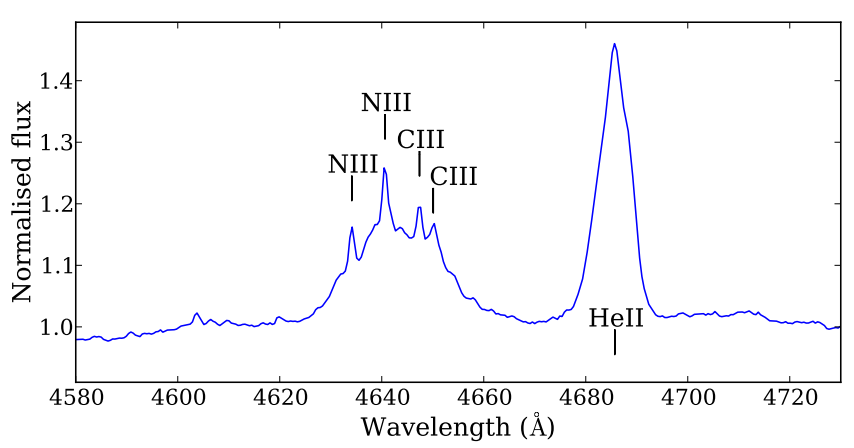

Figure 15: Average normalised optical spectrum of Sco X-1 in the rest frame of the companion star. The Bowen blend N III and C III emission lines from the irradiated surface of the companion star, and the He II emission line are shown ( $\$ 3.6$; figure courtesy: Danny Steeghs; Steeghs and Casares (2002)).

Steeghs and Casares (2002)); and (2) phase-resolved spectroscopy showed that these narrow lines moved roughly in anti-phase with the neutron-star/accretion-disk component (see Figure 16). As a result these lines led to the first estimate of $K_{\text {comp }}$ for a neutron star LMXB for which the optical emission primarily originates from the accretion disk. Therefore, the discovery of Steeghs and Casares (2002) and the subsequent applications of the above mentioned binary orbital motion method using the Bowen blend emission lines for several sources have established a new technique to constrain the neutron star masses (see Cornelisse et al. (2008) and references therein; see also Muñoz-Darias et al. (2009)).

This new technique, although is very promising, suffers from systematics mainly because of the unknown values of various source parameters. For example, $i$ and $q$ of equation 14 and $K_{\mathrm{NS}}$ of equation 16 are not known for many sources. Besides, $K_{\text {comp }}$ measured from the narrow Bowen blend emission lines is only a lower-limit to the true $K_{\text {comp }}$, because these lines arise on the irradiated side of the companion star which does not correspond to the center of mass of the companion. This shift of the measured $K_{\text {comp }}$ from the true $K_{\text {comp }}$ can be accounted for by the " $K$ correction", which depends on $i, q$ and the opening-angle of the accretion disk (Muñoz-Darias et al. (2005); see also de Jong et al. (1996)). Among these source parameters, the uncertainty on $i$ and the opening-angle of the accretion disk could be reduced by modeling the accretion disk, and $q$ could be obtained by clearly resolving the narrow lines to determine the rotational broadening. Another systematic uncertainty comes from the poorly measured systemic velocity, which is an important parameter to do Doppler tomography (e.g., Cornelisse et al. (2009)). Higher resolution data can be useful to reduce this uncertainty. Finally, the Bowen blend emission lines may also primarily originate from the gas-stream/accretion-disk impact region, rather than from the irradiated surface of the companion

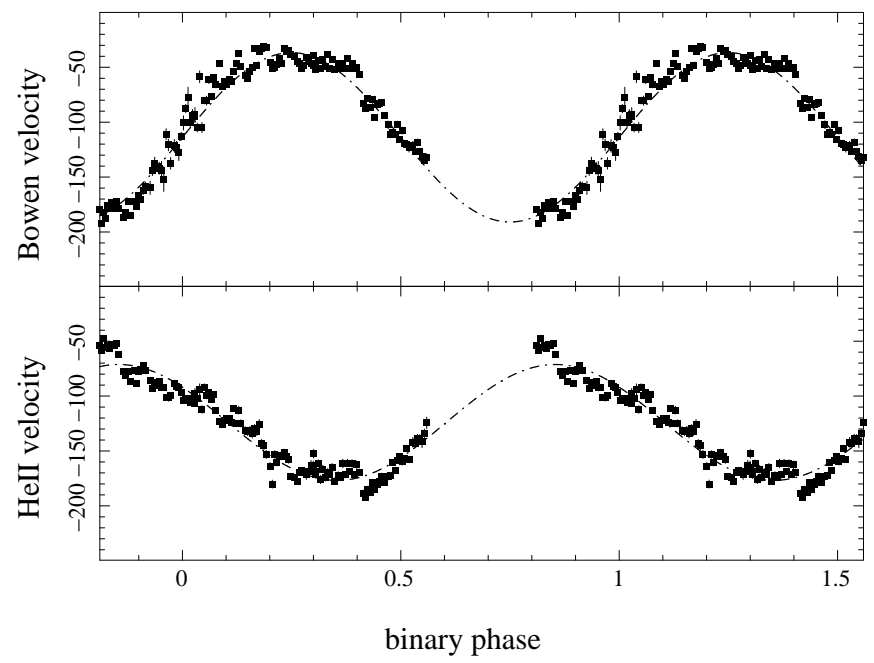

Figure 16: Upper panel: Radial velocity (RV; in $\mathrm{km} / \mathrm{s}$ ) curve of the narrow Bowen blend emission lines (see Figure 15) from Sco X1. Data are repeated over two cycles, and the best sinusoidal fit is plotted as a dash-dot line. Lower panel: Data and sinusoidal fit of the RV curve of the He II emission line (see Figure 15) from Sco X-1. Since the Bowen blend emission lines originate from the irradiated surface of the companion star, and the He II line comes from near the neutron star, the RV curve of the latter is naturally roughly in anti-phase with that of the former ( $\S 3.6$; figure courtesy: Danny Steeghs; Steeghs and Casares (2002)).

star, for some neutron star LMXBs (Elebert et al. , 2009).

\section{Why are Low-mass X-ray Binaries Useful?}

As mentioned in $\S 1$ (see also Figure 1), one needs to measure at least three independent parameters of the same neutron star in order to constrain the EoS models of the supranuclear core matter. Because of a number of systematics (mentioned throughout this review), it is possible only if several measurement methods can be used for a given neutron star. This may be achieved for neutron star LMXB systems, because many of them exhibit several of the following features: thermonuclear bursts without oscillations (suitable for continuum spectrum method), burst oscillations, PRE bursts, mHz QPOs, accretion-powered millisecond period pulsations, $\mathrm{kHz}$ QPOs, broad relativistic iron lines, quiescent emission, Bowen blend emission lines, etc. For example, SAX J1808.4-3658 has shown all these features except $\mathrm{mHz}$ QPOs, and 4U 1636-536 has exhibited all these features except accretion-powered pulsations and quiescent emission. Therefore, Bayesian method may be used to strongly constrain the neutron star parameter values, which do not change from one observation to another (see $\S 3.1 .8$ for a discussion). This is why LMXBs are useful systems to measure the neutron star parameters. In Table 1 we give examples of a few sources for which various methods mentioned in this review have been attempted, and in Figure 17 we demonstrate how two of these methods can be jointly used to tightly constrain the $M-R$ space. 


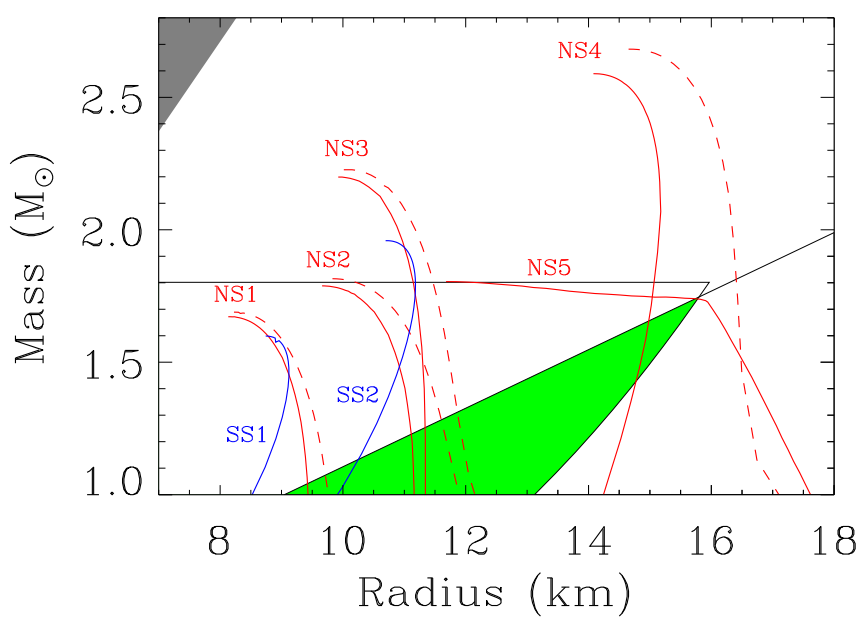

Figure 17: This figure shows the $M-R$ space of neutron stars with the curves corresponding to a few representative EoS models (same as Figure 1). Miller et al. (1999) mentioned that the highest $\mathrm{kHz}$ QPO frequency observed from the neutron star LMXB 4U 1636-536 was $1220 \mathrm{~Hz}$, which gives an allowed $M-R$ region for this source according to the equations 12 and 13 (compare this figure with Figure 13). In addition, Nath et al. (2002) found $G M / R c^{2}<0.163$ for $4 \mathrm{U} 1636-536$ by modeling the burst oscillations. The green patch shows the intesection of these two allowed regions, which should be the allowed $M-R$ space for $4 \mathrm{U} 1636-536$ using the kilohertz quasiperiodic oscillation method $(\S 3.3 .2)$ and the burst oscillation method (§ 3.1.6). Therefore, this figure demonstrates how various methods described throughout this review can be jointly used to tightly constrain the $M-R$ space. However, note that the particular allowed $M-R$ space shown here may not be very reliable, because the actual origin of $\mathrm{kHz}$ QPOs is not yet known, and the burst oscillation measurement of $M / R$ used here suffered from systematics.

There is another reason that makes neutron star LMXBs useful. In order to understand the nature of the supranuclear core matter extremely well, we will need to observationally trace out the stellar $M-R$ curve (see, for example, Özel and Psaltis (2009); see also Figure 1). Although, currently we cannot do it, with the technological advancement it may be doable in the future. But this will be possible only if neutron stars have a sufficiently large range of mass values. Since the neutron stars in LMXBs can accrete mass plausibly up to $0.7 M_{\odot}$ (e.g., van den Heuvel and Bitzaraki (1995)), they should occupy a larger range in the mass space than the isolated neutron stars, and the stars in double neutron star binaries. These latter neutron stars are formed in HMXBs, and hence a limited amount of matter can be accreted because of the short life of the massive companion. In fact, Thorsett and Chakrabarty (1999) found neutron star mass $M=1.35 \pm 0.04 M_{\odot}$ for a sample of double neutron star binaries. Besides, LMXBs may contain neutron stars massive enough to rule out the softer EoS models (see $\S 1$ ). Therefore, the LMXB systems may be intrinsically more useful than the isolated stars, and stars in double neutron star binary systems.

\section{Summary and Future Prospects}

In this review, we have described a few methods to constrain the neutron star parameters. We have chosen the methods which utilize various properties of thermonuclear bursts, accretion-powered millisecond pulsations, $\mathrm{kHz}$ QPOs, broad relativistic iron lines, quiescent emissions, and binary orbital motions. Some of these phenomena provide more than one method. For example, thermonuclear burst alone provides five methods. Throughout the review we have stressed that various systematic uncertainties hinder the accurate measurement of the neutron star parameters, and a joint analysis of several phenomena has a great potential to reduce these uncertainties. The large amount of archival neutron star LMXB data accumulated with the current and past X-ray space missions can be used for such a joint analysis, and this analysis can be further improved with the advanced statistical and other techniques (e.g., Noble and Nowak (2008)). These techniques may include pulsed phase spectroscopy, higher order timing analysis, and a higher capability spectral fitting recipe, which can, for example, handle the fitting of burst continuum spectra with a large number of realistic atmospheric models. However, such techniques should be complemented with theoretical investigations of the various phenomena. For example, astrophysicists and nuclear physicists should work more cohesively to understand the various properties of thermonuclear bursts. Numerical study of the inner accretion disk in order to jointly model the $\mathrm{kHz}$ QPOs and the broad relativistic iron lines will also be useful. Besides, processes of the channelled flow and the radiative transfer in AMPs should also be numerically investigated.

In addition to the archival data analysis complemented with advanced techniques and theoretical studies, the proposed X-ray space missions also hold a great promise. The Indian multiwavelength astronomy space mission Astrosat is planned to be launched in 2010, and will observe simultaneously in a wide energy range (optical to hard X-ray of $100 \mathrm{keV}$ ). Apart from this unprecedented capability, its LAXPC instrument should be able to detect and measure the high frequency timing features, such as $\mathrm{kHz}$ QPOs and accretion-powered pulsations, for the first time in hard Xrays (say, up to $\approx 50 \mathrm{keV}$ ). This will be useful, among other things, to discriminate among various $\mathrm{kHz}$ QPO modulation mechanism models. The Japanese X-ray satellite Astro- $H$ is scheduled to be launched in 2013. This mission will have an unprecedented imaging capability in the $0.3-80 \mathrm{keV}$ range, and will increase the reliability of the neutron star parameter measurement methods. The International X-ray Observatory (IXO), jointly proposed by NASA, ESA and JAXA, will have several highly sensitive instruments which will take the X-ray study of neutron star LMXBs to a higher level. The launch of this space mission is planned for 2021. The proposed "High Timing Resolution Spectrometer" (HTRS) of IXO will be able to observe sources with fluxes of $10^{6}$ counts per second in the $0.3-10 \mathrm{keV}$ band without performance degradation, 


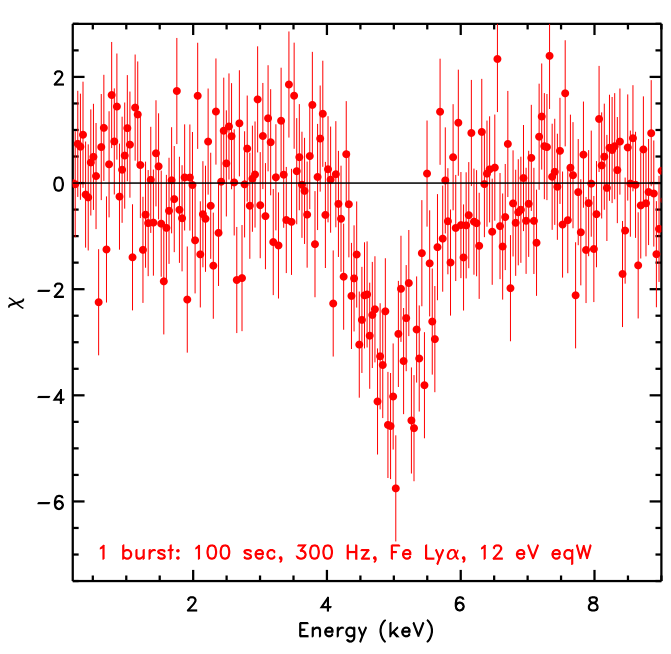

Figure 18: The residuals ( $\chi^{2}$ deviations) for a simulated iron Ly $\alpha$ absorption line from the neutron star surface during a thermonuclear $\mathrm{X}$-ray burst. The assumed values are the following: surface gravitational redshift $1+z=1.35$, line equivalent width $=12 \mathrm{eV}$, exposure $=100 \mathrm{~s}$, burst blackbody temperature $=1.7 \mathrm{keV}$ and X-ray flux $=$ $4 \times 10^{-8} \mathrm{ergs} \mathrm{s}^{-1} \mathrm{~cm}^{-2}$. The simulation was done for the "High Timing Resolution Spectrometer" (HTRS; previously proposed for the $X$-Ray Evolving Universe Spectrometer (XEUS), but now proposed for the International $X$-ray Observatory (IXO)). This figure shows that even a short observation of thermonuclear X-ray bursts with HTRS may lead to a significant detection of surface atomic spectral lines (§ 3.1.4; figure courtesy: Didier Barret; Barret et al. (2008)).

while providing good spectral resolution and excellent time resolution (e.g., Barret et al. (2008)). This instrument should, therefore, be able to reduce the systematics significantly, and measure the parameters of several neutron stars accurately. In Figures 18, 19 and 20, we show the simulated capabilities of HTRS. The Advanced X-ray Timing Array $(A X T A R)$, which is an X-ray observatory mission concept currently under study, will provide a better sensitivity than IXO for timing observations of accreting neutron stars (see Ray et al. (2009)). Finally, future large optical/IR telescopes, for example, the proposed $E x$ tremely Large Telescope (Spyromilio et al. , 2008), Thirty Meter Telescope (Nelson and Sanders , 2008), can be very useful for the binary orbital motion method, especially by detecting the companion stars of transient neutron star LMXBs in the quiescent state.

\section{Acknowledgments}

We thank Cole Miller for helpful suggestions which improved the manuscript, Remon Cornelisse for useful inputs regarding the binary orbital motion method, Arun Thampan for providing the code used to compute the structure of rapidly spinning neutron stars, and Didier Barret and Danny Steeghs for providing a few figures. We also thank the two reviewers for their very constructive comments.

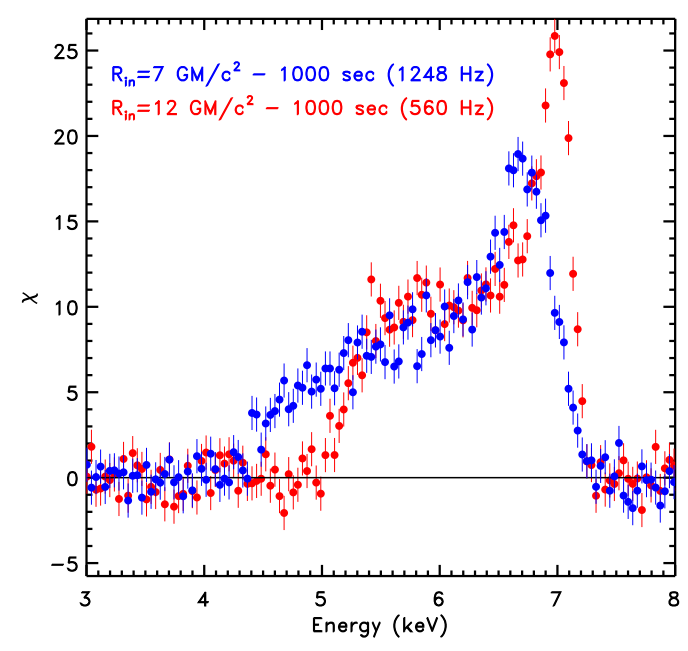

Figure 19: The residuals ( $\chi^{2}$ deviations) for simulated broad relativistic iron lines at two different inner accretion disk radii. The simulation was done for $1000 \mathrm{~s}$ of HTRS exposure. This figure shows that a short HTRS observation of relativistic lines may significantly reveal their detailed structures ( $\S 3.4$; figure courtesy: Didier Barret; Barret et al. (2008)).

This work was supported in part by US NSF grant AST 0708424.

\section{References}

Abramowicz, M. A., Bulik, T., Bursa, M., \& Kluźniak, W., Evidence for a 2:3 resonance in Sco X-1 kHz QPOs, A\&A, 404, L21-L24, 2003.

Abramowicz, M.A., \& Kluźniak, W., A precise determination of black hole spin in GRO J1655-40, A\&A, 374, L19-L20, 2001.

Akmal, A., Pandharipande, V.R., \& Ravenhall, D.G., Equation of state of nucleon matter and neutron star structure, Physical Review C, 58, 1804-1828, 1998.

Altamirano, D., Linares, M., Patruno, A., et al., Type I X-ray bursts, burst oscillations and $\mathrm{kHz}$ quasi-periodic oscillations in the neutron star system IGR J17191-2821, MNRAS, in press, 2009.

Altamirano, D., Strohmayer, T.E., Heinke, C.O., et al., Discovery of a $205 \mathrm{~Hz}$ X-ray pulsar in the globular cluster NGC 6440, ATEL 2182, 2009.

Altamirano, D., van der Klis, M., Wijnands, R., \& Cumming, A., Millihertz Oscillation Frequency Drift Predicts the Occurrence of Type I X-Ray Bursts, ApJ, 673, L35-L38, 2008.

Asai, K., Dotani, T., Nagase, F., \& Mitsuda, K., Iron K Emission Lines in the Energy Spectra of Low-Mass X-Ray Binaries Observed with ASCA, ApJS, 131, 571-591, 2000.

Ayasli, S., \& Joss, P.C., Thermonuclear processes on accreting neutron stars - A systematic study, ApJ, 256, 637-665, 1982.

Bagchi, M., Ray, S., Dey, M., \& Dey, J., Evidence for strange stars from joint observation of harmonic absorption bands and of redshift, MNRAS, 368, 971-975, 2006.

Baldo, M., Bombaci, I., \& Burgio, G.F., Microscopic nuclear equation of state with three-body forces and neutron star structure, A\&A, 328, 274-282, 1997.

Balman, S., Frequency Resolved Spectroscopy of EXO 0748-676 in Persistent Emission, ATel, 2097, 2009.

Barnes, A. D., Casares, J., Cornelisse, R., Charles, P. A., Steeghs, D., Hynes, R. I., \& O'Brien, K., Kinematical studies of the lowmass X-ray binary GRMus (XB1254-690), MNRAS, 380, 11821190, 2007. 


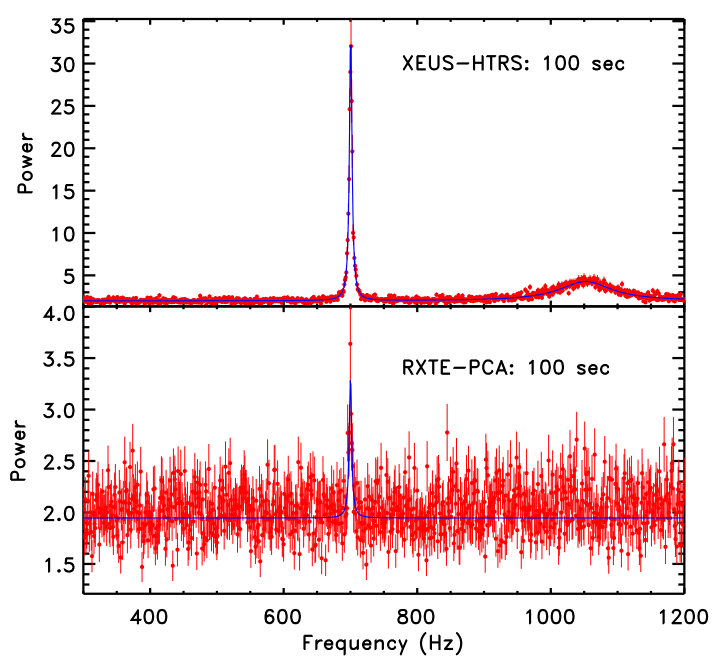

Figure 20: A comparison of simulated power spectra observed with two instruments (one current and one future). The lower panel shows that the lower $\mathrm{kHz}$ QPO is barely detected and the upper $\mathrm{kHz} \mathrm{QPO}$ is not detected from the power spectrum created from an intensity profile of $100 \mathrm{~s}$ duration observed with the $R X T E$ PCA. The upper panel shows that, if the same observation were made with the HTRS, both the $\mathrm{kHz}$ QPOs would be very significantly detected ( $\S 3.3$; figure courtesy: Didier Barret; Barret et al. (2008)).

Barret, D., Belloni, T., Bhattacharyya, S., et al., Science with the XEUS high time resolution spectrometer, SPIE, 7011, 1-10, 2008. Barret, D., Olive, J.-F., \& Miller, M.C., The coherence of kilohertz quasi-periodic oscillations in the X-rays from accreting neutron stars, MNRAS, 370, 1140-1146, 2006.

Basinska, E.M., Lewin, W.H.G., Sztajno, M., Cominsky, L.R., \& Marshall, F.J., X-ray observations of the burst source MXB 172834, ApJ, 281, 337-353, 1984.

Belian, R.D., Conner, J.P., \& Evans, W. D., X-ray bursts and neutron-star thermonuclear flashes, ApJ, 206, L135-L138, 1976.

Bhattacharya, D., \& van den Heuvel, E.P.J., Formation and evolution of binary and millisecond radio pulsars, Physics Reports, 203, 1-124, 1991.

Bhattacharyya, S., Limits to the Mass and the Radius of the Compact Star in SAX J1808.4-3658 and Their Implications, ApJ, 554, L185-L188, 2001.

Bhattacharyya, S., Timing properties of XB 1254-690, MNRAS, 377, 198-202, 2007.

Bhattacharyya, S., Simultaneous observations of a pair of kilohertz QPOs and a plausible $1860 \mathrm{~Hz}$ QPO from an accreting neutron star system, RAA, in press, 2009.

Bhattacharyya, S., Bhattacharya, D., \& Thampan, A. V., General relativistic spectra of accretion discs around rapidly rotating neutron stars: effect of light bending, MNRAS, 325, 989-994, 2001.

Bhattacharyya, S., Miller, M.C., \& Galloway, D.K., Systematic variation in the apparent burning area of thermonuclear bursts and its implication for neutron star radius measurement, MNRAS, in press, 2009.

Bhattacharyya, S., Miller, M.C., \& Lamb, F.K., The Shapes of Atomic Lines from the Surfaces of Weakly Magnetic Rotating Neutron Stars and Their Implications, ApJ, 644, 1085-1089, 2006.

Bhattacharyya, S., Misra, R., \& Thampan, A. V., General Relativistic Spectra of Accretion Disks around Rotating Neutron Stars, ApJ, 550, 841-845, 2001.

Bhattacharyya, S., \& Strohmayer, T.E., Evidence for Harmonic Content and Frequency Evolution of Oscillations during the Rising Phase of X-Ray Bursts from 4U 1636-536, ApJ, 634, L157-L160, 2005.
Bhattacharyya, S., \& Strohmayer, T.E., A Non-PRE Double-peaked Burst from 4U 1636-536: Evidence of Burning Front Propagation, ApJ, 636, L121-L124, 2006.

Bhattacharyya, S., \& Strohmayer, T.E., Signature of Temporary Burning Front Stalling from a Non-Photospheric Radius Expansion Double-peaked Burst, ApJ, 641, L53-L56, 2006.

Bhattacharyya, S., \& Strohmayer, T.E., Spreading of Thermonuclear Flames on the Neutron Star in SAX J1808.4-3658: An Observational Tool, ApJ, 642, L161-L164, 2006.

Bhattacharyya, S., \& Strohmayer, T.E., An Unusual Precursor Burst with Oscillations from SAX J1808.4-3658, ApJ, 656, 414-419, 2007.

Bhattacharyya, S., \& Strohmayer, T.E., Evidence of a Broad Relativistic Iron Line from the Neutron Star Low-Mass X-Ray Binary Serpens X-1, ApJ, 664, L103-L106, 2007.

Bhattacharyya, S., \& Strohmayer, T.E., Thermonuclear Flame Spreading on Rapidly Spinning Neutron Stars: Indications of the Coriolis Force? ApJ, 666, L85-L88, 2007.

Bhattacharyya, S., Strohmayer, T.E., Markwardt, C.B., \& Swank, J.H., The Discovery of a Neutron Star with a Spin Frequency of $530 \mathrm{~Hz}$ in A1744-361, ApJ, 639, L31-L34, 2006.

Bhattacharyya, S., Strohmayer, T. E., Swank, J. H., \& Markwardt, C. B., RXTE Observations of 1A 1744-361: Correlated Spectral and Timing Behavior, ApJ, 652, 603-609, 2006.

Bhattacharyya, S., Strohmayer, T.E., Miller, M.C., \& Markwardt, C.B., Constraints on Neutron Star Parameters from Burst Oscillation Light Curves of the Accreting Millisecond Pulsar XTE J1814-338, ApJ, 619, 483-491, 2005.

Bhattacharyya, S., Thampan, A.V., \& Bombaci, I., Temperature profiles of accretion discs around rapidly rotating strange stars in general relativity: A comparison with neutron stars, A\&A, 372, 925-934, 2001.

Bhattacharyya, S., Thampan, A.V., Misra, R., \& Datta, B., Temperature Profiles of Accretion Disks around Rapidly Rotating Neutron Stars in General Relativity and the Implications for Cygnus X-2, ApJ, 542, 473-483, 2000.

Bildsten, L., Rings of Fire: Nuclear Burning as the Origin of SubHertz Noise and Weak X-Ray Bursts in Accreting i Neutron Stars, ApJ, 418, L21-L24, 1993.

Bildsten, L., Theory and observations of Type I X-Ray bursts from neutron stars, AIP Conference Proceedings, 522, 359-369, 2000.

Bildsten, L., \& Chakrabarty, D., A Brown Dwarf Companion for the Accreting Millisecond Pulsar SAX J1808.4-3658, ApJ, 557, 292-296, 2001.

Blaschke, D., Klaehn, T., \& Weber, F., Constraints on the HighDensity Nuclear Equation of State from Neutron Star Observables, Contribution to Proceedings of the 3rd International Workshop on Astronomy and Relativistic Astrophysics (IWARA), 2007.

Bombaci, I., Observational evidence for strange matter in compact objects from the x-ray burster $4 \mathrm{U}$ 1820-30, Physical Review C, 55, 1587-1590, 1997.

Bombaci, I., Thampan, A.V., \& Datta, B., Rapidly Rotating Strange Stars for a New Equation of State of Strange Quark Matter, ApJ, 541, L71-L74, 2000.

Braje, T.M., Romani, R.W., \& Rauch, K.P., Light Curves of Rapidly Rotating Neutron Stars, ApJ, 531, 447-452, 2000.

Brenneman, L.W., \& Reynolds, C.S., Constraining Black Hole Spin via X-Ray Spectroscopy, ApJ, 652, 1028-1043, 2006.

Brown, E.F., \& Bildsten, L., The Ocean and Crust of a Rapidly Accreting Neutron Star: Implications for Magnetic Field Evolution and Thermonuclear Flashes, ApJ, 496, 915-933, 1998,

Burderi, L., \& King, A.R., A Firm Upper Limit to the Radius of the Neutron Star in SAX J1808.4-3658, ApJ, 505, L135-L137, 1998.

Cackett, E.M., Altamirano, D., Patruno, A., et al., Broad Relativistic Iron Emission Line Observed in SAX J1808.4-3658, ApJ, 694, L21-L25, 2009.

Cackett, E.M., Miller, J.M., Ballantyne, D.R., et al., Relativistic Lines and Reflection from the Inner Accretion Disks Around Neutron Stars, submitted, 2009 (arXiv:0908.1098).

Cackett, E.M., Miller, J.M., Bhattacharyya, S., et al., Relativistic Iron Emission Lines in Neutron Star Low-Mass X-Ray Binaries as 
Probes of Neutron Star Radii, ApJ, 674, 415-420, 2008.

Cackett, E.M., Wijnands, R., Miller, J.M., Brown, E.F., \& Degenaar, N., Cooling of the Crust in the Neutron Star Low-Mass X-Ray Binary MXB 1659-29, ApJ, 687, L87-L90, 2008.

Cadeau, C., Morsink, S.M., Leahy, D., \& Campbell, S.S., Light Curves for Rapidly Rotating Neutron Stars, ApJ, 654, 458-469, 2007.

Casella, P., Altamirano, D., Patruno, A., Wijnands, R., \& van der Klis, M., Discovery of Coherent Millisecond X-Ray Pulsations in Aquila X-1, ApJ, 674, L41-L44, 2008.

Chakrabarty, D., \& Morgan, E.H., The two-hour orbit of a binary millisecond X-ray pulsar, Nature, 394, 346-348, 1998.

Chakrabarty, D., Morgan, E.H., Muno, M.P., Galloway, D.K., Wijnands, R., van der Klis, M., \& Markwardt, C.B., Nuclear-powered millisecond pulsars and the maximum spin frequency of neutron stars, Nature, 424, 42-44, 2003.

Chang, P., Bildsten, L., \& Wasserman, I., Formation of Resonant Atomic Lines during Thermonuclear Flashes on Neutron Stars, ApJ, 629, 998-1007, 2005.

Chang, P., Morsink, S., Bildsten, L., \& Wasserman, I., Rotational Broadening of Atomic Spectral Features from Neutron Stars, ApJ, 636, L117-L120, 2006.

Chevalier, C., \& Ilovaisky, S.A., EXOSAT observations of a long X-ray burst from XB 1905 + 000, A\&A, 228, 115-124, 1990.

Choudhuri, A. R., \& Konar, S., Diamagnetic screening of the magnetic field in accreting neutron stars, MNRAS, 332, 933-944, 2002

Cook, G.B., Shapiro, S.L., \& Teukolsky, S.A., Rapidly rotating neutron stars in general relativity: Realistic equations of state, ApJ, 424, 823-845, 1994

Cooper, R.L., Mukhopadhyay, B., Steeghs, D., \& Narayan, R., On the Production and Survival of Carbon Fuel for Superbursts on Accreting Neutron Stars: Implications for Mass Donor Evolution, ApJ, 642, 443-454, 2006.

Cooper, R.L., \& Narayan, On the Physics of Type I X-Ray Bursts on Accreting Neutron Stars at High Accretion Rates, ApJ, 648, L123-L126, 2006.

Cooper, R.L., \& Narayan, The Latitude of Type I X-Ray Burst Ignition on Rapidly Rotating Neutron Stars, ApJ, 657, L29-L32, 2007.

Cooper, R.L., \& Narayan, Hydrogen-triggered Type I X-Ray Bursts in a Two-Zone Model, ApJ, 661, 468-476, 2007.

Cooper, R.L., Steiner, A.W., \& Brown, E.F., Possible Resonances in the ${ }^{12} \mathrm{C}+{ }^{12} \mathrm{C}$ Fusion Rate and Superburst Ignition, ApJ, in press, 2009.

Cornelisse, R., Casares, J., Muñoz-Darias, T., Steeghs, D., Charles, P., Hynes, R., O'Brien, K., \& Barnes, A., An Overview of the Bowen Survey: Detecting Donor Star Signatures in Low Mass Xray Binaries, AIP Conference Proceedings, 1010, 148-152, 2008.

Cornelisse, R., D'Avanzo, P., Muñoz-Darias, T., et al., Phaseresolved spectroscopy of the accreting millisecond X-ray pulsar SAX J1808.4-3658 during the 2008 outburst, A\&A, 495, L1-L4 2009.

Cornelisse, R., Heise, J., Kuulkers, E., Verbunt, F., \& in't Zand, J.J.M., The longest thermonuclear X-ray burst ever observed? A BeppoSAX Wide Field Camera observation of $4 \mathrm{U}$ 1735-44, A\&A, 357, L21-L24, 2000.

Cottam, J., Paerels, F., \& Méndez, M., Gravitationally redshifted absorption lines in the X-ray burst spectra of a neutron star Nature, 420, 51-54, 2002.

Cottam, J., Paerels, F., \& Méndez, M., Boirin, L., Lewin, W.H.G., Kuulkers, E., \& Miller, J.M., The Burst Spectra of EXO 0748676 during a Long 2003 XMM-Newton Observation, ApJ, 672, 504-509, 2008

Cui, W., Morgan, E.H., \& Titarchuk, L.G., Soft Phase Lags in the Millisecond X-Ray Pulsar SAX J1808.4-3658, ApJ, 504, L27-L30, 1998.

Cumming, A., Models of Type I X-Ray Bursts from 4U 1820-30, ApJ, 595, 1077-1085, 2003.

Cumming, A., Latitudinal Shear Instabilities during Type I X-Ray Bursts, ApJ, 630, 441-453, 2005.

Cumming, A., \& Bildsten, L., Carbon Flashes in the Heavy-Element
Ocean on Accreting Neutron Stars, ApJ, 559, L127-L130, 2001.

D’Aí, A., Iaria, R., Di Salvo, T., Matt, G., \& Robba, N.R., Disk Reflection Signatures in the Spectrum of the Bright Z-Source GX 340+0, ApJ, 693, L1-L5, 2009

Damen, E., Magnier, E., Lewin, W.H.G., Tan, J., Penninx, W., \& van Paradijs, J., X-ray bursts with photospheric radius expansion and the gravitational redshift of neutron stars, A\&A, 237, 103-109, 1990.

Datta, B., Thampan, A.V., \& Bombaci, I., Equilibrium sequences of rotating neutron stars for new realistic Equations of State, A\&A, 334, 943-952, 1998.

de Jong, J. A., van Paradijs, J., \& Augusteijn, T., Reprocessing of $\mathrm{X}$ rays in low-mass X-ray binaries, A\&A, 314, 484-490, 1996.

di Salvo, T., D'Aí, A., \& Iaria, R., et al., A relativistically smeared spectrum in the neutron star X-ray binary $4 \mathrm{U}$ 1705-44: looking at the inner accretion disc with X-ray spectroscopy, MNRAS, in press, 2009.

Díaz Trigo, M., Parmar, A.N., Boirin, L., Méndez, M., \& Kaastra, J.S., Spectral changes during dipping in low-mass X-ray binaries due to highly-ionized absorbers, A\&A, 445, 179-195, 2006.

Ebisuzaki, T., X-ray spectra and atmospheric structures of bursting neutron stars, PASJ, 39, 287-308, 1987.

Ebisuzaki, T., \& Nakamura, N., The difference in hydrogen abundance between two classes of type I X-ray bursts, ApJ, 328, 251255, 1988.

Elebert, P., Callanan, P. J., Torres, M. A. P., \& Garcia, M. R. Optical spectroscopy and Doppler tomography of Cygnus X-2, MNRAS, 395, 2029-2038, 2009.

Fabian, A.C., Iwasawa, K., Reynolds, C.S., \& Young, A.J., Broad Iron Lines in Active Galactic Nuclei, PASP, 112, 1145-1161, 2000.

Farhi, E., \& Jaffe, R.L., Strange matter, Physical Review D, 30, 2379-2390, 1984

Fisker, J.L., Görres, J., Wiescher, M., \& Davids, B., The Importance of ${ }^{15} \mathrm{O}(\alpha, \gamma){ }^{19} \mathrm{Ne}$ to X-Ray Bursts and Superbursts, ApJ, 650, 332-337, 2006.

Fisker, J.L., Schatz, H., \& Thielemann, F.-K., Explosive Hydrogen Burning during Type I X-Ray Bursts, ApJSS, 174, 261-276, 2008.

Fowler, W.A., \& Hoyle, F., Nucleosynthesis in massive stars and supernovae, University of Chicago Press (Chicago, 1965).

Frank, J., King, A. R., \& Lasota, J.-P., The light curves of low-mass X-ray binaries, A\&A, 178, 137-142, 1987.

Fryxell, B.A., \& Woosley, S.E., Finite propagation time in multidimensional thermonuclear runaways, ApJ, 261, 332-336, 1982.

Fujimoto, M.Y., \& Taam, R.E., The mass and radius of a neutron star in X-ray burster MXB 1636 - 536, ApJ, 305, 246-250, 1986.

Galloway, D.K., Chakrabarty, D., \& Lin, J.R., A 552Hz X-ray burst oscillation in the accreting neutron star EXO 0748-676, Atel, 2094, 2009 .

Galloway, D.K., Chakrabarty, D., Muno, M.P., \& Savov, P., Discovery of a 270 Hertz X-Ray Burst Oscillation in the X-Ray Dipper 4U 1916-053, ApJ, 549, L85-L88, 2001.

Galloway, D.K., \& Cumming, A., Helium-rich Thermonuclear Bursts and the Distance to the Accretion-powered Millisecond Pulsar SAX J1808.4-3658, ApJ, 652, 559-568, 2006.

Galloway, D.K., Muno, M.P., Hartman, J.M., Psaltis, D., \& Chakrabarty, D., Thermonuclear (Type I) X-Ray Bursts Observed by the Rossi X-Ray Timing Explorer, ApJSS, 179, 360-422, 2008.

Galloway, D.K., Özel, F., \& Psaltis, D., Biases for neutron star mass, radius and distance measurements from Eddington-limited X-ray bursts, MNRAS, 387, 268-272, 2008.

Galloway, D.K., Psaltis, D., Chakrabarty, D., \& Muno, M.P., Eddington-limited X-Ray Bursts as Distance Indicators. I. Systematic Trends and Spherical Symmetry in Bursts from $4 \mathrm{U} 1728$ 34, ApJ, 590, 999-1007, 2003.

Galloway, D.K., Psaltis, D., Muno, M.P., \& Chakrabarty, D. Eddington-limited X-Ray Bursts as Distance Indicators. II. Possible Compositional Effects in Bursts from 4U 1636-536, ApJ, 639, 1033-1038, 2006

Gendre, B., Barret, D., \& Webb, N., Discovery of a quiescent neutron star binary in the globular cluster M 13, A\&A, 403, L11-L14, 2003.

Gilfanov, M., Revnivtsev, M., Sunyaev, R., \& Churazov, E., The 
millisecond X-ray pulsar/burster SAX J1808.4-3658: the outburst light curve and the power law spectrum, A\&A, 338, L83-L86, 1998.

Goldman, I., General relativistic effects and the radius and mass of X-ray bursters, A\&A, 78, L15-L16, 1979.

Gottwald, M., Haberl, F., Parmar, A.N., \& White, N.E., The properties of bursts with short recurrence times from the transient X-ray source EXO 0748-676, ApJ, 323, 575-581, 1987.

Grindlay, J.E., et al., Discovery of intense X-ray bursts from the globular cluster NGC 6624, ApJ, 205, L127-L130, 1976.

Guillot, S., Rutledge, R.E., Bildsten, L., Brown, E.F., Pavlov, G.G., \& Zavlin, V.E., X-ray spectral identification of three candidate quiescent low-mass X-ray binaries in the globular cluster NGC 6304, MNRAS, 392, 665-681, 2009.

Haberl, F., \& Titarchuk, L., On the distance and mass-radius relation of neutron stars in X-ray burst sources, A\&A, 299, 414-420, 1995.

Hanawa, T., \& Fujimoto, M.Y., Thermal response of neutron stars to shell flashes PASJ, 36, 199-214, 1984.

Hanawa, T., Sugimoto, D., \& Hashimoto, M., Nucleosynthesis in explosive hydrogen burning and its implications in ten-minute interval of X-ray bursts, PASJ, 35, 491-506, 1983.

Hansen, C. J., \& van Horn, H. M., Steady-state nuclear fusion in accreting neutron-star envelopes, ApJ, 195, 735-741, 1975.

Hartman, J.M., Watts, A.L., \& Chakrabarty, D., The Luminosity and Energy Dependence of Pulse Phase Lags in the Accretionpowered Millisecond Pulsar SAX J1808.4-3658, ApJ, 697, 21022107, 2009.

Heger, A., Cumming, A., Galloway, D.K., Woosley, S.E., Models of Type I X-Ray Bursts from GS 1826-24: A Probe of rp-Process Hydrogen Burning, ApJ, 671, L141-L144, 2007.

Heger, A., Cumming, A., \& Woosley, S.E., Millihertz Quasi-periodic Oscillations from Marginally Stable Nuclear Burning on an Accreting Neutron Star, ApJ, 665, 1311-1320, 2007.

Heinke, C.O., Jonker, P.G., Wijnands, R., Deloye, C.J., \& Taam, R.E., Further Constraints on Thermal Quiescent X-Ray Emission from SAX J1808.4-3658, ApJ, 691, 1035-1041, 2009.

Heng, K., \& Spitkovsky, A., Magnetohydrodynamic Shallow Water Waves: Linear Analysis, arXiv:0908.0946, 2009

Heyl, J.S., R-modes on rapidly rotating, relativistic stars - II. Blackbody emission, MNRAS, 361, 504-510, 2005.

Hoffman, J.A., Lewin, W.H.G., \& Doty, J., Further observations of the burst source MXB 1728-34, MNRAS, 179, 57-64, 1977.

Hoffman, J.A., Lewin, W.H.G., \& Doty, J., Observations of the X-ray burst source MXB 1636-53, ApJ, 217, L23-L28, 1977.

Iaria, R., D'Aí, A., Di Salvo, T., et al., A ionized reflecting skin above the accretion disk of GX 349+2, A\&A, in press, 2009.

Ibragimov, A., \& Poutanen, J., Accreting millisecond pulsar SAX J1808.4-3658 during its 2002 outburst: evidence for a receding disc, MNRAS, 400, 492-508, 2009

Inogamov, N.A., \& Sunyaev, R.A., Spread of matter over a neutronstar surface during disk accretion, AstL, 25, 269-293, 1999.

in't Zand, J.J.M., Bassa, C.G., Jonker, P.G., Keek, L., Verbunt, F., Mndez, M., \& Markwardt, C.B., An X-ray and optical study of the ultracompact X-ray binary A 1246-58, A\&A, 485, 183-194, 2008.

in't Zand, J.J.M., Cornelisse, R., \& Cumming, A., Superbursts at near-Eddington mass accretion rates, A\&A, 426, 257-265, 2004.

in't Zand, J.J.M., Cumming, A., van der Sluys, M.V., Verbunt, F., \& Pols, O.R., On the possibility of a helium white dwarf donor in the presumed ultracompact binary 2S 0918-549, A\&A, 441, 675-684, 2005 .

in't Zand, J.J.M., Verbunt, F., \& Kuulkers, E., Discovery of the neutron star nature of SLX 1737-282, A\&A, 389, L43-L46, 2002.

Jahan Miri, M., \& Bhattacharya, D., Evolution of the Magnetic Fields of Neutron Stars in Low-mass Binary Systems, JApAS, 16, 231-233, 1995.

Jimenez-Garate, M.A., Schulz, N.S., \& Marshall, H.L., Discrete XRay Signatures of a Photoionized Plasma above the Accretion Disk of the Neutron Star EXO 0748-676, ApJ, 590, 432-444, 2003.

Jonker, P.G., Steeghs, D., Nelemans, G., van der Klis, M., The radial velocity of the companion star in the low-mass X-ray binary $2 \mathrm{~S}$ 0921-630: limits on the mass of the compact object, MNRAS, 356,
621-626, 2005.

Jonker, P.G., van der Klis, M., Homan, J., et al., Discovery of a Kilohertz Quasi-periodic Oscillation in 2S 0918-549, ApJ, 553, 335-340, 2001.

Joss, P.C., X-ray bursts and neutron-star thermonuclear flashes, Nature, 270, 310-314, 1977.

Kaminker, A.D., Pavlov, G.G., Shibanov, I.A., Kurt, V.G., \& Shafer, E.I., ASTRON observations of the rapid burster MXB 1730-335 and constraints on burster parameters from spectra of trailing bursts, Ap\&SS, 173, 171-189, 1990.

Kaptein, R.G., in't Zand, J.J.M., Kuulkers, E., Verbunt, F., Heise, J., \& Cornelisse, R., Discovery of 1RXS J171824.2-402934 as an X-ray burster, A\&A, 358, L71-L74, 2000.

Keek, L., in't Zand, J.J.M., Kuulkers, E., Cumming, A., Brown, E.F., \& Suzuki, M., First superburst from a classical low-mass X-ray binary transient, A\&A, 479, 177-188, 2008.

Keek, L., Langer, N., \& in 't Zand, J.J.M., The effect of rotation on the stability of nuclear burning in accreting neutron stars, A\&A, in press, 2009.

King, A. R., Disc Instabilities in Soft X-ray Transients, Proceedings of the Jan van Paradijs Memorial Symposium, 2001.

Klein, R.I., Arons, J., Jernigan, G., \& Hsu, J.J.-L., Photon Bubble Oscillations in Accretion-powered Pulsars, ApJ, 457, L85-L89, 1996.

Klein, R.I., Jernigan, J.G., Arons, J., Morgan, E.H., \& Zhang, W., GRO J1744-28 and Scorpius X-1: First Evidence for Photon Bubble Oscillations and Turbulence, ApJ, 469, L119-L123, 1996.

Kluźniak, W., \& Abramowicz, M.A., Strong-Field Gravity and Orbital Resonance in Black Holes and Neutron Stars - $\mathrm{kHz}$ QuasiPeriodic Oscillations (QPO), Acta Physica Polonica B, 32, 36053612, 2001

Koike, O., Hashimoto, M., Arai, K., Wanajo, S., Rapid proton capture on accreting neutron stars - effects of uncertainty in the nuclear process, A\&A, 342, 464-473, 1999.

Konar, S., Bhattacharya, D., \& Urpin, V., Evolution of the Magnetic Field of an Accreting Neutron Star, JApAS, 16, 249-250, 1995.

Konar, S., \& Choudhuri, A. R., Diamagnetic screening of the magnetic field in accreting neutron stars - II. The effect of polar cap widening, MNRAS, 348, 661-668, 2004.

Kong, A.K.H., Miller, J.M., Méndez, M., et al., Nondetection of Gravitationally Redshifted Absorption Lines in the X-Ray Burst Spectra of GS 1826-24, ApJ, 670, L17-L20, 2007.

Kuulkers, E., The observers' view of (very) long X-ray bursts: they are super!, NuPhS, 132, 466-475, 2004.

Kuulkers, E., Probable superbursts in $4 \mathrm{U} 0614+091$ and $4 \mathrm{U} 1608-$ 522, ATel, 483, 1-1, 2005.

Kuulkers, E., den Hartog, P.R., in't Zand, J.J.M., Verbunt, F.W.M., Harris, W.E., \& Cocchi, M., Photospheric radius expansion X-ray bursts as standard candles, A\&A, 399, 663-680, 2003.

Kuulkers, E., in't Zand, J.J.M., \& Lasota, J.-P., Restless quiescence: thermonuclear flashes between transient X-ray outbursts, A\&A, 503, 889-897, 2009.

Kuulkers, E., \& van der Klis, M., The first radius-expansion X-ray burst from GX 3+1, A\&A, 356, L45-L48, 2000.

Leahy, D.A., Morsink, S.M., \& Cadeau, C., Limits on Mass and Radius for the Millisecond-Period X-Ray Pulsar SAX J1808.43658, ApJ, 672, 1119-1126, 2008.

Leahy, D.A., Morsink, S.M., Chung, Y.-Y., \& Chou, Y., Constraints on the Properties of the Neutron Star XTE J1814-338 from PulseShape Models, ApJ, 691, 1235-1242, 2009.

Lamb, F.K., \& Boutloukos, S., Accreting Neutron Stars in Low-Mass X-Ray Binary Systems, in Short-Period Binary Stars: Observations, Analyses, and Results, Eds. E.F. Milone, D.A. Leahy and D.W. Hobill, (Springer: Berlin, Germany), 352, 87, 2008.

Lamb, F.K., Boutloukos, S., Van Wassenhove, S., Chamberlain, R.T., Lo, K.H., Clare, A., Yu, W., \& Miller, M.C., A model for the waveform behavior of accreting millisecond pulsars: Nearly aligned magnetic fields and wandering emission regions, arXiv:0808.4159, 2008

Lamb, F.K., Boutloukos, S., Van Wassenhove, S., Chamberlain, R.T., Lo, K.H., \& Miller, M.C., Origin of intermittent accretion- 
powered oscillations in neutron stars with millisecond spin periods, arXiv:0809.4016, 2008.

Lamb, D.Q., \& Lamb, F.K., Nuclear burning in accreting neutron stars and X-ray bursts, ApJ, 220, 291-302, 1978.

Lamb, F.K., \& Miller, M.C., Sonic-Point and Spin-Resonance Model of the Kilohertz QPO Pairs, submitted (astro-ph/0308179), 2003.

Lattimer, J.M., \& Prakash, M., Neutron star observations: Prognosis for equation of state constraints, Physics Reports, 442, 109-165, 2007.

Lee, U., \& Strohmayer, T.E., Light curves of oscillating neutron stars, MNRAS, 361, 659-672, 2005

Lee, W.H., Abramowicz, M.A., \& Kluźniak, W., Resonance in Forced Oscillations of an Accretion Disk and Kilohertz Quasi-periodic Oscillations, ApJ, 603, L93-L96, 2004.

Lewin, W.H.G., Vacca, W.D., \& Basinska, E.M., Precursors to Xray bursts - The result of expansion and subsequent contraction of the neutron star's photosphere, ApJ, 277, L57-L60, 1984.

Lewin, W.H.G., van Paradijs, J., \& Taam, R.E., X-Ray Bursts, Space Sci. Rev., 62, 223-389, 1993.

Lewin, W.H.G., van Paradijs, J., \& Taam, R.E., X-Ray Bursts, in X-ray Bimaries, Eds. W.H.G. Lewin, J. van Paradijs and E.P.J. van den Heuvel, (Cambridge University Press), 175-232, 1995.

Li, X.-D., Bombaci, I., Dey, M., Dey, J., \& van den Heuvel, E.P.J., Is SAX J1808.4-3658 a Strange Star?, Physical Review Letters, 83, 3776-3779, 1999.

Lin, D.J., Bayliss, A., \& Taam, R.E., Low Mach Number Modeling of Type I X-Ray Burst Deflagrations, ApJ, 653, 545-557, 2006.

Liu, Q.Z., van Paradijs, J., \& van den Heuvel, E.P.J., A catalogue of low-mass X-ray binaries in the Galaxy, LMC, and SMC (Fourth edition), A\&A, 469, 807-810, 2007.

London, R.A., Howard, W.M., \& Taam, R.E., The spectra of X-ray bursting neutron stars, ApJ, 287, L27-L30, 1984.

London, R.A., Taam, R.E., \& Howard, W.M., Model atmospheres for X-ray bursting neutron stars, ApJ, 306, 170-182, 1986.

Madej J., Joss P.C., \& Róaska, A., Model Atmospheres and XRay Spectra of Bursting Neutron Stars: Hydrogen-Helium Comptonized Spectra, ApJ, 602, 904-912, 2004.

Majczyna A., Madej J., Joss P.C., \& Róaska, A., Model atmospheres and X-ray spectra of bursting neutron stars. II. Iron rich comptonized spectra, A\&A, 430, 643-654, 2005.

Markoff, S., \& Nowak, M.A., Constraining X-Ray Binary Jet Models via Reflection, ApJ, 609, 972-976, 2004.

Markoff, S., Nowak, M.A., \& Wilms, J., Going with the Flow: Can the Base of Jets Subsume the Role of Compact Accretion Disk Coronae?, ApJ, 635, 1203-1216, 2005

Markwardt, C.B., Altamirano, D., Swank, J.H., Strohmayer, T.E., Linares, M., \& Pereira, D., RXTE Detects 245 Hz X-ray Pulsations from IGR J17511-305, Atel, 2197, 2009.

Markwardt, C.B., Klein-Wolt, M., Swank, J.H., \& Wijnands, R. Burst Oscillations from IGR J17191-2821 Imply a $294 \mathrm{~Hz}$ Spin Frequency, Atel, 1068, 2007.

Markwardt, C.B., Swank, J.H., Strohmayer, T.E., in't Zand, J.J.M., \& Marshall, F.E., Discovery of a Second Millisecond Accreting Pulsar: XTE J1751-305, ApJ, 575, L21-L24, 2002.

Marshall, H.L., Constraints on the parameters of X-ray burster emission regions, ApJ, 260, 815-820, 1982.

Mastichiadis, A., \& Kylafis, N.D., Compton scattering in a converging fluid flow - Spherical near-critical accretion onto neutron stars, ApJ, 384, 136-142, 1992.

Maurer, I., \& Watts, A.L., Ignition latitude and the shape of Type I X-ray bursts, MNRAS, 383, 387-398, 2008.

McClintock, J. E., Canizares, C. R., \& Tarter, C. B., On the origin of 4640-4650 A emission in X-ray stars, ApJ, 198, 641-652, 1975.

Méndez, M., On the maximum amplitude and coherence of the kilohertz quasi-periodic oscillations in low-mass X-ray binaries, MNRAS, 371, 1925-1938, 2006.

Méndez, M., \& Belloni, T., Is there a link between the neutron-star spin and the frequency of the kilohertz quasi-periodic oscillations?, MNRAS, 381, 790-796, 2007.

Miller, J.M., Relativistic X-Ray Lines from the Inner Accretion Disks Around Black Holes, ARA\&A, 45, 441-479, 2007.
Miller, J.M., Fox, D.W., Di Matteo, T., et al., Relativistic Iron Emission and Disk Reflection in Galactic Microquasar XTE J1748-288, ApJ, 546, 1055-1067, 2001.

Miller, J.M., Reynolds, C.S., Fabian, A.C., \& Miniutti, G., \& Gallo, L.C., Stellar-Mass Black Hole Spin Constraints from Disk Reflection and Continuum Modeling, ApJ, 697, 900-912, 2009.

Miller, J.M., Wijnands, R., Méndez, M., XMM-Newton Spectroscopy of the Accretion-driven Millisecond X-Ray Pulsar XTE J1751-305 in Outburst, ApJ, 583, L99-L102, 2003.

Miller, M.C., \& Lamb, F.K., Motion of Accreting Matter near Luminous Slowly Rotating Relativistic Stars, ApJ, 470, 1033-1051, 1996.

Miller, M.C., \& Lamb, F.K., Bounds on the Compactness of Neutron Stars from Brightness Oscillations during X-Ray Bursts, ApJ, 499, L37-L40, 1998.

Miller, M.C., Lamb, F.K., \& Psaltis, Sonic-Point Model of Kilohertz Quasi-periodic Brightness Oscillations in Low-Mass X-Ray Binaries, ApJ, 508, 791-830, 1998.

Miller, M.C., Lamb, F.K., \& Psaltis, Constraints on the equation of state of neutron star matter from observations of kilohertz QPOs, Nuclear Physics B Proceedings Supplements, 69, 123-128, 1999.

Miniutti, G., Fabian, A.C., \& Anabuki, N., Suzaku Observations of the Hard X-Ray Variability of MCG -6-30-15: the Effects of Strong Gravity around a Kerr Black Hole, PASJ, 59, 315-325, 2007.

Misra, R., \& Kembhavi, A. K., Broadening of the Iron Emission Line in MCG -6-30-15 by Comptonization, ApJ, 499, 205-208, 1998.

Morsink, S.M., Leahy, D.A., Cadeau, C., \& Braga, J., The Oblate Schwarzschild Approximation for Light Curves of Rapidly Rotating Neutron Stars, ApJ, 663, 1244-1251, 2007.

Mukhopadhyay, B., Higher-Order Nonlinearity in Accretion Disks: Quasi-Periodic Oscillations of Black Hole and Neutron Star Sources and Their Spin, ApJ, 694, 387-395, 2009.

Muno, M.P., Chakrabarty, D., Galloway, D.K., \& Psaltis, D., The Frequency Stability of Millisecond Oscillations in Thermonuclear X-Ray Bursts, ApJ, 580, 1048-1059, 2002.

Muno, M.P., Özel, F., \& Chakrabarty, D., The Amplitude Evolution and Harmonic Content of Millisecond Oscillations in Thermonuclear X-Ray Bursts, ApJ, 581, 550-561, 2002.

Muñoz-Darias, T., Casares, J., \& Martínez-Pais, I. G., The "KCorrection" for Irradiated Emission Lines in LMXBs: Evidence for a Massive Neutron Star in X1822-371 (V691 CrA), ApJ, 635, 502-507, 2005.

Muñoz-Darias, T., Casares, J., O’Brien, K., Steeghs, D., MartínezPais, I. G., Cornelisse, R., Charles, P. A., Dynamical constraints on the neutron star mass in EXO 0748-676, MNRAS, 394, L136L140, 2009.

Narayan, R. \& Heyl, J.S., Thermonuclear Stability of Material Accreting onto a Neutron Star, ApJ, 599, 419-449, 2003.

Nath, N.R., Strohmayer, T.E., \& Swank, J.H., Bounds on Compactness for Low-Mass X-Ray Binary Neutron Stars from X-Ray Burst Oscillations, ApJ, 564, 353-360, 2002.

Nelson, L.A., \& Rappaport, S., Theoretical Considerations on the Properties of Accreting Millisecond Pulsars, ApJ, 598, 431-445, 2003.

Nelson, J., \& Sanders, G. H., The status of the Thirty Meter Telescope project, SPIE, 7012, 1-18, 2008.

Noble, M.S., \& Nowak, M.A., Beyond XSPEC: Toward Highly Configurable Astrophysical Analysis, PASP, 120, 821-837, 2008.

Orosz, J. A., \& Kuulkers, E., The optical light curves of Cygnus X-2 (V1341 Cyg) and the mass of its neutron star, MNRAS, 305, $132-142,1999$

Özel, F., Soft equations of state for neutron-star matter ruled out by EXO 0748 - 676, Nature, 441, 1115-1117, 2006.

Özel, F., What Makes an Accretion-Powered Millisecond Pulsar?, ApJ, 691, 1678-1683, 2009.

Özel, F., Güver, T., \& Psaltis, D., The Mass and Radius of the Neutron Star in EXO 1745-248, ApJ, 693, 1775-1779, 2009.

Özel, F., \& Psaltis, D., Spectral Lines from Rotating Neutron Stars, ApJ, 582, L31-L34, 2003.

Özel, F., \& Psaltis, D., Reconstructing the Neutron-Star Equation of State from Astrophysical Measurements, arXiv:0905.1959, 2009. 
Paczyński, B., A one-zone model for shell flashes on accreting compact stars, ApJ, 264, 282-295, 1983.

Pandel, D., Kaaret, P., \& Corbel, S., Relativistic Iron Line Emission from the Neutron Star Low-mass X-Ray Binary 4U 1636-536, ApJ, 688, 1288-1294, 2008.

Pandharipande, V.R., \& Smith, R.A., A model neutron solid with $\pi^{0}$ condensate, Nuclear Physics, A237, 507-532, 1975.

Papitto, A., Di Salvo, T., D'A', A., Iaria, R., Burderi, L., Riggio, A., Menna, M.T., \& Robba, N.R., XMM-Newton detects a relativistically broadened iron line in the spectrum of the ms X-ray pulsar SAX J1808.4-3658, A\&A, 493, L39-L43, 2009.

Parmar, A. N., White, N. E., Giommi, P., \& Gottwald, M., The discovery of 3.8 hour periodic intensity dips and eclipses from the transient low-mass X-ray binary EXO 0748-676, ApJ, 308, 199212, 1986.

Pechenick, K.R., Ftaclas, C., \& Cohen, J.M., Hot spots on neutron stars - The near-field gravitational lens, ApJ, 274, 846-857, 1983.

Piro, A.L., \& Bildsten, L., The Energy Dependence of Neutron Star Surface Modes and X-Ray Burst Oscillations, ApJ, 638, 968-973, 2006.

Poutanen, J., Modeling pulse profiles of accreting millisecond pulsars, AIP Conference Proceedings, 1068, 77-86, 2008.

Poutanen, J., \& Beloborodov, A.M., Pulse profiles of millisecond pulsars and their Fourier amplitudes, MNRAS, 373, 836-844, 2006.

Poutanen, J., \& Gierliński, M., On the nature of the X-ray emission from the accreting millisecond pulsar SAX J1808.4-3658, MNRAS, 343, 1301-1311, 2003.

Poutanen, J., \& Gierliński, M., Modelling the pulse profiles of accreting millisecond pulsars and X-ray bursters, Nuclear Physics B, 132, 640-643, 2004.

Psaltis, D., \& Chakrabarty, D., The Disk-Magnetosphere Interaction in the Accretion-powered Millisecond Pulsar SAX J1808.4-3658, ApJ, 521, 332-340, 1999.

Ray, P. S., Chakrabarty, D., Strohmayer, T. E., \& AXTAR Collaboration, The Advanced X-ray Timing Array (AXTAR), Bulletin of the American Astronomical Society, 41, 347-347, 2009.

Reeves, J.N., Porquet, D., \& Turner, T.J., An Extreme, Blueshifted Iron-Line Profile in the Narrow-Line Seyfert 1 PG 1402+261: An Edge-on Accretion Disk or Highly Ionized Absorption?, ApJ, 615, 150-155, 2004

Reis, R.C., Fabian, A.C., \& Young, A.J., Relativistically broadened iron line in the SUZAKU observation of the neutron star X-ray binary 4U 1705-44, MNRAS, in press, 2009.

Revnivtsev, M., Churazov, E., Gilfanov, M., \& Sunyaev, R., New class of low frequency QPOs: Signature of nuclear burning or accretion disk instabilities?, A\&A, 372, 138-144, 2001.

Reynolds, C.S., \& Nowak, M.A., Fluorescent iron lines as a probe of astrophysical black hole systems, Physics Reports, 377, 389-466, 2003.

Rutledge, R.E., Bildsten, L., Brown, E.F., Pavlov, G.G., \& Zavlin, V.E., Variable Thermal Emission from Aquila X-1 in Quiescence, ApJ, 577, 346-358, 2002.

Sahu, P.K., Basu, R., \& Datta, B., High-Density Matter in the Chiral Sigma Model, ApJ, 416, 267-275, 1993.

Schatz, H., et al., End Point of the rp Process on Accreting Neutron Stars, PRL, 86, 3471-3474, 2001.

Schatz, H., Bildsten, L., Cumming, A., \& Wiescher, M., The Rapid Proton Process Ashes from Stable Nuclear Burning on an Accreting Neutron Star, ApJ, 524, 1014-1029, 1999.

Schatz, H., \& Rehm, K. E., X-ray binaries, NuPhA, 777, 601-622, 2006.

Schwarzschild, M., \& Härm, R., Thermal Instability in NonDegenerate Stars, ApJ, 142, 855-867, 1965.

Shakura, N. I., \& Sunyaev, R. A., Black holes in binary systems. Observational appearance, A\&A, 24, 337-355, 1973.

Shaposhnikov, N., \& Titarchuk, L., On the Nature of the Flux Variability during an Expansion Stage of a Type I X-Ray Burst: Constraints on Neutron Star Parameters for 4U 1820-30, ApJ, 606, L57-L60, 2004.

Shaposhnikov, N., Titarchuk, L., \& Haberl, F., The Bursting Behavior of $4 \mathrm{U}$ 1728-34: The Parameters of a Neutron Star and the Geometry of a Neutron Star-Disk System, ApJ, 593, L35-L38, 2003.

Shaposhnikov, N., Titarchuk, L., \& Laurent, P., Discovery of Redskewed K $\alpha$ Iron Line in Cyg X-2 with Suzaku, ApJ, 699, 1223$1228,2009$.

Smale, A.P., A Type I Burst with Radius Expansion Observed from Cygnus X-2 with the Rossi X-Ray Timing Explorer, ApJ, 498, L141-L145, 1998.

Smale, A.P., A Second Intense Burst with Photospheric Radius Expansion from X2127+119 in M15, ApJ, 562, 957-962, 2001.

Spitkovsky, A., Levin, Y., \& Ushomirsky, G., Propagation of Thermonuclear Flames on Rapidly Rotating Neutron Stars: Extreme Weather during Type I X-Ray Bursts, ApJ, 566, 1018-1038, 2002. Spyromilio, J., Comerón, F., D’Odorico, S., Kissler-Patig, M., \& Gilmozzi, R., Progress on the European Extremely Large Telescope, The Messenger, 133, 2-8, 2008.

Srinivasan, G., Bhattacharya, D., Muslimov, A.G., \& Tsygan, A.J., A novel mechanism for the decay of neutron star magnetic fields, Current Science, 59, 31-38, 1990.

Steeghs, D., \& Casares, J., The Mass Donor of Scorpius X-1 Revealed, ApJ, 568, 273-278, 2002.

Stella, L., \& Vietri, M., Lense-Thirring Precession and Quasiperiodic Oscillations in Low-Mass X-Ray Binaries, ApJ, 492, L59L62, 1998.

Stella, L., \& Vietri, M., kHz Quasiperiodic Oscillations in Low-Mass X-Ray Binaries as Probes of General Relativity in the Strong-Field Regime, Physical Review Letters, 82, 17-20, 1999.

Strohmayer, T.E., \& Bildsten, L., New Views of Thermonuclear Bursts, in Compact Stellar X-ray Sources, Eds. W.H.G. Lewin and M. van der Klis, (Cambridge University Press: Cambridge), 39, 113-156, 2006.

Strohmayer, T.E., \& Brown, E.F., A Remarkable 3 Hour Thermonuclear Burst from 4U 1820-30, ApJ, 566, 1045-1059, 2002.

Strohmayer, T.E., Jahoda, K., Giles, A.B., \& Lee, U., Millisecond Pulsations from a Low-Mass X-Ray Binary in the Galactic Center Region, ApJ, 486, 355-362, 1997.

Strohmayer, T.E., Markwardt, C.B., Swank, J.H., \& in't Zand, J., X-Ray Bursts from the Accreting Millisecond Pulsar XTE J1814338, ApJ, 596, L67-L70, 2003.

Strohmayer, T.E., Zhang, W., \& Swank, J.H., 363 HZ Oscillations during the Rising Phase of Bursts from $4 \mathrm{U}$ 1728-34: Evidence for Rotational Modulation, ApJ, 487, L77-L80, 1997.

Strohmayer, T.E., Zhang, W., Swank, J.H., Smale, A., Titarchuk, L., Day, C., \& Lee, U., Millisecond X-Ray Variability from an Accreting Neutron Star System, ApJ, 469, L9-L12, 1996.

Strohmayer, T.E., Zhang, W., Swank, J.H., White, N.E., \& Lapidus, I., On the Amplitude of Burst Oscillations in 4U 1636-54: Evidence for Nuclear-powered Pulsars, ApJ, 498, L135-L139, 1998.

Syunyaev, R.A., \& Titarchuk, L.G., On the Spectra of X-Ray Bursters, SvAL, 12, 359-364, 1986.

Swank, J.H., Becker, R.H., Boldt, E.A., Holt, S.S., Pravdo, S.H., \& Serlemitsos, P.J., Spectral evolution of a long X-ray burst, ApJ, 212, L73-L76, 1977.

Sztajno, M., Fujimoto, M.Y., van Paradijs, J., Vacca, W.D., Lewin, W.H.G., Penninx, W., \& Trumper, J., Constraints on the massradius relation of the neutron star in $4 \mathrm{U} 1746-37 / \mathrm{NGC} 6441, \mathrm{MN}$ RAS, 226, 39-55, 1987.

Sztajno, M., van Paradijs, J., Lewin, W.H.G., Trumper, J., Stollman, G., Pietsch, W., \& van der Klis, M., Unusual X-ray burst profiles from 4U/MXB 1636-53, ApJ, 299, 487-495, 1985.

Taam, R.E., \& Picklum, R.E., Nuclear fusion and carbon flashes on neutron stars, ApJ, 224, 210-216, 1978.

Taam, R.E., Woosley, S.E., \& Lamb, D.Q., The Effect of Deep Hydrogen Burning in the Accreted Envelope of a Neutron Star on the Properties of X-Ray Bursts, ApJ, 459, 271-277, 1996.

Tanaka, Y., Nandra, K., Fabian, A.C., et al., Gravitationally redshifted emission implying an accretion disk and massive black hole in the active galaxy MCG-6-30-15, Nature, 375, 659-661, 1995.

Thampan, A.V., Bhattacharya, D., \& Datta, B., Implications of kHz quasi-periodic brightness oscillations in X-ray binaries for neutron star structure, MNRAS, 302, L69-L73, 1999. 
Thorsett, S.E., \& Chakrabarty, D., Neutron Star Mass Measurements. I. Radio Pulsars, ApJ, 512, 288-299, 1999.

Titarchuk, L., On the specta of X-ray bursters: Expansion and contraction stages, ApJ, 429, 340-355, 1994.

Titarchuk, L., Cui, W., \& Wood, K., Why Is It Difficult to Detect a Millisecond Pulsar in Neutron Star X-Ray Binaries?, ApJ, 576, L49-L52, 2002.

Titarchuk, L., Kazanas, D., \& Becker, P.A., Broad Redshifted Line as a Signature of Outflow, ApJ, 598, 411-418, 2003.

Titarchuk, L., Laurent, P., \& Shaposhnikov, N., On the Nonrelativistic Origin of Red-skewed Iron Lines in Cataclysmic Variable, Neutron Star, and Black Hole Sources, ApJ, 700, 1831-1846, 2009.

Titarchuk, L., \& Shaposhnikov, N., Three Type I X-Ray Bursts from Cygnus X-2: Application of Analytical Models for Neutron Star Mass and Radius Determination, ApJ, 570, L25-L28, 2002.

van den Heuvel, E. P. J., \& Bitzaraki, O., The magnetic field strength versus orbital period relation for binary radio pulsars with low-mass companions: evidence for neutron-star formation by accretion-induced collapse?, A\&A, 297, L41-L44, 1995.

van der Klis, M., Kilohertz Quasi-Periodic Oscillations in Low-Mass X-Ray Binaries, in Astronomical Time Series, Eds. D. Maoz, A. Sternberg and E.M. Leibowitz, (Kluwer), 218, 121, 1997.

van der Klis, M., Kilohertz Quasi-Periodic Oscillations in Low-Mass X-Ray Binaries, in The Many Faces of Neutron Stars, Eds. R. Buccheri, J. van Paradijs and M.A. Alpar, (Kluwer Academic Publishers: Boston), 515, 337, 1998.

van der Klis, M., Rapid X-ray Variability, in Compact Stellar X-ray Sources, Eds. W.H.G. Lewin and M. van der Klis, (Cambridge University Press: Cambridge), 39, 39-112, 2006.

van der Klis, M., Swank, J.H., \& Zhang, W., Discovery of Submillisecond Quasi-periodic Oscillations in the X-Ray Flux of Scorpius X-1, ApJ, 469, L1-L4, 1996.

van Kerkwijk, M.H., Properties of Neutron Stars, in Compact Stars: The Quest for New States of Dense Matter, Eds. D.K. Hong, C.H. Lee, H.K. Lee, D.-P. Min, T.-S. Park and M. Rho, (World Scientific Publishing Company: Singapore), 116, 2004.

van Paradijs, J., Average properties of X-ray burst sources, Nature, 274, 650-653, 1978

van Paradijs, J., Possible observational constraints on the massradius relation of neutron stars, ApJ, 234, 609-611, 1979

van Paradijs, J., On the maximum luminosity in X-ray bursts, A\&A, 101, 174-175, 1981.

van Paradijs, J., Some remarks on the spectra of X-ray bursts, A\&A, 107, 51-53, 1982.

van Paradijs, J., Dotani, T., Tanaka, Y., \& Tsuru, T., A very energetic X-ray burst from $4 \mathrm{U} 2129+11$ in M15, PASJ, 42, 633-660, 1990.

van Paradijs, J., \& Lewin, H.G., The interpretation of blackbody spectra and radii during X-ray bursts, A\&A, 157, L10-L12, 1986.

van Paradijs, J., \& Lewin, H.G., Constraints on the mass-radius relation for the neutron star in the X-ray burst source $4 \mathrm{U} / \mathrm{MXB}$ 1820-30 located in the globular cluster NGC 6624, A\&A, 172, L20-L22, 1987.

van Straaten, S., van der Klis, M., \& Wijnands, R., Relations Between Timing Features and Colors in Accreting Millisecond Pulsars, ApJ, 619, 455-482, 2005.

Villarreal, A.R., \& Strohmayer, T.E., Discovery of the Neutron Star Spin Frequency in EXO 0748-676, ApJ, 614, L121-L124, 2004.

Wade, R. A., \& Horne, K., The radial velocity curve and peculiar $\mathrm{TiO}$ distribution of the red secondary star in $\mathrm{Z}$ Chamaeleontis, ApJ, 324, 411-430, 1988.

Wallace, R.K., \& Woosley, S.E., Explosive hydrogen burning, ApJS, 43, 389-420, 1981.

Watts, A.L., Altamirano, D., \& Linares, M., Discovery of Burst Oscillations in the Intermittent Accretion-Powered Millisecond Pulsar HETE J1900.1-2455, ApJ, 698, L174-L177, 2009.

Watts, A.L., Altamirano, D., Markwardt, C.B., \& Strohmayer, T.E., Discovery of $245 \mathrm{~Hz}$ burst oscillations from the accreting millisecond pulsar IGR J17511-3057, Atel, 2199, 2009.

Watts, A.L., Patruno, A., \& van der Klis, M., Coherence of Burst Oscillations and Accretion-Powered Pulsations in the Accreting
Millisecond Pulsar XTE J1814-338, ApJ, 688, L37-L40, 2008.

Watts, A.L., \& Strohmayer, T.E., The energy dependence of burst oscillations from the accreting millisecond pulsar XTE J1814-338, MNRAS, 373, 769-780, 2006.

Watts, A.L., Strohmayer, T.E., \& Markwardt, C.B., Analysis of Variability in the Burst Oscillations of the Accreting Millisecond Pulsar XTE J1814-338, ApJ, 634, 547-564, 2005.

Webb, N.A., \& Barret, D., Constraining the Equation of State of Supranuclear Dense Matter from XMM-Newton Observations of Neutron Stars in Globular Clusters, ApJ, 671, 727-733, 2007.

Weinberg, N.N., Bildsten, L., Brown, E.F., Hydrodynamic Thermonuclear Runaways in Superbursts, ApJ, 650, L119-L122, 2006.

Weinberg, N.N., Bildsten, L., \& Schatz, H., Exposing the Nuclear Burning Ashes of Radius Expansion Type I X-Ray Bursts, ApJ, 639, 1018-1032, 2006.

Weinberg, N., Miller, M.C., \& Lamb, D.Q., Oscillation Waveforms and Amplitudes from Hot Spots on Neutron Stars, ApJ, 546, 10981106,2001

White, N. E., \& Swank, J. H., The discovery of 50 minute periodic absorption events from 4U 1915-05, ApJ, 253, L61-L66, 1982.

Wijnands, R., Accretion-driven millisecond X-ray pulsars, arXiv:astro-ph/0501264, 2005.

Wijnands, R., Guainazzi, M., van der Klis, M., \& Méndez, M., XMMNewton Observations of the Neutron Star X-Ray Transient KS 1731-260 in Quiescence, ApJ, 573, L45-L49, 2002.

Wijnands, R., Homan, J., Miller, J. M., \& Lewin, W. H. G., Monitoring Chandra Observations of the Quasi-persistent Neutron Star X-Ray Transient MXB 1659-29 in Quiescence: The Cooling Curve of the Heated Neutron Star Crust, ApJ, 606, L61-L64, 2004.

Wijnands, R., Strohmayer, T., \& Franco, L.M., Discovery of Nearly Coherent Oscillations with a Frequency of $567 \mathrm{HZ}$ during Type I X-Ray Bursts of the X-Ray Transient and Eclipsing Binary X1658298, ApJ, 549, L71-L75, 2001.

Wijnands, R., \& van der Klis, M., A millisecond pulsar in an X-ray binary system, Nature, 394, 344-346, 1998.

Wijnands, R., van der Klis, M., Homan, J., Chakrabarty, D., Markwardt, C.B., \& Morgan, E.H., Quasi-periodic X-ray brightness fluctuations in an accreting millisecond pulsar, Nature, 424, 4447, 2003.

Witten, E., Cosmic separation of phases, Physical Review D, 30, 272-285, 1984.

Woosley et al., Models for Type I X-Ray Bursts with Improved Nuclear Physics, ApJS, 151, 75-102, 2004.

Woosley, S.E., \& Taam, R.E., Gamma-ray bursts from thermonuclear explosions on neutron stars, Nature, 263, 101-103, 1976.

Yu, W. \& van der Klis, M., Kilohertz Quasi-periodic Oscillation Frequency Anticorrelated with Millihertz Quasi-periodic Oscillation Flux in 4U 1608-52, ApJ, 567, L67-L70, 2002.

Zhang, C., The MHD Alfven wave oscillation model of $\mathrm{kHz}$ Quasi Periodic Oscillations of Accreting X-ray binaries, A\&A, 423, 401404, 2004.

Zhang, C.M., The estimations of neutron star mass and radius by the $\mathrm{kHz}$ QPOs, Astron. Nachr., 330, 398-403, 2009 
Table 1: A few examples of neutron star LMXBs for which various parameter measurement methods have been attempted. Note that this list is not at all exhaustive.

\begin{tabular}{|c|c|c|}
\hline No. & Method (Section) ${ }^{a}$ & Source (Reference) ${ }^{b}$ \\
\hline 1 & $\begin{array}{l}\text { Burst Continuum Spectrum } \\
\text { Method (§ 3.1.3) }\end{array}$ & $\begin{array}{l}\text { EXO 0748-676 (Özel , 2006); } \\
\text { 4U 1746-37 (Sztajno et al. , 1987); } \\
\text { 4U 1636-536 (Fujimoto and Taam , 1986) }\end{array}$ \\
\hline 2 & $\begin{array}{l}\text { Burst Spectral Line Method } \\
(\S 3.1 .4)\end{array}$ & EXO 0748-676 (Cottam et al. , 2002; Özel , 2006) \\
\hline 3 & $\begin{array}{l}\text { Photospheric Radius Expansion } \\
\text { Burst Method }(\S 3.1 .5)\end{array}$ & $\begin{array}{l}\text { EXO 0748-676 (Özel , 2006); } \\
4 \text { U 1636-536 (Fujimoto and Taam , 1986); } \\
\text { X2127+119 (Smale , 1998); } \\
\text { Cygnus X-2 (Titarchuk and Shaposhnikov , 2002) }\end{array}$ \\
\hline 4 & Burst Oscillation Method ( $\S 3.1 .6)$ & $\begin{array}{l}\text { 4U 1636-536 (Nath et al. , 2002); } \\
\text { XTE J1814-338 (Bhattacharyya et al. , 2005) }\end{array}$ \\
\hline 5 & $\begin{array}{l}\text { Millihertz Quasi-periodic } \\
\text { Oscillation Method ( }(3.1 .7)\end{array}$ & Not attempted yet. \\
\hline 6 & $\begin{array}{l}\text { Accretion-powered Millisecond } \\
\text { Pulsation Method }(\S 3.2 .2)\end{array}$ & $\begin{array}{l}\text { SAX J1808.4-3658 (Poutanen and Gierliński , 2003; Leahy et al. , 2008); } \\
\text { XTE J1814-338 (Leahy et al. , 2009) }\end{array}$ \\
\hline 7 & $\begin{array}{l}\text { Kilohertz Quasi-periodic } \\
\text { Oscillation Method }(\S 3.3 .2)\end{array}$ & $\begin{array}{l}\text { 4U 1636-536 (Miller et al. , 1999); } \\
\text { Sco X-1 (Abramowicz et al. , 2003; Stella and Vietri , 1999); } \\
\text { GX 5-1 (Stella and Vietri , 1998); } \\
\text { GX 17+2 (Stella and Vietri , 1998); } \\
\text { SAX J1808.4-3658 (Zhang , 2009) }\end{array}$ \\
\hline 8 & $\begin{array}{l}\text { Broad Relativistic Iron Line } \\
\text { Method }(\S 3.4 .2)\end{array}$ & $\begin{array}{l}\text { Serpens X-1 (Bhattacharyya and Strohmayer, 2007b); } \\
4 \text { U 1820-30 (Cackett et al. , 2008a); } \\
\text { GX 349+2 (Cackett et al. , 2008a); } \\
\text { Cygnus X-2 (Cackett et al. , 2009b) }\end{array}$ \\
\hline 9 & $\begin{array}{l}\text { Quiescent Emission Method } \\
(\S 3.5 .2)\end{array}$ & $\begin{array}{l}\text { Sources in the globular clusters: omega Cen, M13, NGC 2808, NGC } 6304 \\
\text { (Webb and Barret , 2007; Guillot et al. , 2009) }\end{array}$ \\
\hline 10 & $\begin{array}{l}\text { Binary Orbital Motion Method } \\
(\S 3.6)\end{array}$ & $\begin{array}{l}\text { EXO 0748-676 (Muñoz-Darias et al. , 2009); } \\
\text { 4U 1822-37 (Muñoz-Darias et al. , 2005); } \\
\text { 4U 1254-69 (Barnes et al. , 2007) }\end{array}$ \\
\hline
\end{tabular}

${ }^{a}$ Neutron star parameter measurement methods and the sections of this review in which these methods have been described.

${ }^{b} \mathrm{~A}$ few example neutron star LMXBs for which the methods have been attempted; and the corresponding example references. 